TRANSACTIONS OF THE

AMERICAN MATHEMATICAL SOCIETY

Volume 351, Number 5, Pages 2101-2139

S 0002-9947(99)02469-1

Article electronically published on January 26, 1999

\title{
SYMPLECTIC GROUP LATTICES
}

\author{
RUDOLF SCHARLAU AND PHAM HUU TIEP
}

\begin{abstract}
Let $p$ be an odd prime. It is known that the symplectic group $S p_{2 n}(p)$ has two (algebraically conjugate) irreducible representations of degree $\left(p^{n}+1\right) / 2$ realized over $\mathbb{Q}(\sqrt{\epsilon p})$, where $\epsilon=(-1)^{(p-1) / 2}$. We study the integral lattices related to these representations for the case $p^{n} \equiv 1 \bmod 4$. (The case $p^{n} \equiv 3 \bmod 4$ has been considered in a previous paper.) We show that the class of invariant lattices contains either unimodular or $p$-modular lattices. These lattices are explicitly constructed and classified. Gram matrices of the lattices are given, using a discrete analogue of Maslov index.
\end{abstract}

\section{INTRODUCTION}

Let $p$ be an odd prime, and set $S_{n}=S p_{2 n}(p)$ for the symplectic group of degree $2 n$ over $\mathbb{F}_{p}$. Euclidean integral lattices in the space of the Weil representation of $S_{n}$ have been investigated by several authors (see for instance [BaV], [Dum], [Gow], [Gro], [Tiep 1], [Tiep 2]). The Weil representation $\mathcal{W}$ of $S_{n}$ is a complex representation of degree $p^{n}$ that can be obtained from the action of $S_{n}$ on the extraspecial group $p_{+}^{1+2 n}$ (as the outer automorphism group). See, for example, [Isa], [Sei], or [Ward 1] for a more general approach. $\mathcal{W}$ is a sum of two irreducible representations of degrees $\left(p^{n}-1\right) / 2$ and $\left(p^{n}+1\right) / 2$. ( These two characters seem to have been first investigated in [BRW].) One of these representations, which we shall denote by $\mathcal{W}_{1}$, is faithful and has even degree, and the kernel of the other representation, $\mathcal{W}_{2}$, is the center $Z=C_{2}$ of $S_{n}$. Following [Gow], we shall refer to $\mathcal{W}_{1}$ and $\mathcal{W}_{2}$ as Weil representations. Weil representations have been characterized in several ways in [TZa 1], [TZa 2].

Set $\epsilon=(-1)^{(p-1) / 2}$ and suppose that $\left(\operatorname{dim} \mathcal{W}_{i}, \epsilon\right) \neq\left(\frac{p^{n}-1}{2}, 1\right)$. It is shown in [Gro] that, under this assumption, the character $\psi_{i}$ of the representation $\mathcal{W}_{i}$ generates the field $\mathbb{Q}(\sqrt{\epsilon p})$ over the rational field $\mathbb{Q}$, and has Schur index 1 over $\mathbb{Q}$. Hence, there exist an extension $G_{n}$ of $S_{n}$ and an absolutely irreducible $\mathbb{Q} G_{n^{-}}$ module $V$ affording the $S_{n}$-character $\psi_{i}+\bar{\psi}_{i}$, where the bar denotes the algebraic conjugation of the field $\mathbb{Q}(\sqrt{\epsilon p})$. The group $G_{n}$ can be chosen as a homomorphic

Received by the editors December 10, 1996.

1991 Mathematics Subject Classification. Primary 20C10, 20C15, 20C20, 11E12, 11H31, 94B05.

Key words and phrases. Integral lattice, unimodular lattice, $p$-modular lattice, finite symplectic group, Weil representation, Maslov index, linear code, self-dual code.

Part of this work was done during the second author's stay at the Department of Mathematics, Israel Institute of Technology. He is grateful to Professor D. Chillag and his colleagues at the Technion for stimulating conversations and their generous hospitality. His work was also supported in part by the DFG.

(C)1999 American Mathematical Society 
image of the conformal symplectic group

$$
\begin{aligned}
G_{n}=C S p_{2 n}(p)=\{\varphi \in G L(W) \mid & \exists \kappa \in \mathbb{F}_{p}^{\bullet}, \forall u, v \in W, \\
& \langle\varphi(u), \varphi(v)\rangle=\kappa\langle u, v\rangle\},
\end{aligned}
$$

where $W$ denotes a natural $2 n$-dimensional $S_{n}$-module over $\mathbb{F}_{p}$, with the symplectic form $\langle\cdot, \cdot\rangle$. In what follows we shall be concerned with the following two homomorphic images of $G_{n}$ : the factor-group $G_{n}^{+}$of $G_{n}$ by its center $C_{p-1}$ (consisting of the scalar matrices $\lambda E_{2 n}, \lambda \in \mathbb{F}_{p}^{\bullet}$ ), and $G_{n}^{-}$, the factor-group of $G_{n}$ by the central group $C_{(p-1) / 2}$ (consisting of scalar matrices $\lambda E_{2 n}, \lambda \in \mathbb{F}_{p}^{\bullet 2}$ ). Throughout the paper, $C_{m}$ denotes the cyclic group of order $m$, and $E_{m}$ denotes the identity matrix of order $m$ (over any field).

The lattices for the Weil representations of degree $\psi(1)=\left(p^{n}-1\right) / 2$ have been investigated in [Gow] and [Gro]. Recall that in this case $p \equiv 3 \bmod 4$, according to our general assumption; see [Tiep 2] for the excluded case. If $n$ is even, then every $\mathbb{Z} S_{n}$-lattice in $V$ is even unimodular. If $n$ is odd, $V$ contains $p$-modular invariant lattices. Recall that an integral lattice $\Lambda$ is said to be $p$-modular (or modular of level $p$ ) if the lattices ${ }^{p} \Lambda^{\#}$ (the dual lattice $\Lambda^{\#}$ rescaled by the scalar $p$ ) and $\Lambda$ are isometric. $p$-modular lattices have been introduced and investigated in [CoS 1] and [Que]. In either of these cases, the corresponding representations are globally irreducible in the sense of Gross [Gro]. Some of the corresponding lattices have been realized as sublattices of the Mordell-Weil lattices of certain elliptic curves (cf. [Dum] and [Gro]).

The Weil representations of degree $\psi(1)=\left(p^{n}+1\right) / 2$ are the subject of our present work, begun in $[\mathrm{SchT}]$ and continued in this paper. Here the corresponding representation cannot be globally irreducible anymore; namely, $\psi \bmod 2=1_{S}+\eta$ for some $\eta \in \operatorname{IBr}_{2}(S)$. In [SchT], the case $p^{n} \equiv 3 \bmod 4$ has been treated. The existence of unimodular $\mathbb{Z} G$-lattices in $V$ has been established, where $G=G_{n}^{-} \simeq S_{n} \cdot C_{2}$. All $\mathbb{Z} G$-lattices contained in $V$ have been classified.

In this paper, we are concerned with the case $p^{n} \equiv 1 \bmod 4$. Then $\mathcal{W}_{2}$ viewed over $\mathbb{Q}$ is in fact a faithful representation of $P S p_{2 n}(p)$ of degree $p^{n}+1$. Moreover, if $p \equiv 3 \bmod 4$, this representation can be extended (in a unique way) to a rational representation of $G_{n}^{+}$. If $p \equiv 1 \bmod 4$, it can be extended to a rational representation for each of the two groups $G_{n}^{+}$and $G_{n}^{-}$(cf. Proposition 2.3). The reason is that when $p \equiv 1 \bmod 4$ the two groups $C_{2} \times G_{n}^{+}$and $G_{n}^{-}$are isoclinic to each other. For more detail on isoclinic groups see [Atlas] and [Tiep 2], Lemma 2.11. When $p \equiv 3 \bmod 4$, it follows from this lemma that the rational representation of $S_{n}$ of degree $p^{n}+1$ is extendible to a rational faithful representation of $G_{n}^{+}$if $n$ is even, and of $G_{n}^{-}$if $n$ is odd, but not for its isoclinic variant.

From now on we keep the following notation: $S_{n}=S p_{2 n}(p), G_{n}=C S p_{2 n}(p)$, $Z \cong C_{p-1}$ the center of $G_{n}^{+}, G_{n}^{+}=G_{n} / Z, G_{n}^{-}=G_{n} / Z^{2}, \theta$ a fixed generating element of $\mathbb{F}_{p}^{\bullet}$. Clearly, $G_{n}$ is generated by $S_{n}$ and an element $\vartheta_{n}$ with matrix $\operatorname{diag}\left(E_{n}, \theta E_{n}\right)$ in a fixed symplectic basis $\left(e_{1}, \ldots, e_{n}, f_{1}, \ldots, f_{n}\right)$ of the natural $S_{n^{-}}$ module $W=W_{n}=\mathbb{F}_{p}^{2 n}$ (that is, a basis in which the symplectic form $\langle\cdot, \cdot\rangle$ is given as follows: $\left.\left\langle e_{i}, e_{j}\right\rangle=0,\left\langle f_{i}, f_{j}\right\rangle=0,\left\langle e_{i}, f_{j}\right\rangle=\delta_{i, j}\right) . V=V_{n}$ denotes an irreducible $\mathbb{Q} G_{n}$-module with character $\chi$ such that $\left.\chi\right|_{S_{n}}=\psi+\bar{\psi}$. Furthermore, either $\operatorname{Ker} \chi=Z$ (and then $V_{n}$ is a faithful $G_{n}^{+}$-module), or $p \equiv 1 \bmod 4$ and $\operatorname{Ker} \chi=Z^{2}$ (and then $V_{n}$ is a faithful $G_{n}^{-}$-module). Under these assumptions $\chi$ exists and is unique by Proposition 2.3. It is clear that there exists a unique (up to scalar) $G_{n}$-invariant positive definite symmetric bilinear form $(\cdot, \cdot)$ on $V_{n}$. 
Our first main result is the following theorem which includes Theorem 1.1 of [SchT]:

Theorem 1.1. Let $p$ be an odd prime. If $p \equiv 3 \bmod 4$, then suppose in addition that $n$ is odd. Then $V_{n}$ contains $G_{n}^{-}$-invariant odd unimodular Euclidean lattices (of rank $\left.p^{n}+1\right)$. If $n>1$, these lattices have no roots.

Actually, we provide an explicit construction of a $G_{n}^{-}$-stable odd unimodular lattice $\Delta=\Delta(p, n)$ contained in $V_{n}$ (cf. Theorem 3.9 and Corollary 5.4) for $n$ odd, and a $G_{n}^{-}$-stable odd unimodular lattice $\Delta^{-}(p, n)$ for the case where $n$ is even and $p \equiv 1 \bmod 4$ (cf. Corollary 5.9). In the case $p \equiv 3 \bmod 4$ this is just the construction exposed in $[\mathrm{SchT}]$. The cases $p^{n}=27$ and $p^{n}=25$ have been considered by R. Bacher and B. B. Venkov $[\mathrm{BaV}]$, and G. Nebe, respectively. The corresponding lattices have minimum 3. In general, Theorem 7.1 yields $\min \Delta(p, n) \geq(p+1) / 2$ for all $n \geq 3$.

Our next results are concerned with $p$-modular lattices.

Theorem 1.2. Let $p$ be any odd prime. If $p \equiv 3 \bmod 4$, then suppose in addition that $n$ is even. Then $V_{n}$ contains $G_{n}^{+}$-invariant $p$-modular Euclidean lattices (of $\left.\operatorname{rank} p^{n}+1\right)$.

Again, we provide an explicit construction of a $G_{n}^{+}$-stable $p$-modular lattice $\Delta=$ $\Delta^{-}(p, n)$ if $n$ is odd and $p \equiv 1 \bmod 4$ (cf. Corollary 5.10), respectively $\Delta=\Delta(p, n)$ if $n$ is even (cf. Theorem 4.4 and Corollary 5.6). This result generalizes Theorem (V.2) of [NPl] dealing with the case $n=1$ and $p \equiv 1 \bmod 4$. The case $n=2$ and $p=5$ has been considered by Nebe; the corresponding 5 -modular lattice $(\Delta(5,2)$ in our notation) has minimum 5 . As before, Theorem 7.1 yields $\min \Delta(p, n) \geq(p+1) / 2$ for all $n \geq 2$.

Gram matrices of the lattices $\Delta(p, n), \Delta^{-}(p, n)$ are given in $\S 5$ using a discrete analogue of Maslov index. In a few words, our explicit constructions can be described as follows. First we start with the case $n$ is odd and the group $G_{n}^{-}$. Using Lagrangians, an idea going back to $[\mathrm{BaV}]$ (cf. §3) and Maslov index (cf. §5), we explicitly construct the unimodular lattices $\Delta(p, n)$ (for any odd prime $p$ ). Then descending from $n+1$ to $n$ (cf. $\S 4$ ), we obtain the $p$-modular lattices $\Delta(p, n)$ which are stable under $G_{n}^{+}$, for any even $n$ and any odd prime $p$. Finally, let $p \equiv 1 \bmod 4$. Then the isoclinism between $C_{2} \times G_{n}^{+}$and $G_{n}^{-}$and Proposition 2.4 allow us to construct the lattices $\Delta^{-}(p, n)$, for any $n$.

We wish to point out that $V$ cannot contain simultaneously invariant unimodular and $p$-modular lattices. Namely, the unimodular lattices are acted on faithfully by $G_{n}^{-}$, and the $p$-modular ones by $G_{n}^{+}$. Moreover, the invariant unimodular $(p$ modular) lattices are essentially unique (if they exist). More precisely, the classification of $G_{n}$-invariant lattices in $V_{n}$ is provided by the following theorem. Given a lattice $\Gamma$, let $\Gamma^{\#}$ denote the dual lattice and $\Gamma^{0}$ denote the sublattice consisting of all vectors of even norm in $\Gamma$; furthermore, $\Gamma^{1}=\Gamma \cap 2\left(\Gamma^{0}\right)^{\#}$. Two integral lattices $(\Gamma,(\cdot, \cdot))$ and $\left(\Gamma^{\prime},(\cdot, \cdot)^{\prime}\right)$ are called similar if there exist a surjective homomorphism $\phi: \Gamma \rightarrow \Gamma^{\prime}$ and a scalar $\lambda \in \mathbb{Q}$ such that $(\phi(u), \phi(v))^{\prime}=\lambda(u, v)$ for any $u, v \in \Gamma$.

Theorem 1.3. Let $p$ be any odd prime and $n$ any integer. Suppose that $G_{n}$ acts irreducibly on an integral lattice $\Gamma$ of rank $p^{n}+1$, with kernel $K$. If $p^{n}=3$ or $p^{n}=p \equiv 1 \bmod 6$, then suppose in addition that $S_{n}$ acts reducibly on $\Gamma \otimes \mathbb{C}$. Then one of the following holds. 
(i) $G_{n} / K$ is equal to $G_{n}^{-}$for odd $n$, and $G_{n}^{+}$for even $n$. Furthermore, $\Gamma$ is similar to one of the lattices $\Delta, \Delta^{0}, \Delta^{1}$, where $\Delta=\Delta(p, n)$.

(ii) $p \equiv 1 \bmod 4$, and $G_{n} / K$ is equal to $G_{n}^{+}$for odd $n$, and $G_{n}^{-}$for even $n$. Furthermore, $\Gamma$ is similar to one of the lattices $\Delta, \Delta^{0}, \Delta^{1}$, where $\Delta=\Delta^{-}(p, n)$.

The $G L_{2}(p)$-invariant $(p+1)$-dimensional lattices which are not covered by Theorem 1.3 (here $p=3$ or $p \equiv 1 \bmod 6$ ) have been investigated in [NPl], Theorem (V.4).

The full automorphism groups of all $G_{n}$-invariant lattices $\Lambda$ in $V_{n}$ have been determined in [Tiep 1]. In particular, if $n>1$, then either $\operatorname{Aut}(\Lambda) \in\left\{C_{2} \times G_{n}^{+}, G_{n}^{-}\right\}$, or $p=3$ and $\operatorname{Aut}(\Lambda)=\left(C_{6} \cdot P S p_{2 n}(3)\right) \cdot C_{2}$.

\section{IMPLICIT PROOFS}

We recall the notations $S_{n}, \psi$ of degree $\left(p^{n}+1\right) / 2, G_{n}, Z, G_{n}^{+}, G_{n}^{-}$. We start with the following simple observation:

Lemma 2.1. Let $p$ be any odd prime and $n$ any integer. Suppose $\chi$ is an irreducible complex character of $G_{n}$ of degree $p^{n}+1$ with the following properties:

(i) $\chi$ restricted to $S_{n}$ is equal to $\psi+\bar{\psi}$;

(ii) $\chi$ is rational-valued.

Then one of the following holds.

(a) $\chi$ is a faithful character, say $\chi^{+}$, of $G_{n}^{+}$; furthermore, $n$ is even if $p \equiv$ $3 \bmod 4$

(b) $\chi$ is a faithful character, say $\chi^{-}$, of $G_{n}^{-}$; furthermore, $n$ is odd if $p \equiv$ $3 \bmod 4$.

Proof. Let $K=\operatorname{Ker} \chi$. Schur's Lemma and (ii) imply that $Z /(Z \cap K)$ is a cyclic group of order at most 2. In particular, either $K=Z$, or $K=Z^{2}$. Observe that $G_{n}$ permutes the two characters $\psi$ and $\bar{\psi}$ of $S_{n}$ nontrivially. Denote $\bar{G}=G_{n} / K$, $\bar{S}=S_{n} /\left(S_{n} \cap K\right)$.

First consider the case $K=Z$. Since $K \subseteq \operatorname{Ker} \psi$, the degree $\psi(1)$ is odd, i.e. $p^{n} \equiv 1 \bmod 4$. Here we have $\bar{G}=G_{n}^{+}, \bar{S}=P S p_{2 n}(p)$, and $\bar{G}=\bar{S} \cdot C_{2}$. Clearly, the desired character $\chi$ is now uniquely determined: $\chi=\operatorname{Ind}_{\bar{S}} \bar{G}(\psi)$.

Next let $K=Z^{2}$. If $p \equiv 1 \bmod 4$, then $\bar{G}=(\bar{S} \times Z / K) \cdot C_{2}$. If $p \equiv 3 \bmod 4$, then in view of (i) $n$ must be odd, and $\bar{G}=\bar{S} \cdot C_{2}$. Now the desired character $\chi$ exists and is unique: $\chi=\operatorname{Ind}_{\bar{S}}^{\bar{G}}(\psi)$ if $p \equiv 3 \bmod 4$, and $\chi=\operatorname{Ind}_{\bar{S} \times Z / K}^{\bar{G}}(\widetilde{\psi})$ if $p \equiv 1 \bmod 4$ (where $\widetilde{\psi}$ is equal to $\psi$ on $\bar{S}$ and to $-\psi(1)$ on the unique nontrivial element of $Z / K)$. Clearly, $\mathbb{Q}(\chi)=\mathbb{Q}$.

As we have mentioned above, $C_{2} \times G_{n}^{+}$and $G_{n}^{-}$are isoclinic to each other if $p \equiv 1 \bmod 4 . \quad$ In this case, $\operatorname{ind}(\psi)=1$ (cf. [Gro], Corollary 13.7); hence by Lemma 2.11 of [Tiep 2] ind $\left(\chi^{+}\right)=\operatorname{ind}\left(\chi^{-}\right)=1$. If $p \equiv 3 \bmod 4$ and $n$ is odd, then $\operatorname{ind}(\psi)=0$, and ind $\left(\chi^{-}\right)=1$ (see [SchT] or Proposition 2.3 below). Hence by Lemma 2.11 of [Tiep 2], the corresponding character of degree $p^{n}+1$ of the isoclinic variant of $G_{n}^{-}$(which now is not isomorphic to $C_{2} \times G_{n}^{+}$) has Schur-Frobenius indicator -1 and so cannot be written over $\mathbb{R}$. Similarly, if $p \equiv 3 \bmod 4$, then $\operatorname{ind}(\psi)=0$, ind $\left(\chi^{+}\right)=1$, but the corresponding character of degree $p^{n}+1$ of the isoclinic variant of $C_{2} \times G_{n}^{+}$(which is no longer $G_{n}^{-}$) cannot be written over $\mathbb{R}$.

The next proposition is an analogue of [SchT], Lemma 5.1. 
Proposition 2.2. Let $p$ be an odd prime and $n$ any integer. Let $r$ be any prime and let $\chi$ be as in Lemma 2.1. Then the following assertions hold.

(i) The reduction $\chi \bmod r$ is irreducible if $r \neq 2, p$.

(ii) $\chi \bmod 2=2 \cdot 1_{G_{n}}+\beta$ for a certain $\beta \in \operatorname{IBr}_{2}\left(G_{n}\right)$. Furthermore, $\beta$ is of symplectic type, if $p \equiv 1 \bmod 4$.

(iii) $\chi \bmod p=\eta_{1}+\eta_{2}$, where $\eta_{1}, \eta_{2} \in \operatorname{IBr}_{p}\left(G_{n}\right)$ are distinct characters which can be written over $\mathbb{F}_{p}$. Furthermore, for $k=1,2 \eta_{k}$ is not self-dual if $\chi=\chi^{-}$, and $\eta_{k}$ is of type + if $\chi=\chi^{+}$.

Proof. The case $p^{n} \equiv 3 \bmod 4$ has already been handled in [SchT] (cf. the proof of Theorem 1.1 and Lemma 5.1 therein). Hence in what follows we suppose that $p^{n} \equiv 1 \bmod 4$.

1) It is well-known (see e.g. [Gow], [Gro]) that $\psi \bmod r \in \operatorname{IBr}_{r}\left(S_{n}\right)$ for any odd prime $r$. Furthermore, $\psi \bmod 2=1_{S_{n}}+\alpha$ for some $\alpha \in \operatorname{IBr}_{2}\left(S_{n}\right)$. If $x$ is a regular unipotent element of $S_{n}$, then $\psi(x)=\left(1 \pm p^{n-1} \sqrt{\epsilon p}\right) / 2$. Furthermore, $\vartheta_{n}$ interchanges the $S_{n}$-conjugacy classes of $x$ and some power of $x^{s}$ and $\psi\left(x^{s}\right)=$ $\left(1 \mp p^{n-1} \sqrt{\epsilon p}\right) / 2$. Therefore, $\chi \bmod r \in \operatorname{IBr}_{r}\left(G_{n}\right)$ for any prime $r, r \neq 2, p$. On the other hand, $\chi \bmod 2=2 \cdot 1_{G_{n}}+\beta$ for some $\beta \in \operatorname{IBr}_{2}\left(G_{n}\right)$. If $p \equiv 1 \bmod 4$, then the fact that $\alpha$ is of symplectic type has been established in [GoW]. From this it follows by [Tiep 2], Lemma 2.4 that $\beta$ is of symplectic type.

2) Consider the reduction $\chi \bmod p$. Recall that $\left.\chi\right|_{S_{n}}=\psi+\bar{\psi}$. It is shown in [Gro] that $\psi \bmod p=\bar{\psi} \bmod p=\eta$ is obtained by restricting the irreducible algebraic representation of $S p_{2 n}\left(\overline{\mathbb{F}_{p}}\right)$ with highest weight $\frac{p-1}{2} \omega_{n}$ to $S_{n}$. Furthermore, due to Lemma 2.6 [Tiep 3], $\eta$ is invariant under the action of the distinguished element $\vartheta_{n}$. Therefore, $G_{n}$ has just two irreducible Brauer characters $\eta_{1}, \eta_{2}$ with $\left.\eta_{k}\right|_{S_{n}}=\eta$ and $\eta_{1}+\eta_{2}=0$ on $G_{n} \backslash S_{n}$. In this case, $\chi \bmod p=\eta_{1}+\eta_{2}$, since $\chi=0$ on $G_{n} \backslash S_{n}$.

3) We can embed $S_{n}$ into $T=S p_{2 n}\left(p^{2}\right)$ in the following way. In a natural $2 n$-dimensional $\mathbb{F}_{p^{2}}$-module $\widetilde{W}$ of $T$ consider a symplectic basis

$$
\left(e_{1}, \ldots, e_{n}, f_{1}, \ldots, f_{n}\right) \text {. }
$$

In this basis we can set $W=\left\langle e_{1}, \ldots, e_{n}, f_{1}, \ldots, f_{n}\right\rangle_{\mathbb{F}_{p}}, J=\operatorname{diag}\left(\varsigma^{-1} E_{n}, \varsigma E_{n}\right)$. Here $\varsigma \in \mathbb{F}_{p^{2}}$ is chosen with order $2(p-1)$ such that $\theta=\varsigma^{2}$. Now we set $S_{n}=$ $T \cap \operatorname{End}(W) \simeq S p_{2 n}(p), H=\left\langle S_{n}, J\right\rangle$. Then $J^{2} \in S_{n}$ and $J$ normalizes $S_{n}$; therefore $H \simeq S_{n} \cdot C_{2}$. Furthermore, $H / Z\left(S_{n}\right) \simeq G_{n}^{+}$.

Assume $p \equiv 1 \bmod 4$. Factoring the embedding $S \hookrightarrow S p_{2 n}\left(\overline{\mathbb{F}_{p}}\right)$ through $T=$ $S p_{2 n}\left(p^{2}\right)$, one sees that $\eta$ is extended to two absolutely irreducible Brauer characters $\mu_{1}, \mu_{2}$ of $H$. We calculate the value of $\mu_{1}, \mu_{2}$ at the element $J$. If one denotes $\sigma=\exp \left(\frac{\pi i}{p-1}\right)$, then $\mu_{1}(J)=\sum_{u \in I_{n}^{+}} \sigma^{|u|}$, since $n(p-1) / 2$ is even in our case. Here

$$
\begin{gathered}
I_{n}^{+}=\left\{u=\left(u_{1}, \ldots, u_{n}\right)\left|u_{j} \in \mathbb{Z},\right| u_{j} \mid \leq(p-1) / 2, \sum_{j=1}^{n} u_{j} \equiv 0 \bmod 2\right\}, \\
I_{n}^{-}=\left\{u=\left(u_{1}, \ldots, u_{n}\right)\left|u_{j} \in \mathbb{Z},\right| u_{j} \mid \leq(p-1) / 2, \sum_{j=1}^{n} u_{j} \equiv 1 \bmod 2\right\}, \\
S_{n}^{+}=\sum_{u \in I_{n}^{+}} \sigma^{|u|}, S_{n}^{-}=\sum_{u \in I_{n}^{-}} \sigma^{|u|},
\end{gathered}
$$


and $|u|=\sum_{j} u_{j}$ for $u \in I_{n}^{ \pm}$. Denote also $\tau=\cot \left(\frac{\pi}{2(p-1)}\right)$. Then we have

$$
\begin{gathered}
S_{1}^{+}=\frac{\tau-\tau^{-1}}{2}, S_{1}^{-}=\frac{\tau+\tau^{-1}}{2}, \\
S_{n+1}^{+}=S_{n}^{+} S_{1}^{+}+S_{n}^{-} S_{1}^{-}, S_{n+1}^{-}=S_{n}^{+} S_{1}^{-}+S_{n}^{-} S_{1}^{+} .
\end{gathered}
$$

From this it follows that

$$
S_{n}^{+}=\frac{\tau^{n}+(-\tau)^{-n}}{2}, S_{n}^{-}=\frac{\tau^{n}-(-\tau)^{-n}}{2} .
$$

In particular, $\mu_{1}(J)=\left(\tau^{n}+(-\tau)^{-n}\right) / 2$. Since $p \geq 5, \tau>1$, and so $\mu_{1}(J)$ is a positive real number. Moreover, the Frobenius endomorphism $\sigma \mapsto \sigma^{p}$ sends $\sigma$ to $-\sigma, i=\sigma^{(p-1) / 2}$ to $i, \mu_{1}(J)$ to $\mu_{1}(J)=-\mu_{2}(J)$. We have shown that $\mu_{k}^{(p)}=\mu_{k}=\overline{\mu_{k}}$ for $k=1,2$.

If $p \equiv 3 \bmod 4$, then $n$ is even. Under the above notation, the computation in the proof of $[\mathrm{SchT}]$, Lemma 5.1 shows that $\mu_{1}(J)=\left(\tau^{n}+\tau^{-n}\right) / 2$. In particular, $\mu_{1}(J)$ is again a positive real number. Moreover, the Frobenius endomorphism $\sigma \mapsto \sigma^{p}$ sends $\sigma$ to $-\sigma, i=\sigma^{(p-1) / 2}$ to $-i, \mu_{1}(J)$ to $\mu_{1}(J)=-\mu_{2}(J)$. Therefore, $\mu_{k}^{(p)}=\mu_{k}=\overline{\mu_{k}}$ for $k=1,2$.

4) Here we consider the case $\chi=\chi^{+}$. Since Ker $\mu_{k}=Z\left(S_{n}\right)$ and $H / Z\left(S_{n}\right)$ $\simeq G_{n}^{+}$, we have $\eta_{k}=\mu_{k}, k=1,2$. Thus $\eta_{k}$ can be written over $\mathbb{F}_{p}$. Furthermore, since $\eta_{k}$ is real-valued and $\left.\eta_{k}\right|_{S_{n}}=\eta$ is of quadratic type, $\eta_{k}$ itself is of quadratic type.

5) Next let $\chi=\chi^{-}$. Then $p \equiv 1 \bmod 4$. Consider a representation $\Phi$ : $H \rightarrow G L_{\left(p^{n}+1\right) / 2}\left(\overline{\mathbb{F}_{p}}\right)$ with Brauer character $\mu_{1}$. Put $\omega=\varsigma^{(p-1) / 2}$, and set $\widetilde{G}=$ $\left\{ \pm \Phi(g), \pm \omega \Phi(h) \mid g \in S_{n}, h \in H \backslash S_{n}\right\}$. Since $\omega^{2}=-1, \widetilde{G}$ is a group. We claim that $\widetilde{G} \simeq \bar{G}=G_{n} / K=G_{n}^{-}$, where $K=\operatorname{Ker} \chi \simeq Z^{2}$. For the proof, we first observe that $\widetilde{G}$ is generated by the subgroup $G^{\prime}=\left\{ \pm \Phi(g) \mid g \in S_{n}\right\}$ and the element $\omega \Phi(J)$. Observe that the representation $\Phi$ is not faithful: its kernel is equal to the center $Z\left(S_{n}\right)$ of $S_{n}$. But the factor-group $H / Z\left(S_{n}\right) \simeq P S p_{2 n}(p) \cdot C_{2}$ has trivial center; therefore $\Phi(H)$ also has trivial center. In particular, $G^{\prime} \simeq S_{n} / Z\left(S_{n}\right) \times C_{2}$. The subgroup $C_{2}$ here is generated by $\mathbf{j}$, the multiplication by -1 (on the representation space of $\Phi)$; hence we can identify $\mathbf{j}$ with the central element $\theta E_{2 n}$ in $\bar{G}$. Now one has:

$$
\begin{gathered}
\vartheta_{n}^{2}=\operatorname{diag}\left(E_{n}, \theta^{2} E_{n}\right)=\theta E_{2 n} \cdot \operatorname{diag}\left(\theta^{-1} E_{n}, \theta E_{n}\right)=\mathbf{j} J^{2}, \\
(\omega \Phi(J))^{2}=-\Phi\left(J^{2}\right)=\mathbf{j} \Phi\left(J^{2}\right) .
\end{gathered}
$$

Modulo $Z\left(S_{n}\right)=\operatorname{Ker}\left(\left.\Phi\right|_{S_{n}}\right)$ one can identify the two elements $\vartheta_{n}^{2}$ and $(\omega \Phi(J))^{2}$. Furthermore, the actions of $\omega \Phi(J)$ and of $\vartheta_{n}$ on $S_{n}$ via conjugation are obviously the same. This means that $\widetilde{G} \simeq \bar{G}$.

The isomorphism $\bar{G} \simeq \widetilde{G}$ gives us a representation $\Psi: G_{n} \rightarrow G L_{\left(p^{n}+1\right) / 2}\left(\overline{\mathbb{F}_{p}}\right)$ with kernel $K$. One may suppose that this representation affords the Brauer character $\eta_{1}$. Then $\eta_{1}\left(\vartheta_{n}\right)=\sqrt{-1} \mu_{1}(J)$. The computations in item 3$)$ show that $\eta_{1}\left(\vartheta_{n}\right)$ is purely imaginary, and that the Frobenius endomorphism ${ }^{(p)}$ leaves $\eta_{1}\left(\vartheta_{n}\right)$ fixed. Consequently, for $k=1,2$ the Brauer character $\eta_{k}$ can be realized over $\mathbb{F}_{p}$ but it is not real.

Proposition 2.3. Let $p$ be any odd prime and $n$ any integer. Let $\chi$ be as in Lemma 2.1. Then $\chi$ is afforded by a $\mathbb{Q} G_{n}$-module (of dimension $p^{n}+1$ ). 
Proof. 1) First we give an argument settling the case where $n$ is odd and $\chi=\chi^{-}$. Let $q=p^{n}$. Then we can identify $W$ with $\mathbb{F}_{q}^{2}$, and endow $W$ with the symplectic form $\langle u, v\rangle=\operatorname{tr}(a d-b c)$, where $u=(a, b), v=(c, d), a, b, c, d \in \mathbb{F}_{q}$, and tr stands for the trace form $\operatorname{tr}: \mathbb{F}_{q} \rightarrow \mathbb{F}_{p}$. Make the group

$$
R=\left\{\varphi \in H=G L_{2}(q) \mid \operatorname{det} \varphi \in \mathbb{F}_{p}^{\bullet}\right\} \simeq S L_{2}(q) \cdot C_{p-1}
$$

act on $W$ in a natural way. Clearly, this action embeds $R$ in $G_{n}=C S p(W)$. Let $T$ denote the central subgroup $\left\{\operatorname{diag}(\lambda, \lambda) \mid \lambda \in \mathbb{F}_{q}^{\bullet 2}\right\} \simeq C_{(q-1) / 2}$ of $H$. Then the assumption that $n$ is odd implies that $T \cap R=K$, where $K=\operatorname{Ker} \chi=C_{(p-1) / 2}$, and $R T=H$. Hence, $\left.\chi\right|_{R}$ can be viewed as a faithful character of $R / K=R /(T \cap R) \simeq$ $H / T$ and so as a character, say $\rho$, of $H$ (with kernel $T$ ). Beside that, the restriction of $\chi$ to the subgroup $R^{\prime}=S L_{2}(q)$ is the sum of two irreducible Weil characters of degree $(q+1) / 2$ of $R^{\prime}$. Inspecting the character table of $H$ (cf. [DiM]) we see that $\rho=\operatorname{Ind}_{B}^{H}(\mu)$, where

$$
B=\left\{\left(\begin{array}{cc}
a & b \\
0 & d
\end{array}\right) \mid a, d \in \mathbb{F}_{q}^{\bullet}, c \in \mathbb{F}_{q}\right\}
$$

is a Borel subgroup of $H$, and the linear character $\mu$ sends $\left(\begin{array}{cc}a & b \\ 0 & d\end{array}\right)$ to $\delta(a), \delta$ the quadratic character of $\mathbb{F}_{q}$. In particular, $\rho$ is rational and absolutely irreducible. The same is true for $\left.\chi\right|_{R}$. Now a standard lemma (see for instance [KoT], Lemma 8.3.1) says that $\chi$ is also rational.

2) Now we give another argument handling the case $p^{n} \equiv 1 \bmod 4$. The equality $\mathbb{Q}(\chi)=\mathbb{Q}$ implies by the Brauer-Speiser theorem that the Schur index $m_{\mathbb{Q}}(\chi)$ is either 1 or 2 . If $m_{\mathbb{Q}}(\chi)=1$, we are done. Assuming $m_{\mathbb{Q}}(\chi)=2$, we get an irreducible $\mathbb{Q} G_{n}$-module $V$ with character $2 \chi$. Clearly, the commuting algebra $\mathbb{K}=\operatorname{End}_{G_{n}}(V)$ is a quaternion algebra over $\mathbb{Q}$. If a prime $r$ is ramified in $\mathbb{K}$, then there exists a Brauer character $\mu$ such that $\chi \bmod r=\mu+\mu^{(r)}$. By Proposition 2.2 , this cannot occur for any prime $r$. Thus $\mathbb{K}$ is unramified at any prime $r$, and by Hasse's principle we get a contradiction.

Having established Proposition 2.3, we are given a $\mathbb{Q} G$-module $V=V_{n}$ with character $\chi$ such that $\left.\chi\right|_{S_{n}}=\psi+\bar{\psi}$, where $(G, \chi)=\left(G_{n}^{+}, \chi^{+}\right)$or $\left(G_{n}^{-}, \chi^{-}\right)$. We shall maintain this notation in what follows.

Proof of Theorem 1.1. Proposition 2.2 shows that the pair $(G, \chi)=\left(G_{n}^{-}, \chi^{-}\right)$satisfies the conditions (i), (ii) of Proposition 2.4 from [SchT]. Below (cf. (7) and Corollary 4.3) we shall see that condition (iii) is also fulfilled: $\operatorname{det} V=\mathbb{Q}^{\bullet 2}$. Applying that proposition, we obtain a $G_{n}^{-}$-invariant unimodular lattice $\Gamma$. Standard arguments show that $\Gamma$ is odd, and $\min \Gamma \geq 3$ if $n>1$.

The link between lattices invariant under isoclinic groups is indicated in the following statement. We make use of the following observation: for any prime $p \equiv 1 \bmod 4$, there exist $a, b \in \mathbb{N}$ such that $a^{2}-p b^{2}=-1$ (cf. [Coh], pp. 105, 106). Henceforth we fix such a pair $\mathbf{a}, \mathbf{b}$.

Proposition 2.4. Let $p \equiv 1 \bmod 4$ be a prime. Let $G^{+} \simeq H \cdot C_{2}$ and $G^{-}=$ $\left(H \cdot C_{2}\right)^{-}$be isoclinic groups, where $H$ is a finite group with center of order 2. Suppose $V$ is an absolutely irreducible $\mathbb{Q} G^{+}$-module with character $\chi^{+}$, and $\mathbb{Q}(\psi)=$ $\mathbb{Q}(\sqrt{p})$ where $\left.\chi^{+}\right|_{H}=\psi+\bar{\psi}$. Then the following assertions hold. 
(i) $V$ can be viewed as an absolutely irreducible $\mathbb{Q} G^{-}$-module. In particular, for each sign $\varepsilon \in\{+,-\}, V$ has a unique (up to scalar) $G^{\varepsilon}$-invariant scalar product $(\cdot, \cdot)^{\varepsilon}$.

(ii) $V$ has an endomorphism $\sigma$ with the following properties: $\sigma$ centralizes the group $H, \sigma^{2}=p \cdot \mathrm{id}_{V}, \sigma$ is a self-adjoint similarity of norm $p$ w.r.t. both scalar products $(\cdot, \cdot)^{ \pm}$, and

$$
g \sigma g^{-1}=-\sigma \text { for } g \in G^{ \pm} \backslash H .
$$

(iii) $V$ contains $\sigma$-stable lattices which are invariant under both groups $G^{+}$and $G^{-}$.

(iv) Let $\Lambda$ be a lattice as in (iii), and denote by $\Lambda^{\varepsilon}, \varepsilon \in\{+,-\}$, its dual lattice with respect to $(\cdot, \cdot)^{\varepsilon}$. After suitably rescaling one of $(\cdot, \cdot)^{\varepsilon}$, we have the equality

$$
\Lambda^{-}=\sigma^{-1}\left(\Lambda^{+}\right)
$$

On this scale, $\Lambda$ is unimodular w.r.t. $(\cdot, \cdot)^{+}$if and only if it is p-modular w.r.t. $(\cdot, \cdot)^{-}$. Similarly, $\Lambda$ is $p$-modular w.r.t. $(\cdot, \cdot)^{+}$if and only if it is unimodular w.r.t. $\frac{1}{p}(\cdot, \cdot)^{-}$.

Proof. The equality $\left.\chi\right|_{H}=\psi+\bar{\psi}$ implies that the commuting algebra

$$
\mathbb{K}=\operatorname{End}_{H}(V)=\left\{\varphi \in \operatorname{End}_{\mathbb{Q}}(V) \mid \forall h \in H, \varphi \cdot h=h \cdot \varphi\right\}
$$

is isomorphic to the field $\mathbb{Q}(\psi)=\mathbb{Q}(\sqrt{p})$. Denoting by $\sigma$ the (unique up to $\operatorname{sign}$ ) element $\sigma \in \mathbb{K}$ with $\sigma^{2}=p \cdot \mathrm{id}_{V}$, we have to show that it satisfies all other properties stated. We begin with (1). Fix an element $g \in G^{+} \backslash H$. For $h \in H$ and $\lambda \in \mathbb{K}$ it is readily checked that $h \cdot g \lambda g^{-1}=g \lambda g^{-1} \cdot h$; in other words, $g \lambda g^{-1} \in \mathbb{K}$. Thus, conjugation by $g$ induces an automorphism of $\mathbb{K}$. If $\lambda$ is fixed by this automorphism, then $\lambda$ centralizes $H$ and $g$, and thus $G^{+}$, that is, $\lambda \in \mathbb{Q}$. Thus, $\lambda \mapsto g \lambda g^{-1}$ is the unique nontrivial field automorphism of $\mathbb{K}$. Property $(1)$ is a special case of this $(\sigma$ corresponds to $\sqrt{p}$ ).

If $\Gamma$ is any $G^{+}$-invariant lattice, then $\Gamma+\sigma(\Gamma)$ is clearly $\sigma$-invariant, and by (1) still $G^{+}$-invariant, which proves (iii) in the "+"-case. The scalar product $(\sigma(x), \sigma(y)), x, y \in V$, is also $G^{+}$-invariant and therefore of the form $(\sigma(x), \sigma(y))=$ $c(x, y)$ for some $c \in \mathbb{R}$. From $\sigma^{2}=p \cdot \mathrm{id}_{V}$ it follows that $c=p$. The self-adjointness

$$
(\sigma(x), y)^{+}=(x, \sigma(y))^{+}
$$

now is a formal consequence, and part (i) is proved for $(\cdot, \cdot)^{+}$.

Recall that we have fixed positive integers $\mathbf{a}, \mathbf{b}$ with $\mathbf{a}^{2}-p \mathbf{b}^{2}=-1$. Denote by $\rho \mapsto \bar{\rho}$ the non-trivial automorphism of $\mathbb{K}$, that is, $g \rho=\bar{\rho} g$ for any $g \in G^{+} \backslash H$ (see above), and by $\mu$ the particular element

$$
\mu=\mathbf{a}+\mathbf{b} \sigma \in \mathbb{K} .
$$

Since $\mu \circ \bar{\mu}=\bar{\mu} \circ \mu=-\mathrm{id}_{V}, \bar{\mu}=\mathbf{a}-\mathbf{b} \sigma$, this element induces in fact an automorphism of any $G^{+}$-stable lattice. If $g \in G^{+} \backslash H$ and $g^{\prime}:=g \mu$, then $g^{\prime 2}=g \mu g \mu=g^{2} \bar{\mu} \mu=$ $-g^{2}$. Thus $\left\langle H, g^{\prime}\right\rangle \cong G^{-}$. Observe that the particular representation of $G^{-}$thus constructed is obviously absolutely irreducible, and an $H$-invariant $\sigma$-stable lattice is $G^{+}$-invariant if and only if it is $G^{-}$-invariant.

For a given choice of $(\cdot, \cdot)$, consider the bilinear form

$$
(x, y)^{-}:=(x, \sigma \mu(y))^{+}
$$

which is clearly $H$-invariant. Since $\sigma \mu$ is self-adjoint w.r.t. $(\cdot, \cdot)^{+}$, this bilinear form is symmetric. From the fact that $\sigma \mu$ is a totally positive element in $\mathbb{K}$ it easily 
follows that $(\cdot, \cdot)^{-}$is also positive definite. The following computation shows that $(\cdot, \cdot)^{-}$is invariant under $g^{\prime}=g \mu$ and thus under all of $G^{-}$:

$$
\begin{aligned}
\left(g^{\prime} x, g^{\prime} y\right)^{-} & =(g \mu(x),(\sigma \mu) g \mu(y))^{+} \\
& =(g \mu(x), g \overline{\sigma \mu} \mu(y))^{+} \\
& =(\mu(x), \overline{\sigma \mu} \mu(y))^{+} \\
& =(\mu(x), \sigma(y))^{+} \\
& =(x, \mu \sigma(y))=(x, y)^{-}
\end{aligned}
$$

(since $\bar{\mu} \mu=-\mathrm{id}_{V}$ ).

For the dual lattices, we clearly have

$$
\Lambda^{-}=(\sigma \mu)^{-1} \Lambda^{+}=\sigma^{-1} \mu^{-1} \Lambda^{+}=\sigma^{-1} \Lambda^{+} .
$$

If $\Lambda$ is unimodular w.r.t. $(\cdot, \cdot)^{+}$, then $\Lambda^{+}=\Lambda$; therefore $\Lambda^{-}=\sigma^{-1}(\Lambda)$, i.e. $\sigma$ is the desired similarity between $\Lambda$ and $\Lambda^{-}$. Conversely, if $\Lambda$ is $p$-modular w.r.t. $(\cdot, \cdot)^{-}$, then

$$
\begin{gathered}
p^{\operatorname{rank} \Lambda / 2}=\left(\Lambda^{-}: \Lambda\right)=\left(\sigma^{-1}\left(\Lambda^{+}\right): \Lambda\right) \\
=\left(\Lambda^{+}: \sigma(\Lambda)\right)=\left(\Lambda^{+}: \Lambda\right)(\Lambda: \sigma(\Lambda))=p^{\operatorname{rank} \Lambda / 2}\left(\Lambda^{+}: \Lambda\right),
\end{gathered}
$$

yielding $\Lambda^{+}=\Lambda$. The last assertion follows from the previous one by considering $(x, y)^{--}:=(x, \sigma(-\bar{\mu})(y))^{-}$.

Clearly, Proposition 2.4 applies to $\left\{G^{+}, G^{-}\right\}=\left\{C_{2} \times G_{n}^{+}, G_{n}^{-}\right\}$and the module $V=V_{n}$, if $p \equiv 1 \bmod 4$. Also, the endomorphism $\sigma$ is uniquely determined up to sign. Therefore, in what follows we can speak about $\sigma$-stable lattices in $V$.

We shall need the following supplement to Proposition 2.4:

Lemma 2.5. Keep all the notation of Proposition 2.4. Suppose that, as $\mathbb{F}_{p} H$ module, $U:=\Lambda / p \Lambda$ is the direct sum of two copies $M, M^{\prime}$ of an absolutely irreducible $\mathbb{F}_{p} H$-module. Then the $\mathbb{F}_{p} G^{+}$-module $U$ is indecomposable if and only if the $\mathbb{F}_{p} G^{-}$-module $U$ is indecomposable.

Proof. By our assumption, in a suitably chosen basis of $U=M \oplus M^{\prime}$ the commuting algebra $K:=\operatorname{End}_{H}(U)$ consists of matrices of the form $\left(\begin{array}{cc}a E_{n} & b E_{n} \\ c E_{n} & d E_{n}\end{array}\right)$, where $a, b, c, d \in \mathbb{F}_{p}$ and $n=\operatorname{dim} M$. Clearly, the endomorphism $\sigma$ (cf. Proposition 2.4 (ii)) belongs to $K$, and without loss of generality one may suppose that $\sigma=$ $\left(\begin{array}{cc}0 & E_{n} \\ 0 & 0\end{array}\right)$, because $\sigma^{2}=0$ on $U$. Recall that $G^{+}=\langle H, g\rangle$ and $G^{-}=\left\langle H, g^{\prime}\right\rangle$ with $g^{\prime}=g(\mathbf{a}+\mathbf{b} \sigma)$. Since $g \sigma=-\sigma g, g$ has the matrix $\left(\begin{array}{cc}A & B \\ 0 & -A\end{array}\right)$, and $g^{\prime}$ has the matrix $\left(\begin{array}{cc}\mathbf{a} A & \mathbf{b} A+\mathbf{a} B \\ 0 & -\mathbf{a} A\end{array}\right)$. Denoting $L^{+}=\operatorname{End}_{G^{+}}(U)$, we see that $L^{+}=C_{K}(g)$. In particular, if $f=\left(\begin{array}{cc}x E_{n} & y E_{n} \\ z E_{n} & t E_{n}\end{array}\right)$ belongs to $L^{+}$, then either $f=x E_{2 n}$, or $x \neq t$ and $B=\frac{2 y}{x-t} A$. Now observe that: the $\mathbb{F}_{p} G^{+}$-module $U$ is decomposable if and only if $L^{+}$contains two nonzero idempotents $f, g$ such that $f g=g f=0$ if and only if $B \in\langle A\rangle_{\mathbb{F}_{p}}$. The same applies to the $\mathbb{F}_{p} G^{-}$-module $U$. But

$$
B \in\langle A\rangle_{\mathbb{F}_{p}} \Longleftrightarrow \mathbf{b} A+\mathbf{a} B \in\langle\mathbf{a} A\rangle_{\mathbb{F}_{p}}
$$

hence our statement follows. 
Corollary 2.6. Let $p \equiv 1 \bmod 4$ and $n$ any integer. Then $V_{n}$ contains $\sigma$-stable $G_{n}^{-}$-invariant odd unimodular lattices.

Proof. By Proposition 2.4, $V_{n}$ contains $\sigma$-stable $G_{n}^{-}$-invariant lattices. Choose such a lattice $\Gamma$ with minimal possible determinant, and suppose that $\operatorname{det} \Gamma>1$. Clearly, the symmetry of $\sigma$ implies that the dual lattice $\Gamma^{\#}$ is $\sigma$-stable. In particular, taking the sum $\Gamma+m \Gamma^{\#}, m \in \mathbb{Z}$, produces again a $\sigma$-stable lattice. Hence Lemma 2.1 [SchT] holds inside the class of $\sigma$-stable lattices. Now the arguments in the proof of [SchT], Proposition 2.4, show that $A=\Gamma^{\#} / \Gamma=\left(C_{2}\right)^{2}$. Consider the form $q: A \rightarrow \frac{1}{2} \mathbb{Z} / \mathbb{Z}, q(v+\Gamma)=(v, v)+\mathbb{Z}$. Clearly, $\sigma$ acts on $A$ as an endomorphism of order 1 or 2 , and $\sigma$ preserves $q$. If $\sigma=1$ on $A$, then we are done by Lemma 2.2 [SchT]. If $\sigma \neq 1$ on $A$, then $\sigma$ has a unique nonzero fixed vector $\bar{v}$ in $A$. This vector is obviously fixed by $G_{n}^{-}$, and one can check that $q(\bar{v})=0$. Thus $\langle\Gamma, v\rangle$ is a $\sigma$-stable $G_{n}^{-}$-invariant unimodular lattice, contrary to the choice of $\Gamma$.

A $\sigma$-stable $G_{n}^{-}$-invariant odd unimodular lattice will be explicitly constructed in Theorem 3.9 (for odd $n$ ) and Corollary 5.9 (for even $n$ and $p \equiv 1 \bmod 4$ ).

Proof of Theorem 1.2 for the case $p \equiv 1 \bmod 4$. By Corollary 2.6, $V_{n}$ contains a $\sigma$ stable $G_{n}^{-}$-invariant unimodular lattice $\nabla$. Applying Proposition 2.4, we obtain a $G_{n}^{+}$-invariant scalar product on $\nabla$ that converts it into a $p$-modular lattice which is acted on by $G_{n}^{+}$.

Remark 2.7. Let $n=1$ and $p \equiv 1 \bmod 4$. Then Proposition 2.3 and its proof tell us that the group $G_{1}^{-}=G L_{2}(p) / C_{(p-1) / 2}$ has a (unique) faithful, absolutely irreducible, rational representation of degree $p+1$, which is monomial. On the other hand, $G_{1}^{+}=P G L_{2}(p)$ has a (unique) faithful, absolutely irreducible, rational representation of degree $p+1$, which is non-monomial. These observations were mentioned in [NPl], Lemma (V.3) and its proof. Theorem (V.2) of [NPl] exposes a $G_{1}^{+}$-invariant lattice of rank $p+1$ and determinant $p^{(p+1) / 2}$, called $M_{p+1,2}$. Observe that $M_{p+1,2}$ is obtained from the $G_{1}^{-}$-invariant lattice with Gram matrix $E_{p+1}$ by means of the procedure indicated in Proposition 2.4. Hence by this proposition $M_{p+1,2}$ is $p$-modular.

An explicit construction of $G_{n}^{-}$-invariant odd unimodular lattices is exposed in Theorem 3.9 for any odd $n$. Combined with the procedure indicated in Proposition 2.4 , this yields an explicit construction of $G_{n}^{+}$-invariant $p$-modular lattices $\Delta^{-}(p, n)$ for any odd $n$ (cf. Corollary 5.10), which generalizes Theorem (V.2) of [NPl].

The rest of the section is devoted to proving Theorem 1.2 for the case $p \equiv$ $3 \bmod 4$. Denote $\theta=\sqrt{-p}, \pi=(1+\theta) / 2, \mathbb{K}=\mathbb{Q}(\theta), \mathfrak{o}=\langle 1, \pi\rangle_{\mathbb{Z}}$ the maximal order in $\mathbb{K}$. By Proposition 5.2 [SchT], whose proof does not use the oddness of $n$, $V=V_{n}$ has an endomorphism $\sigma$ such that

$$
\begin{gathered}
\sigma^{2}(v)=-p v,(\sigma(u), v)=-(u, \sigma(v)),(\sigma(u), \sigma(v))=p(u, v), \\
s \sigma s^{-1}=\sigma, g \sigma g^{-1}=-\sigma
\end{gathered}
$$

for any $u, v \in V, s \in \bar{S}_{n}=P S p_{2 n}(p), g \in G_{n}^{+} \backslash \bar{S}_{n}$. Following the proof of that proposition, it is not difficult to show that $V$ contains a $G_{n}^{+}$-invariant lattice $\Lambda$, which is stable under the endomorphism $(1+\sigma) / 2$. For $u, v \in V$ we set

$$
u \circ v=\frac{(u, v)}{2}+\theta \frac{(u, \sigma(v))}{2 p} \in \mathbb{K} .
$$


Using (2) it is straightforward to check that $v \circ u=\overline{u \circ v}, u \circ \sigma(v)=-\theta(u \circ v)$, $s u \circ s v=u \circ v, g u \circ g v=v \circ u$, and

$$
(u, v)=\operatorname{Tr}(u \circ v),
$$

where $s \in \bar{S}_{n}, g \in G_{n}^{+} \backslash \bar{S}_{n}$, and $\operatorname{Tr}$ denotes the trace of $\mathbb{K}$ over $\mathbb{Q}$. Thus, if we set $\theta \cdot v=\sigma(v)$, then $V$ is a $\mathbb{K}$-space of dimension $\left(p^{n}+1\right) / 2$, with $\bar{S}_{n}$-invariant positive definite Hermitian scalar product $u \circ v$. Multiplying $(\cdot, \cdot)$ by a suitable scalar, for instance by $2 p$, we can ensure that $\Lambda \circ \Lambda \subseteq \mathfrak{o}$. Thus $\Lambda$ is a $G_{n}^{+}$-invariant integral o-lattice, that is contained in its Hermitian dual,

$$
\Gamma^{\perp}=\{u \in V \mid u \circ \Gamma \subseteq \mathfrak{o}\} .
$$

The property (4) by the way characterizes the Hermitian form uniquely, that is, implies (3). Clearly, both $\Lambda^{\perp}$ and $\Lambda^{\#}$, the Euclidean dual, are stable under $\mathfrak{o}$ and $G_{n}^{+}$. Using (3), one readily checks that

$$
\Lambda^{\perp}=\theta \Lambda^{\#}
$$

(This is actually well known from (4), since $(\theta)$ is the different of $\mathfrak{o}$ over $\mathbb{Z}$.) We shall use this formula frequently in what follows. We shall also need the following two simple statements.

Lemma 2.8. Let $p \equiv 3 \bmod 4$ as above, and let $G$ be a finite group. Suppose that $\Gamma$ is a $G$-invariant integral Hermitian o-lattice such that $\Gamma \supseteq 2 \Gamma^{\perp}$. Then the $\mathbb{F}_{2} G$-module $\Gamma^{\perp} / \Gamma$ supports a non-degenerate $G$-invariant alternating form, namely $b(u, v)=\operatorname{Tr}(2 u \circ v) \bmod 2$ for any $u, v \in \Gamma^{\perp}$. In particular, the index $\left(\Gamma^{\perp}: \Gamma\right)$ differs from 2 for any integral hermitian o-lattice $\Gamma$.

Proof. Since $\Gamma \supseteq 2 \Gamma^{\perp}, 2 u \in \Gamma$ and so $2 u \circ v \in \mathfrak{o}$ and $\operatorname{Tr}(2 u \circ v) \in \mathbb{Z}$ for all $u, v \in \Gamma^{\perp}$. If $v \in \Gamma$, then $2 u \circ v \in 2 \mathfrak{o}$ and $\operatorname{Tr}(2 u \circ v) \in 2 \mathbb{Z}$. Thus $b$ is well defined. Clearly, it is $\mathbb{F}_{2}$-bilinear and $G$-invariant. If $u \in \Gamma^{\perp}$, then $2 u \circ u \in \mathbb{R} \cap \mathfrak{o}=\mathbb{Z}$, yielding $b(u, u)=0$, i.e. $b$ is alternating. Finally, assume that $v \in \Gamma^{\perp}$ such that $\operatorname{Tr}(u \circ v) \in \mathbb{Z}$ for any $u \in \Gamma^{\perp}$. Then $v \in\left(\Gamma^{\perp}\right)^{\#}=\theta^{-1} \Gamma^{\perp \perp}=\theta^{-1} \Gamma$ and $\theta^{-1} \Gamma \cap(1 / 2) \Gamma=\Gamma$. In other words, $b$ is non-degenerate.

Lemma 2.9. Let $\Gamma$ be an $\bar{S}_{n}$-invariant o-lattice in $V$. Suppose that the index $\left(\Gamma^{\perp}: \Gamma\right)$ is divisible by $p$. Then in fact $\Gamma \subseteq p \Gamma^{\perp}$.

Proof. Consider the $\mathbb{F}_{p} \bar{S}_{n}$-module $U=\Gamma / \theta \Gamma$. Here we are identifying $\mathfrak{o} / \theta \mathfrak{o}$ with $\mathbb{F}_{p}$ (and $\pi$ with $1 / 2$ ). First we show that $\Gamma \subseteq \theta \Gamma^{\perp}$. We know that $U$ is a simple $\mathbb{F}_{p} S_{n}$-module with character $\psi \bmod p$. Furthermore, $U^{\prime}=\left(\Gamma \cap \theta \Gamma^{\perp}\right) / \theta \Gamma$ is an $S_{n^{-}}$ submodule of $U$. Suppose that $U^{\prime}=0$. Then $\Gamma \cap \theta \Gamma^{\perp}=\theta \Gamma$. As $\left(\Gamma^{\perp}: \Gamma\right)$ is divisible by $p$, one can find a vector $v \in \theta \Gamma^{\perp} \backslash \theta \Gamma$ such that $p v \in \theta \Gamma$. Then

$$
\theta v \in p \Gamma^{\perp} \cap \Gamma \subseteq \theta \Gamma^{\perp} \cap \Gamma=\theta \Gamma,
$$

i.e. $v \in \Gamma$. Hence, $v \in \Gamma \cap \theta \Gamma^{\perp}=\theta \Gamma$, contradicting the choice of $v$. Therefore, $U^{\prime} \neq 0$, which implies that $U^{\prime}=U, \Gamma \subseteq \theta \Gamma^{\perp}$.

Now we can define on $U$ an $S_{n}$-invariant form:

$$
f(\bar{x}, \bar{y})=\frac{1}{\theta} x \circ y \bmod \theta \mathfrak{o},
$$

where $\bar{x}=x+\theta \Gamma, \bar{y}=y+\theta \Gamma$. Clearly, $f$ is well defined and bilinear. But $f$ is alternating: $f(\bar{x}, \bar{x})=0$ because $x \circ x \in \mathbb{R} \cap \theta \mathfrak{o}=\theta^{2} \mathbb{Z}$ for any $x \in \Gamma$. Suppose the kernel of $f$ is zero. Then $U$ carries a non-degenerate alternating bilinear form 
(namely $f$ ) and so $\psi \bmod p$ is of symplectic type, contrary to Proposition 2.2 (iii). We have shown that the kernel of $f$ is nonzero. Since $U$ is irreducible, $f$ is zero, i.e. $\Gamma \subseteq p \Gamma^{\perp}$.

Now we choose a $G_{n}^{+}$-invariant o-lattice $\Lambda$ lying in $V$ such that $\operatorname{det} \Lambda=\left(\Lambda^{\#}: \Lambda\right)$ is minimal. We also suppose that $\Lambda$ is not integral for any rescaled Hermitian form $\frac{1}{\lambda} u \circ v$ with $\lambda \in \mathbb{R}$ and $\lambda>1$. (If such a $\lambda$ exists, we simply divide the Hermitian scalar product to $\lambda$ and get an invariant Hermitian lattice with strictly smaller determinant.)

First we observe that $\operatorname{det} \Lambda$ cannot be divisible by any odd prime $r \neq p$. Suppose the contrary. Then consider the form $(\bar{x}, \bar{y})_{r}=(x, y) \bmod r$ on $\Lambda / r \Lambda$, where $\bar{x}=$ $x+r \Lambda, \bar{y}=y+r \Lambda$. As $r$ divides $\operatorname{det} \Lambda$, this $G_{n}^{+}$-invariant symmetric bilinear form is degenerate, and so its kernel $\left(\Lambda \cap r \Lambda^{\#}\right) / r \Lambda$ is nonzero. By Proposition 2.2 (i) this means simply that $\Lambda \subseteq r \Lambda^{\#}$. Then for any $u, v \in \Lambda$ one has $(u, v),(u, \pi v) \in r \mathbb{Z}$. Denote $u \circ v=a+\pi b$ for some $a, b \in \mathbb{Z}$. Then $(u, v)=2 a+b$ and $(u, \pi v)=$ $a-b(p-1) / 2$ belong to $r \mathbb{Z}$. This implies $a, b \in r \mathbb{Z}$. In particular, $u \circ v \in r o$ for any $u, v \in \Lambda$. Thus, one can divide the form $u \circ v$ by $r$, a contradiction.

We have seen that det $\Lambda$ can be divisible only by the primes 2 and $p$. Furthermore, if $p$ divides $\left(\Lambda^{\perp}: \Lambda\right)$, then by Lemma 2.9 one can divide the form $u \circ v$ by $p$, a contradiction. Therefore, there exists a non-negative integer $k$ such that $\left(\Lambda^{\perp}: \Lambda\right)=$ $2^{k}$. In this case,

$$
\operatorname{det} \Lambda=\left(\Lambda^{\#}: \Lambda^{\perp}\right)\left(\Lambda^{\perp}: \Lambda\right)=2^{k} p^{N}
$$

where $N=\left(p^{n}+1\right) / 2$.

It is obvious that $\Lambda \supseteq 2^{k} \Lambda^{\perp}$. Suppose that $\Lambda \nsupseteq 2 \Lambda^{\perp}$. Let $l$ denote the minimal integer such that $\Lambda \supseteq 2^{l} \Lambda^{\perp}$. Then $l \geq 2$. Set $\Gamma=\Lambda+2^{l-1} \Lambda^{\perp}$. One readily checks that $\Gamma$ is a $G_{n}^{+}$-invariant o-lattice with $\operatorname{det} \Gamma$ strictly smaller than $\operatorname{det} \Lambda$, contradicting the choice of $\Lambda$. Hence, $\Lambda \supseteq 2 \Lambda^{\perp}$. This implies that $\Lambda \supseteq 2 p \Lambda^{\#}$, i.e. the discriminant group $\Lambda^{\#} / \Lambda$ has exponent $2 p$ (and order $2^{k} p^{N}$ ).

The inclusion $\Lambda \supseteq 2 p \Lambda^{\#}$ also implies $k \leq 2 N$. Setting $\Gamma=\sqrt{2} \Lambda^{\perp}$ (which is equivalent to considering $\Lambda^{\perp}$ and multiplying the form $u \circ v$ by 2), we have

$$
\Gamma \circ \Gamma=\sqrt{2} \Lambda^{\perp} \circ \sqrt{2} \Lambda^{\perp}=2 \Lambda^{\perp} \circ \Lambda^{\perp} \subseteq \Lambda \circ \Lambda^{\perp} \subseteq \mathfrak{o}
$$

i.e. $\Gamma$ is an $G_{n}^{+}$-invariant integral o-lattice. Furthermore,

$$
2 \Gamma^{\perp}=\sqrt{2} \Lambda \subseteq \sqrt{2} \Lambda^{\perp}=\Gamma,
$$

and

$$
\left(\Gamma^{\perp}: \Gamma\right)=\left(\frac{1}{\sqrt{2}} \Lambda: \sqrt{2} \Lambda^{\perp}\right)=\left(\Lambda: 2 \Lambda^{\perp}\right)=\frac{\left(\Lambda^{\perp}: 2 \Lambda^{\perp}\right)}{\left(\Lambda^{\perp}: \Lambda\right)}=2^{2 N-k} .
$$

This computation shows that, after replacing $\Lambda$ by $\Gamma$ if necessary, one may suppose that $0 \leq k \leq N$. Claim that the last condition implies $k=0,1,2$. For $F=$ $\left(\Lambda \cap 2 \Lambda^{\#}\right) / 2 \Lambda$ is a $G_{n}^{+}$-submodule of $\Lambda / 2 \Lambda$, and $|F|=2^{k}$. By Proposition 2.2 (ii), $k=0,1$, or 2 .

We have arrived at the situation where $\left(\Lambda^{\perp}: \Lambda\right)=2^{k}, k=0,1,2$. By Lemma 2.8, $k \neq 1$. If $k=0$, then $\Lambda=\Lambda^{\perp}=\theta \Lambda^{\#}$, and $\Lambda$ is a $p$-modular Euclidean lattice of rank $2 N$, and we are done. Suppose $k=2$. Then the discriminant group $\Lambda^{\#} / \Lambda$ is isomorphic to $\left(C_{2}\right)^{2} \oplus\left(C_{p}\right)^{N}$, and $\left(\Lambda^{\perp}: \Lambda\right)=\left(C_{2}\right)^{2}$. By Lemma 2.2 of [SchT], there exists a vector $v \in \Lambda^{\perp} \backslash \Lambda$ with $2 v \in \Lambda$ such that $\Delta=\langle\Lambda, v\rangle_{\mathbb{Z}}$ is a $G_{n}^{+}$invariant Euclidean lattice and $\Delta^{\#} / \Delta \simeq\left(C_{p}\right)^{N}$. Remark that $\theta \Delta \subseteq \Delta$. Indeed, 
$\theta v=\pi \cdot 2 v-v \in \Delta$. Furthermore, $\theta \Delta \subseteq p \Delta^{\#}$. (For

$$
(\theta \Delta, \Delta) \subseteq(\theta \Lambda, \Lambda)+(\theta \Lambda, v)+(\Lambda, \theta v)+(\theta v, v) .
$$

Here, $\theta \Lambda \subseteq \theta \Lambda^{\perp}=p \Lambda^{\#}$, so $(\theta \Lambda, \Lambda) \subseteq p \mathbb{Z}$. As $v \in \Lambda^{\perp}$, we have $\Lambda \circ v \subseteq \mathfrak{o}$, and so

$$
(\theta \Lambda, v)=(\Lambda, \theta v) \subseteq \theta \mathfrak{o} \cap \mathbb{Z}=p \mathbb{Z} .
$$

Finally, $(\theta v, v)=\theta v \circ v-\theta v \circ v=0$.) Now we have

$$
(\theta \Delta: p \Delta)=p^{N}=\left(\Delta^{\#}: \Delta\right)=\left(p \Delta^{\#}: p \Delta\right)
$$

therefore in fact $\theta \Delta=p \Delta^{\#}, \Delta=\theta \Delta^{\#}$. The map $f: x \mapsto \theta x$, where $x \in \Delta^{\#}$, maps $\Delta^{\#}$ onto $\Delta$ and preserves $(\cdot, \cdot)$ up to the scalar $p:(f(x), f(y))=p(x, y)$. This means the lattice $\Delta$ is $p$-modular, as desired.

The proof of Theorem 1.2 is finished.

Remark 2.10. Observe that all the lattices $\Delta=\Delta(p, n), \Delta^{-}(p, n)$, are symplectic (for the definition of symplectic lattices cf. [BuS]). For suppose first that $\Delta$ is invariant under $G_{n}^{-}$. Then $\operatorname{det} \Delta=1$. Taking $\tau=\left(\begin{array}{cc}0 & E_{n} \\ \theta E_{n} & 0\end{array}\right)$ (in the chosen symplectic basis of $W), \mathbb{F}_{p}^{\bullet}=\langle\theta\rangle$, one sees that $\tau \in G_{n}^{-}$and $\tau^{2}=\theta E_{2 n}$ acts on $\Delta$ as -1 . According to [BuS], this means that $\Delta$ is symplectic. Suppose now that $\Delta$ is invariant under $G_{n}^{+}$. If $p \equiv 3 \bmod 4$, then we put $\tau=\frac{1}{\sqrt{p}} \sigma$. Considered under the new scalar product $(u, v)^{\prime}=(u, v) / \sqrt{p}$, the dual lattice of $\Delta$ equals $\tau(\Delta)$. Also, $\tau$ preserves $(\cdot, \cdot)^{\prime}$ and $\tau^{2}=-1$. Hence $\Delta$ is symplectic. Finally, let $p \equiv 1 \bmod 4$. Taking $g=\left(\begin{array}{cc}0 & E_{n} \\ \theta E_{n} & 0\end{array}\right)$, one sees that $g \in G_{n}^{+} \backslash S_{n}$. Put $\tau=\frac{1}{\sqrt{p}} g \sigma$ and consider $\Delta$ w.r.t. the new scalar product $(\cdot, \cdot)^{\prime}$ introduced above. Clearly, $\tau$ preserves $(\cdot, \cdot)^{\prime}$ and sends $\Delta$ to its dual (w.r.t. the new scalar product). Besides, $g \in G_{n}^{+} \backslash S_{n}$ implies that $\tau^{2}=-\frac{1}{p} g^{2} \sigma^{2}=-1$. Consequently, $\Delta$ is symplectic.

Remark 2.11. One could formalize Lemmas 2.8, 2.9 and the above arguments in order to get an analogue of Proposition 2.4 in [SchT] for the existence of $p$-modular lattices. Here is one more well known example. Let $G={ }^{2} G_{2}(3)=S L_{2}(8) \cdot C_{3}$ and $\chi$ an irreducible complex character of $G$ of degree 7 with $\mathbb{Q}(\chi)=\mathbb{Q}(\sqrt{-3})$. Then for any prime $r \neq 2,3, \chi \bmod r$ is an irreducible Brauer character, which is not of quadratic type. Furthermore, $\chi$ mod 3 is irreducible and of quadratic type. Finally, $\chi \bmod 2$ is a sum of a character of degree 1 and an irreducible Brauer character of degree 6 which is of symplectic type. From this it follows that $G$ has an irreducible $\mathbb{Q}$-module $V$ with character $\chi+\bar{\chi}$. The above arguments show that $G$ stabilizes a 3-modular lattice $\Lambda$ in $V$ with $\operatorname{Aut}(\Lambda)=C_{2} \times G_{2}(3)$. The lattice $\Lambda$ occurs in [Atlas] and was investigated in detail in [KoT], Chapter 8. It is the unique extremal 3 -modular lattice in dimension 14, after [SchHem].

\section{Explicit CONSTRUCTION. I: $n$ ODD}

We maintain the notation $W$ for the natural $\mathbb{F}_{p} S_{n}$-module $\mathbb{F}_{p}^{2 n}$ endowed with a non-degenerate symplectic form $\langle\cdot, \cdot\rangle$. Throughout this section we suppose that $n$ is odd. We use the ideas of [SchT], $\S 3$ to explicitly construct $G_{n}^{-}$-invariant lattices in $V=V_{n}$, for any odd prime $p$.

Consider an arbitrary $G_{n}^{-}$-invariant (integral) lattice $\Lambda$ in $V$. Fix a symplectic basis $\left(e_{1}, \ldots, e_{n}, f_{1}, \ldots, f_{n}\right)$ of $W$. Recall that $G_{n}$ is generated by $S_{n}$ and the 
element $\vartheta_{n}$ with matrix $\operatorname{diag}\left(E_{n}, \theta E_{n}\right)$ in this basis. We shall view $V$ as a $G_{n^{-}}$ module with kernel $Z^{2} \simeq C_{(p-1) / 2}$. A Lagrangian is a maximal totally isotropic subspace in $W$. Following $[\mathrm{BaV}]$, we consider them oriented, i.e. equipped with an appropriate equivalence class of bases. Two bases $\left(l_{1}, \ldots, l_{n}\right)$ and $\left(l_{1}^{\prime}, \ldots, l_{n}^{\prime}\right)$ of a Lagrangian $L$ are equivalent, i.e. define the same orientation, if the element $g \in G L_{n}(L)$ defined by $g\left(l_{i}\right)=l_{i}^{\prime}, 1 \leq i \leq n$, has $\operatorname{det} g \in \mathbb{F}_{p}^{\bullet 2}$. We denote by $\mathcal{L}(W)$ the set of all oriented Lagrangians contained in $W$.

To each Lagrangian $L$ of $W$ we now associate the following two subgroups:

$$
G(L)=\left\{\varphi \in G_{n} \mid \varphi(L)=L\right\}, S(L)=\left\{\varphi \in G(L) \mid \operatorname{det}\left(\left.\varphi\right|_{L}\right) \in \mathbb{F}_{p}^{\bullet 2}\right\} .
$$

Since the determinant of $\lambda E_{2 n}\left(\lambda \in \mathbb{F}_{p}^{\bullet 2}\right)$ acting on any Lagrangian $L$ is a square in $\mathbb{F}_{p}$, the definition (5) of $S(L)$ factors through the kernel $Z^{2}$ of $\chi$. Let $\xi_{L}$ denote the linear character of $G(L)$ (and $S(L)$ ) which sends $g \in G(L)$ to $\left(\frac{\operatorname{det}\left(\left.\varphi\right|_{W / L}\right)}{p}\right)$. Here and hereafter, $(\dot{\bar{p}})$ stands for the Legendre symbol.

Proposition 3.1. Let $n$ be odd. For any Lagrangian $L$ in $W$, the sets

$$
\begin{gathered}
\Lambda(L)=\{v \in \Lambda \mid \forall \varphi \in S(L), \varphi(v)=v\}, \\
\Lambda^{-}(L)=\left\{v \in \Lambda \mid \forall \varphi \in S(L), \varphi(v)=\xi_{L}(g) v\right\}
\end{gathered}
$$

are 1-dimensional $\mathbb{Z}$-modules.

Proof. 1) Without loss of generality, one can take $L=\left\langle e_{1}, \ldots, e_{n}\right\rangle_{\mathbb{F}_{p}}$ with the basis $\left(e_{1}, \ldots, e_{n}\right)$. Denote

$$
P=S t_{S}(L)=E \cdot H, Q=S(L), R=P \cap Q=E \cdot H^{\bullet},
$$

where $E=\left(C_{p}\right)^{n(n+1) / 2}, H=G L_{n}(p), H^{\bullet}=\left\{g \in G L_{n}(p) \mid \operatorname{det} g \in \mathbb{F}_{p}^{\bullet 2}\right\}$. A model for the Weil representation of $S$ with character $\psi$ is described in [Gro]. From this description it follows that $\left.\psi\right|_{P}=\delta+\zeta$, where $\zeta$ is a $P$-character of degree $\left(p^{n}-1\right) / 2$ and

$$
\delta(\varphi)=\left(\frac{\operatorname{det}\left(\left.\varphi\right|_{L}\right)}{p}\right)
$$

for $\varphi \in P$. In particular, $\left.\psi\right|_{R}=1_{R}+\left.\zeta\right|_{R}$.

If $n=1$, one directly checks that the trivial character of $S(L)$ and the character $\xi_{L}$ each enter into $\left.\chi\right|_{S(L)}$ with multiplicity 1.

2) In this paragraph we suppose $n>1$. We claim that $\left.\zeta\right|_{R} \in \operatorname{Irr}(R)$. Indeed, one can identify $E$ with the space of symmetric matrices of degree $n$ over $\mathbb{F}_{p}$. Furthermore, $P / E=H$ acts on $E$ by the rule:

$$
A \circ X=A \cdot X \cdot{ }^{t} A
$$

for $A \in H$ viewed as an element of $G L_{n}(p)$ and $X \in E$. Obviously $E \nsubseteq \operatorname{Ker} \zeta$. So it is sufficient to show that every $R / E$-orbit on the set $\operatorname{Irr}(E) \backslash\left\{1_{E}\right\}$ has length $\geq\left(p^{n}-1\right) / 2$, or equivalently, every $H^{\bullet}$-orbit on the set $E^{*} \backslash\{0\}$ has length $\geq$

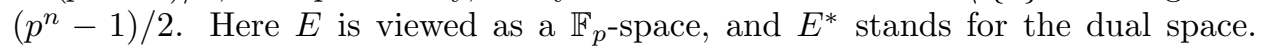
Actually, one can identify the $G L_{n}(p)$-module $E^{*}$ with $E$ itself, but endowed with the action $A \bullet X={ }^{t} A^{-1} \cdot X \cdot A^{-1}$, where $A \in G L_{n}(p), X \in E$. (Indeed, each element $f \in E^{*}$ can be realized as the map $f=f_{M}: X \mapsto \operatorname{Tr}(X \cdot M)$ for a uniquely determined $M \in E$. Now we can write down the action of $A \in G L_{n}(p)$ on $E^{*}$ :

$$
\begin{aligned}
(A \bullet f)(X) & =f_{M}\left(A^{-1} \circ X\right)=\operatorname{Tr}\left(A^{-1} \cdot X \cdot{ }^{t} A^{-1} \cdot M\right) \\
& \left.=\operatorname{Tr}\left(X \cdot{ }^{t} A^{-1} \cdot M \cdot A^{-1}\right)=f_{A \bullet M}(X) .\right)
\end{aligned}
$$


Consider a $G L_{n}(p)$-orbit $\mathcal{O}$ on $E \backslash\{0\}$ and $X \in \mathcal{O}$. Then the stabilizer $H(X)$ of $X$ in $G L_{n}(p)$ is nothing else but the isometry group of the symmetric bilinear form on $\mathbb{F}_{p}^{n}$ with the matrix $X$. It is not difficult to show that the cardinality of $\mathcal{O}$ is $\left(p^{n}-1\right) / 2$ if $\operatorname{rank} X=1$, and strictly greater than $\left(p^{n}-1\right) / 2$ if $\operatorname{rank} X>1$ (and greater than $p^{n}-1$ if $\operatorname{rank} X=n>1$ ). On the other hand, if $\operatorname{rank} X \leq n-1$, then $H(X)$ contains an element $A$ not contained in $H^{\bullet}$, whence $\mathcal{O}$ is also an $H^{\bullet}$-orbit. Therefore, an $H^{\bullet}$-orbit in $E \backslash\{0\}$ can have length less than $\left(p^{n}-1\right) / 2$ only in case $n=1$. (When $n=1$, any $H^{\bullet}$-orbit in $E \backslash\{0\}$ has length $\left(p^{n}-1\right) / 4$.) Now $n>1$ by our assumption, so our claim has been proved.

Decompose $V \otimes_{\mathbb{Q}} \mathbb{C}$ into a sum $U \oplus U_{1} \oplus U_{2}$ of three $R$-submodules, with character $2 \cdot 1_{R}, \zeta$ and $\bar{\zeta}$, respectively. Remark that $R$ contains a regular unipotent element $x$ and $\zeta(x)=\left(-1 \pm p^{n-1} \sqrt{\epsilon p}\right) / 2$. Furthermore, $Q=\left\langle R, \vartheta_{n}\right\rangle$, and $\vartheta_{n}$ normalizes $R$. Therefore $\vartheta_{n}$ fixes $U$, and either leaves both $U_{1}, U_{2}$ invariant or interchanges them. But $\vartheta_{n}$ interchanges the $S$-conjugacy classes of $x$ and $x^{\cdots}$ (some power of $x$ ), and $\zeta\left(x^{\cdots}\right)=\overline{\zeta(x)} \neq \zeta(x)$. This means $\vartheta_{n}$ interchanges $U_{1}$ and $U_{2}$. The construction of $\chi$ (see the proof of Lemma 2.1) ensures that $\chi\left(\vartheta_{n}\right)=0$. As a consequence, $\vartheta_{n}$ acting on $U$ has trace 0 . Observe that $\vartheta_{n}^{2}$ leaves $U$ pointwise fixed. (Indeed, $\vartheta_{n}^{2}$ is the product of $\alpha=\operatorname{diag}\left(\theta^{-1} E_{n}, \theta E_{n}\right)$ and $\beta=\theta E_{2 n}$. Clearly, $\alpha$ belongs to $P$ and acts on $U$ as multiplication by $\delta(\alpha)=\left(\frac{\theta^{-n}}{p}\right)=-1$, because $n$ is odd. Furthermore, $\beta$ acts as multiplication by -1 on the whole of $V$.) We have shown that both of the subspaces

$$
\begin{gathered}
\widetilde{F}=\left\{v \in V \otimes_{\mathbb{Q}} \mathbb{C} \mid \forall \varphi \in Q, \varphi(v)=v\right\}=U \cap \operatorname{Ker}\left(\vartheta_{n}-1\right), \\
\widetilde{F}^{-}=\left\{v \in V \otimes_{\mathbb{Q}} \mathbb{C} \mid \forall \varphi \in Q, \varphi(v)=\xi_{L}(g) v\right\}=U \cap \operatorname{Ker}\left(\vartheta_{n}+1\right)
\end{gathered}
$$

have dimension 1 .

3) For any odd $n$, Lemma 3 of $[\mathrm{CoT}]$ now implies that both of the subspaces

$$
\begin{gathered}
F=\{v \in V \mid \forall \varphi \in Q, \varphi(v)=v\}, \\
F^{-}=\left\{v \in V \mid \forall \varphi \in Q, \varphi(v)=\xi_{L}(g) v\right\}
\end{gathered}
$$

also have dimension 1 (over $\mathbb{Q}$ ). Since $V=\Lambda \otimes_{\mathbb{Z}} \mathbb{Q}$, we arrive at the conclusion that $\Lambda(L)$ and $\Lambda^{-}(L)$ are 1 -dimensional $\mathbb{Z}$-modules.

Keeping Proposition 3.1 in mind, we denote by $v(L)$ (resp. $u(L)$ ) a generating element of the $\mathbb{Z}$-module $\Lambda(L)$ (resp. $\Lambda^{-}(L)$ ) for Lagrangian $L$. Then $v(L)$ (resp. $u(L))$ is determined uniquely up to sign. It is clear that $\Lambda(L)$ and $\Lambda^{-}(L)$ are stabilized by $G(L)$. Namely,

$$
\varphi(v(L))=\left(\frac{\operatorname{det}\left(\left.\varphi\right|_{L}\right)}{p}\right) \cdot v(L), \varphi(u(L))=\left(\frac{\operatorname{det}\left(\left.\varphi\right|_{W / L}\right)}{p}\right) \cdot u(L)
$$

for $\varphi \in G(L)$. Since we consider Lagrangians oriented, we can set $v(-L)=-v(L)$, $u(-L)=-u(L)$ for the opposite Lagrangian $-L$ corresponding to a given oriented Lagrangian $L$. We fix an oriented Lagrangian $L_{0}$ with a basis $\left(e_{1}, \ldots, e_{n}\right)$, and fix a generating vector $v\left(L_{0}\right)$ of $\Lambda\left(L_{0}\right)$ (resp. $u\left(L_{0}\right)$ of $\Lambda^{-}\left(L_{0}\right)$ ). For an arbitrary oriented Lagrangian $M$ with a basis $\left(f_{1}, \ldots, f_{n}\right)$, we find an element $\nu_{M} \in G$ such that $\nu_{M}\left(e_{i}\right)=f_{i}$ for all $i$, and set $v(M)=\nu_{M}\left(v\left(L_{0}\right)\right), u(M)=\nu_{M}\left(u\left(L_{0}\right)\right)$. This definition is independent of the choice of $\nu_{M}$. Moreover, for any $h \in G$ with 
$h\left(L_{0}\right)=M$, we have

$$
h\left(v\left(L_{0}\right)\right)=\left(\frac{\operatorname{det}\left(\left.\left(\nu_{M}^{-1} h\right)\right|_{L_{0}}\right)}{p}\right) \cdot v(M), h\left(u\left(L_{0}\right)\right)=\left(\frac{\operatorname{det}\left(\left.\left(\nu_{M}^{-1} h\right)\right|_{W / L_{0}}\right)}{p}\right) \cdot u(M) .
$$

Lemma 3.2. Let $L$ and $M$ be arbitrary Lagrangians. Then $|(v(L), v(M))|$ (resp. $|(u(L), u(M))|,|(u(L), v(M))|)$ depends only on the dimension of $L \cap M$ (and on the choice of the norm $(v(L), v(L)))$. In other words, there exist non-negative constants $a_{k}, b_{k}, c_{k}, k=0,1, \ldots, n$, such that $|(v(L), v(M))|=a_{k},|(u(L), u(M))|=b_{k}$, $|(u(L), v(M))|=c_{k}$ whenever $\operatorname{dim}(L \cap M)=k$.

Proof. Consider Lagrangians $L^{\prime}, M^{\prime}$ with $\operatorname{dim}(L \cap M)=\operatorname{dim}\left(L^{\prime} \cap M^{\prime}\right)$. It is clear that there exists an element $\varphi \in S$ mapping $L$ into $L^{\prime}$ and $M$ into $M^{\prime}$. One readily verifies that $\varphi S(L) \varphi^{-1}=S\left(L^{\prime}\right)$. Taking $g \in S(L)$ and applying (6) we have

$$
g \varphi^{-1}\left(v\left(L^{\prime}\right)\right)=\varphi^{-1} \cdot \varphi g \varphi^{-1}\left(v\left(L^{\prime}\right)\right)=\varphi^{-1}\left(v\left(L^{\prime}\right)\right)
$$

for each $g \in S(L)$. By Proposition 3.1 this implies that $\varphi^{-1}\left(v\left(L^{\prime}\right)\right)= \pm v(L)$, i.e. $\varphi(v(L))= \pm v\left(L^{\prime}\right)$. Similarly, $\varphi(v(M))= \pm v\left(M^{\prime}\right)$. In particular, $\left(v\left(L^{\prime}\right), v\left(M^{\prime}\right)\right)=$ $\pm(v(L), v(M))$. Next we have $\xi_{L}(g)=\xi_{L^{\prime}}\left(\varphi g \varphi^{-1}\right)$, and

$$
g \varphi^{-1}\left(u\left(L^{\prime}\right)\right)=\varphi^{-1} \cdot \varphi g \varphi^{-1}\left(u\left(L^{\prime}\right)\right)=\varphi^{-1}\left(\xi_{L^{\prime}}\left(\varphi g \varphi^{-1}\right) u\left(L^{\prime}\right)\right)=\xi_{L}(g) \varphi^{-1}\left(u\left(L^{\prime}\right)\right) .
$$

By Proposition 3.1 this implies that $\varphi^{-1}\left(u\left(L^{\prime}\right)\right)= \pm u(L)$, i.e. $\varphi(u(L))= \pm u\left(L^{\prime}\right)$. Similarly, $\varphi(u(M))= \pm u\left(M^{\prime}\right)$. Hence,

$$
\left(u\left(L^{\prime}\right), u\left(M^{\prime}\right)\right)= \pm(u(L), u(M)),\left(u\left(L^{\prime}\right), v\left(M^{\prime}\right)\right)= \pm(u(L), v(M)) .
$$

Lemma 3.3. If $k$ is even, then $a_{k}=0$. If $k$ is odd, then $c_{k}=0$.

Proof. Again consider the symplectic basis $\left(e_{1}, \ldots, e_{n}, f_{1}, \ldots, f_{n}\right)$. If the intersection of given Lagrangians $L, L^{\prime}$ has dimension $k, k$ a non-negative integer, then without loss of generality one can suppose that

$$
L=\left\langle e_{1}, \ldots, e_{n}\right\rangle_{\mathbb{F}_{p}}, L^{\prime}=\left\langle e_{1}, \ldots, e_{k}, f_{k+1}, \ldots, f_{n}\right\rangle_{\mathbb{F}_{p}}
$$

Clearly that $\vartheta_{n}$ is contained in both of $G(L), G\left(L^{\prime}\right)$. Furthermore, $\operatorname{det}\left(\left.\vartheta_{n}\right|_{L}\right)=1$, $\operatorname{det}\left(\left.\vartheta_{n}\right|_{W / L}\right)=\theta^{n}$, and $\operatorname{det}\left(\left.\vartheta_{n}\right|_{L^{\prime}}\right)=\theta^{n-k}$.

First suppose that $k$ is even. Due to (6) one then has $\vartheta_{n}(v(L))=v(L)$, $\vartheta_{n}\left(v\left(L^{\prime}\right)\right)=-v\left(L^{\prime}\right)$. Therefore,

$$
\left(v(L), v\left(L^{\prime}\right)\right)=\left(\vartheta_{n}(v(L)), \vartheta_{n}\left(v\left(L^{\prime}\right)\right)\right)=-\left(v(L), v\left(L^{\prime}\right)\right),
$$

i.e. $\left(v(L), v\left(L^{\prime}\right)\right)=0$.

Next suppose that $k$ is odd. Then due to (6) one has $\vartheta_{n}(u(L))=-u(L)$, $\vartheta_{n}\left(v\left(L^{\prime}\right)\right)=v\left(L^{\prime}\right)$. Now we get

$$
\left(u(L), v\left(L^{\prime}\right)\right)=\left(\vartheta_{n}(u(L)), \vartheta_{n}\left(v\left(L^{\prime}\right)\right)\right)=-\left(u(L), v\left(L^{\prime}\right)\right),
$$

i.e. $\left(u(L), v\left(L^{\prime}\right)\right)=0$.

Our next goal is to determine $a_{k}$ for $k$ odd, and $c_{k}$ for $k$ even. Recall that a symplectic spread of $W$ is a collection $\pi=\left\{W_{i} \mid 1 \leq i \leq p^{n}+1\right\}$ consisting of $p^{n}+1$ maximal totally isotropic subspaces such that $\bigcup_{i=1}^{p^{n}+1} W_{i}=W$. The socalled standard, or desarguesian, symplectic spread of $W$ can be constructed in the following way. Identify $W$ with $\mathbb{F}_{q}^{2}, q=p^{n}$, and endow $W$ with the symplectic 
form $\langle u, v\rangle=\operatorname{tr}(\alpha \delta-\beta \gamma)$, where $u=(\alpha, \beta), v=(\gamma, \delta), \alpha, \beta, \gamma, \delta \in \mathbb{F}_{q}$, and $\operatorname{tr}$ stands for the trace form $\operatorname{tr}: \mathbb{F}_{q} \rightarrow \mathbb{F}_{p}$. Then

$$
\pi_{D}=\left\{W^{\lambda} \mid \lambda \in \mathbb{F}_{q} \cup\{\infty\}\right\},
$$

where $W^{\infty}=\left\{(0, \alpha) \mid \alpha \in \mathbb{F}_{q}\right\}, W^{\lambda}=\left\{(\alpha, \lambda \alpha) \mid \alpha \in \mathbb{F}_{q}\right\}$ for $\lambda \in \mathbb{F}_{q}$ is the desired spread. One may suppose that

$$
W^{0}=\left\langle e_{1}, \ldots, e_{n}\right\rangle_{\mathbb{F}_{p}}, W^{\infty}=\left\langle f_{1}, \ldots, f_{n}\right\rangle_{\mathbb{F}_{p}} .
$$

For a given symplectic spread $\pi=\left\{W_{i}\right\}$, its automorphism group Aut $(\pi)$ is defined as the group $\left\{\varphi \in C S p_{2 n}(p) \mid \forall i \exists j\right.$ s.t. $\left.\varphi\left(W_{i}\right)=W_{j}\right\}$. For example (see [KoT], Lemma 1.2.6),

$$
\operatorname{Aut}\left(\pi_{D}\right)=S L_{2}(q) \cdot C_{n} \cdot C_{p-1},
$$

the extension of $S L_{2}(q)$ first by the Galois group of the extension $\mathbb{F}_{q} / \mathbb{F}_{p}$ and then by the element $\vartheta_{n}$. Set

$$
\Lambda(\pi)=\langle v(L) \mid L \in \pi\rangle_{\mathbb{Z}}
$$

Then, by Lemma 3.3, $\Lambda(\pi)$ is a sublattice of $\Lambda$ of determinant $\left(a_{n}\right)^{p^{n}+1}$, where $a_{n}=(v(L), v(L))$ as in Lemma 3.2. In particular,

$$
\operatorname{det} V=\mathbb{Q}^{\bullet 2}
$$

the fact we used in the proof of Theorem 1.1. Also, it shows that $V$ contains no $p$-modular lattices (if $p^{n} \equiv 1 \bmod 4$ ).

Now we consider the standard symplectic spread $\pi_{D}$, and project $v(M)$ and $u(M), M$ a fixed Lagrangian, to the orthogonal basis $\left(v\left(W^{\lambda}\right)\right)$ :

$$
v(M)=\sum_{\lambda \in \mathbb{F}_{q} \cup\{\infty\}} z_{\lambda} v\left(W^{\lambda}\right), u(M)=\sum_{\lambda \in \mathbb{F}_{q} \cup\{\infty\}} y_{\lambda} v\left(W^{\lambda}\right) .
$$

It is obvious that $z_{\lambda}=a_{n}^{-1}\left(v(M), v\left(W^{\lambda}\right)\right), y_{\lambda}=a_{n}^{-1}\left(u(M), v\left(W^{\lambda}\right)\right)$, and so

$$
\sum_{\lambda \in \mathbb{F}_{q} \cup\{\infty\}}\left(v(M), v\left(W^{\lambda}\right)\right)^{2}=a_{n}^{2}, \sum_{\lambda \in \mathbb{F}_{q} \cup\{\infty\}}\left(u(M), v\left(W^{\lambda}\right)\right)^{2}=a_{n} b_{n} .
$$

Proposition 3.4. In the notation of Lemma 3.2 one has

(i) $a_{k}=p^{-(n-k) / 2} \cdot a_{n}$ for odd $k, 1 \leq k \leq n$;

(ii) $\left(c_{k}\right)^{2}=p^{k-n} \cdot a_{n} b_{n}$ for even $k, 0 \leq k<n$.

Proof. We shall proceed by induction on $n=1,3, \ldots$.

1) Applying (8) to $M=W^{\infty}$ we get $a_{n} b_{n}=p^{n}\left(c_{0}\right)^{2}$. Next we take $M=$ $\left\langle e_{1}, f_{2}, \ldots, f_{n}\right\rangle_{\mathbb{F}_{p}}$ and write $e_{1}=(e, 0)$ for $e \in \mathbb{F}_{q}^{\bullet}$. Then $M \cap W^{\infty}=\left\langle f_{2}, \ldots, f_{n}\right\rangle$ has dimension $n-1$. Furthermore, for an arbitrary $\lambda \in \mathbb{F}_{q}$ one has

$$
\begin{aligned}
M \cap W^{\lambda} & =\left\{(x e, \lambda x e) \mid x \in \mathbb{F}_{p},\left\langle(0, \lambda e), e_{1}\right\rangle=0\right\} \\
& =\left\{(x e, \lambda x e) \mid x \in \mathbb{F}_{p}, \operatorname{tr}\left(\lambda e^{2}\right)=0\right\} .
\end{aligned}
$$

Therefore, $\operatorname{dim}\left(M \cap W^{\lambda}\right)$ is equal to 1 for just $p^{n-1}$ values of $\lambda \in \mathbb{F}_{q}$, and 0 for the other $\lambda$ 's. Applying (8), one has $p^{n-1} a_{1}^{2}=a_{n}^{2}$, i.e. $a_{1}=p^{-(n-1) / 2} a_{n}$. Thus we have proved Proposition 3.4 for $a_{1}$ and $c_{0}$ with $n \geq 1$. In particular, the induction base $n=1$ has been established.

2) For the induction step we suppose $n \geq 3$. We already proved the desired relations for $a_{1}$ and $c_{0}$. Put

$$
W^{\prime}=\left\langle e_{1}, \ldots, e_{n-2}, f_{1}, \ldots, f_{n-2}\right\rangle_{\mathbb{F}_{p}}, W^{\prime \prime}=\left\langle e_{n-1}, e_{n}, f_{n-1}, f_{n}\right\rangle_{\mathbb{F}_{p}},
$$


$U=\left\langle e_{n-1}, e_{n}\right\rangle_{\mathbb{F}_{p}}$, and introduce the following subgroups in $S: B=S t_{S}\left(W^{\prime}\right)$, $S^{\prime}=\left\{\varphi \in B|\varphi|_{W^{\prime \prime}}=1_{W^{\prime \prime}}\right\}, S^{\prime \prime}=\left\{\varphi \in B|\varphi|_{W^{\prime}}=1_{W^{\prime}}\right\}, C=S^{\prime \prime} \cap S t_{S}(U)$, $K=S^{\prime} \times C$. Then $S^{\prime} \simeq S p\left(W^{\prime}\right)=S p_{2 n-4}(p), S^{\prime \prime} \simeq S p\left(W^{\prime \prime}\right)=S p_{4}(p), C \simeq$ $\left(C_{p}\right)^{3} \cdot G L_{2}(p), B=S^{\prime} \times S^{\prime \prime}$. We also set $G^{\prime}=\left\langle S^{\prime}, \vartheta_{n}\right\rangle, H=\left\langle K, \vartheta_{n}\right\rangle=G^{\prime} \cdot C$. It is well known that $\left.\psi\right|_{B}=\psi^{\prime} \otimes \psi^{\prime \prime}+\tau^{\prime} \otimes \tau^{\prime \prime}$, where $\psi^{\prime}$ (resp. $\tau^{\prime}$ ) is an irreducible Weil character of $S^{\prime}$ of degree $\left(p^{n-2}+1\right) / 2$ (resp. $\left.\left(p^{n-2}-1\right) / 2\right)$. Furthermore, $\psi^{\prime \prime}\left(\right.$ resp. $\left.\tau^{\prime \prime}\right)$ is an irreducible Weil character of $S^{\prime \prime}$ of degree $\left(p^{2}+1\right) / 2$ (resp. $\left.\left(p^{2}-1\right) / 2\right)$. Arguing as in the proof of Proposition 3.1, we are convinced that $\alpha:=\left.\tau^{\prime \prime}\right|_{C} \in \operatorname{Irr}(C)$, and $\left.\psi^{\prime \prime}\right|_{C}=\delta+\beta$, where $\beta \in \operatorname{Irr}(C)$ and $\delta(\varphi)=\left(\frac{\operatorname{det}\left(\left.\varphi\right|_{U}\right)}{p}\right)$ for $\varphi \in C$. (In particular, $\delta(1)=1$.) Thus

$$
\left.\psi\right|_{K}=\psi^{\prime} \otimes \delta+\psi^{\prime} \otimes \beta+\tau^{\prime} \otimes \alpha
$$

is a sum of three (pairwise distinct) irreducible constituents. From this it follows that

$$
\left.\chi\right|_{K}=\left(\psi^{\prime}+\overline{\psi^{\prime}}\right) \otimes \delta+\left(\psi^{\prime} \otimes \beta+\overline{\psi^{\prime}} \otimes \bar{\beta}\right)+\left(\tau^{\prime} \otimes \alpha+\overline{\tau^{\prime}} \otimes \bar{\alpha}\right) .
$$

Observe that $\vartheta_{n}$ acts on $S^{\prime}$ as an outer automorphism, and $\vartheta_{n}$ interchanges the characters $\psi^{\prime}$ and $\overline{\psi^{\prime}}$. Furthermore, $C \triangleleft H$. Consequently, $\left.\chi\right|_{H}$ has a unique irreducible constituent in which $C$ acts by scalars. This constituent affords $K$ character $\left(\psi^{\prime}+\overline{\psi^{\prime}}\right) \otimes \delta$. Also,

$$
\left(\left.\chi\right|_{C}, \delta\right)_{C}=p^{n-2}+1
$$

3) Next we consider the following $\mathbb{Z}$-submodule:

$$
\left.\Lambda^{\prime}=\langle v(L)| L=L^{\prime} \oplus U, L^{\prime} \text { Lagrangian in } W^{\prime}\right\rangle_{\mathbb{Z}}
$$

in $\Lambda$. (The symplectic form on $W^{\prime}$ is inherited from the one on $W$.) Clearly, $H$ leaves $\Lambda^{\prime}$ fixed. Moreover, let $L=L^{\prime} \oplus U, L^{\prime}$ a Lagrangian in $W^{\prime}$ and $\varphi \in C$. Then $\varphi(L)=L$. Hence, due to (6) the subgroup $C$ acts on $\Lambda^{\prime}$ as scalars (and the corresponding character is $\operatorname{dim}_{\mathbb{Z}} \Lambda^{\prime} \cdot \delta$ ). By the result of 2 ), $\operatorname{dim}_{\mathbb{Z}} \Lambda^{\prime}=p^{n-2}+1$. Recall that we chose $G^{\prime}$ to be generated by $S^{\prime}=S p\left(W^{\prime}\right)$ and $\vartheta_{n}$. Considering the natural action of $G^{\prime}$ on $W^{\prime}$, we conclude that $G^{\prime} \simeq C S p\left(W^{\prime}\right)$. We want to find the kernel of $G^{\prime}$ acting on $\Lambda^{\prime}$. To this end, consider a generating element $z=\theta E_{2 n-4}$ of the center $C_{p-1}$ of $C S p\left(W^{\prime}\right)$. Then $z$ acting on $W$ has the following matrix: $\operatorname{diag}\left(\theta E_{2 n-4}, E_{2}, \theta^{2} E_{2}\right)$ in the basis $\left(e_{1}, \ldots, e_{n-2}, f_{1}, \ldots, f_{n-2}, e_{n-1}, e_{n}, f_{n-1}, f_{n}\right)$. If $L=L^{\prime} \oplus U$ ( $L^{\prime}$ any Lagrangian in $\left.W^{\prime}\right)$, then due to $(6) z(v(L))=-v(L)$, as $n$ is odd. Thus $z$ acts on $\Lambda^{\prime}$ as multiplication by -1 . We have shown that the lattice $\Lambda^{\prime}$ is in fact acted on by $C S p_{2 n-4}(p) / C_{(p-1) / 2}=G_{n-2}^{-}$, and this action affords $S^{\prime}$-character $\psi^{\prime}+\overline{\psi^{\prime}}$. If we denote $G^{\prime}\left(L^{\prime}\right)=S t_{G^{\prime}}\left(L^{\prime}\right)$, and define $S^{\prime}\left(L^{\prime}\right)$ similarly to (5), then of course $G^{\prime}\left(L^{\prime}\right)=G(L) \cap G^{\prime}, S^{\prime}\left(L^{\prime}\right)=S(L) \cap G^{\prime}$ for $L=L^{\prime} \oplus U$. In other words, $W^{\prime}, \Lambda^{\prime}, L^{\prime}, v^{\prime}\left(L^{\prime}\right)$ and $u^{\prime}\left(L^{\prime}\right)$ (generating vectors of $\Lambda^{\prime}\left(L^{\prime}\right)$ and $\Lambda^{\prime-}\left(L^{\prime}\right)$, cf. Proposition 3.1) play the same roles for $G^{\prime}$ as $W, \Lambda, L, v(L)$ and $u(L)$ do for $G$.

Observe that there are nonzero rational scalars $s$ and $t$ such that $v^{\prime}\left(L^{\prime}\right)=$ $\pm s v(L), u^{\prime}\left(L^{\prime}\right)= \pm t u(L)$. Indeed, $v(L) \in \Lambda^{\prime}$ by the definition of $\Lambda^{\prime}$, and $v(L)$ is obviously fixed by $S^{\prime}\left(L^{\prime}\right)$; hence $v(L) \in \Lambda^{\prime}\left(L^{\prime}\right)$, and $v^{\prime}\left(L^{\prime}\right)= \pm s v(L)$ for some $s \in \mathbb{Q}^{\bullet}$. Next, $\langle u(L)\rangle_{\mathbb{Z}}$ is a $C$-module with character $\delta$ (cf. (6)). On the other hand, $\Lambda^{\prime}$ affords $C$-character $\left(p^{n-2}+1\right) \delta$. Hence by $(9)$ we have $u(L) \in \Lambda^{\prime} \otimes_{\mathbb{Z}} \mathbb{C}$. From this it follows that $u(L) \in \Lambda^{\prime-}\left(L^{\prime}\right) \otimes_{\mathbb{Z}} \mathbb{C}$, i.e. $u^{\prime}\left(L^{\prime}\right)= \pm t u(L)$ for a certain $t \in \mathbb{C}$. Observe that

$$
s t= \pm\left(u^{\prime}\left(L^{\prime}\right), v^{\prime}\left(M^{\prime}\right)\right) /(u(L), v(M))
$$


is a rational number, where $L^{\prime}, M^{\prime}$ are Lagrangians inside $W^{\prime}$ with $\operatorname{dim}\left(L^{\prime} \cap M^{\prime}\right)=$ $n-3$. Hence $t$ is rational. We may suppose that $s, t>0$.

Now we can apply the induction hypothesis to $G^{\prime}$ and $\Lambda^{\prime}$. In doing so we consider two arbitrary Lagrangians $L^{\prime}, M^{\prime}$ of $W^{\prime}$ with $\operatorname{dim}\left(L^{\prime} \cap M^{\prime}\right)=k$. Then for $L=L^{\prime} \oplus U, M=M^{\prime} \oplus U$ one has $\operatorname{dim}(L \cap M)=k+2$, which implies that $a_{k}^{\prime}=\left|\left(v^{\prime}\left(L^{\prime}\right), v^{\prime}\left(M^{\prime}\right)\right)\right|=s^{2}|(v(L), v(M))|=s^{2} a_{k+2}, b_{k}^{\prime}=\left|\left(u^{\prime}\left(L^{\prime}\right), u^{\prime}\left(M^{\prime}\right)\right)\right|=$ $t^{2}|(u(L), u(M))|=t^{2} b_{k+2}, c_{k}^{\prime}=\left|\left(u^{\prime}\left(L^{\prime}\right), v^{\prime}\left(M^{\prime}\right)\right)\right|=s t|(u(L), u(M))|=s t c_{k+2}$. Вy the induction hypothesis, for $k$ odd we have

$$
s^{2} a_{k+2}=a_{k}^{\prime}=p^{(n-2-k) / 2} a_{n-2}^{\prime}=s^{2} p^{(n-(k+2)) / 2} a_{n},
$$

i.e. $a_{k+2}=p^{(n-(k+2)) / 2} a_{n}$. Thus we have proved the desired relation for $a_{l}$ with $l=3,5, \ldots, n$. Similarly, if $k$ is even, then

$$
s^{2} t^{2}\left(c_{k+2}\right)^{2}=\left(c_{k}^{\prime}\right)^{2}=p^{k-n+2} a_{n-2}^{\prime} b_{n-2}^{\prime}=s^{2} t^{2} p^{k-n+2} a_{n} b_{n}
$$

i.e. $\left(c_{k+2}\right)^{2}=p^{k+2-n} a_{n} b_{n}$. Thus we have proved the desired relation for $c_{l}$ with $l=2,4, \ldots, n-1$. The induction step is over.

Corollary 3.5. Rescale the $v(L)$ 's such that $(v(L), v(L))=p^{(n-1) / 2}$. Then

$$
(v(L), v(M))=\left\{\begin{array}{cl} 
\pm p^{(k-1) / 2}, & \operatorname{dim}(L \cap M)=k \equiv 1 \bmod 2, \\
0, & \operatorname{dim}(L \cap M) \equiv 0 \bmod 2 .
\end{array}\right.
$$

The signs \pm involved in this corollary will be determined in $\S 5$, cf. Corollary 5.4.

Now we consider the endomorphism $\sigma$ of $V$ (constructed in Proposition 2.4 for $p \equiv 1 \bmod 4$ and in [SchT], $\S 5$ for $p \equiv 3 \bmod 4$. Recall that $\Lambda$ is a $G$-invariant lattice in $V$. Let $\Gamma$ be the sublattice of $\Lambda$ generated by $v(L)$ with $L$ running over all Lagrangians in $W$. Clearly, one can rescale the scalar product on $V$ such that $\nabla=\Gamma+\sigma(\Gamma)$ is an integral $G$-invariant $\sigma$-stable lattice lying in $V$. Also, $\Gamma(L)=\Lambda(L)$ for any Lagrangian $L$. We can now apply Propositions $3.1,3.4$ and Lemmas $3.2,3.3$ to the lattice $\nabla$. Let $\widetilde{v}(L), \widetilde{u}(L)$ be generating vectors of $\nabla(L)$, $\nabla^{-}(L)$.

Lemma 3.6. For the lattice $\nabla=\Gamma+\sigma(\Gamma)$ we have $\widetilde{v}(L)= \pm v(L)$ and $\widetilde{u}(L)=$ $\pm \sigma(v(L))$. In particular, the parameters $a_{k}, b_{k}, c_{k}$ of $\nabla$ satisfy the following relations:

(i) $b_{k}=p a_{k}$ for any $k$;

(ii) $c_{k}=p^{(k+1-n) / 2} \cdot a_{n}$ for any even $k$.

Proof. Since $\Gamma \subseteq \nabla, v(L)=m \widetilde{v}(L)$ for some integer $m$. As $\Gamma$ is generated by the $v(L)$ 's, $\Gamma \subseteq m \nabla$, and so $\nabla=\Gamma+\sigma(\Gamma) \subseteq m(\nabla+\sigma(\nabla))=m \nabla$, yielding $m= \pm 1$, i.e. $\widetilde{v}(L)= \pm v(L)$.

Recall that $g \sigma=\left(\frac{\operatorname{det}\left(\left.g\right|_{W}\right)}{p}\right) \sigma g$ for any $g \in G$. If $g \in G(L)$, then $\operatorname{det}\left(\left.g\right|_{W}\right)=$ $\operatorname{det}\left(\left.g\right|_{L}\right) \cdot \operatorname{det}\left(\left.g\right|_{W / L}\right)$, and so $\left(\frac{\operatorname{det}\left(\left.g\right|_{W}\right)}{p}\right)=\left(\frac{\operatorname{det}\left(\left.g\right|_{L}\right)}{p}\right) \cdot \xi_{L}(g)$. Therefore, by (6) one has

$$
\begin{gathered}
g \sigma(v(L))=\left(\frac{\operatorname{det}\left(\left.g\right|_{W}\right)}{p}\right) \sigma g(v(L)) \\
=\left(\frac{\operatorname{det}\left(\left.g\right|_{W}\right)}{p}\right) \cdot\left(\frac{\operatorname{det}\left(\left.g\right|_{L}\right)}{p}\right) \sigma(v(L))=\xi_{L}(g) \sigma(v(L)) .
\end{gathered}
$$

This means: $\sigma(v(L)) \in \nabla^{-}(L)$; hence $\sigma(v(L))=k \cdot \widetilde{u}(L)$ for some $k \in \mathbb{Z}$. Similarly,

$$
\begin{gathered}
g \sigma(\widetilde{u}(L))=\left(\frac{\operatorname{det}\left(\left.g\right|_{W}\right)}{p}\right) \sigma g(\widetilde{u}(L)) \\
=\left(\frac{\operatorname{det}\left(\left.g\right|_{W}\right)}{p}\right) \cdot \xi_{L}(g) \sigma(\widetilde{u}(L))=\left(\frac{\operatorname{det}\left(\left.g\right|_{L}\right)}{p}\right) \sigma(\widetilde{u}(L)),
\end{gathered}
$$


which implies that $\sigma(\widetilde{u}(L)) \in \nabla(L)$. From this it follows that $\sigma(\widetilde{u}(L))=l \cdot v(L)$ for some $l \in \mathbb{Z}$. In this case we have

$$
\epsilon p \cdot v(L)=\sigma^{2}(v(L))=\sigma(k \cdot \widetilde{u}(L))=k \sigma(\widetilde{u}(L))=k l \cdot v(L),
$$

i.e. $k l= \pm p$. Assume $k \neq \pm 1$. Then $k= \pm p, l= \pm 1$, and $v(L)= \pm \sigma(\widetilde{u}(L))$ belongs to $\sigma(\nabla)$. Since $\nabla$ is generated by the vectors $v(L)$ and the sublattice $\sigma(\Gamma)$ which is contained in $\sigma(\nabla)$, we conclude that $\nabla \subseteq \sigma(\nabla)$. Applying $\sigma$ once more again, we get $\nabla \subseteq \sigma^{2}(\nabla)=p \nabla$, a contradiction. Hence $k= \pm 1$, i.e. $\widetilde{u}(L)= \pm \sigma(v(L))$.

Next we take $L, M$ such that $\operatorname{dim}(L \cap M)=k$. Then

$$
b_{k}=|(\widetilde{u}(L), \widetilde{u}(M))|=|(\sigma(v(L)), \sigma(v(M)))|=p|(v(L), v(M))|=p a_{k} .
$$

Furthermore, by Proposition 3.4 for even $k$ one has

$$
\left(c_{k}\right)^{2}=p^{k-n} a_{n} b_{n}=p^{k+1-n}\left(a_{n}\right)^{2},
$$

i.e. $c_{k}=p^{(k+1-n) / 2} \cdot a_{n}$.

Remark 3.7. The assumption $\Gamma=\langle v(L)| L$ any Lagrangian $\rangle$ is essential for the conclusions of Lemma 3.6. For example, the parameters $a_{k}, b_{k}$ of the lattice $\sigma(\nabla)$ satisfy $a_{k}=p b_{k}$.

A key ingredient in our further arguments is the following observation:

Proposition 3.8. Let $\left(e_{1}, \ldots, e_{n}, f_{1}, \ldots, f_{n}\right)$ be any arbitrary symplectic basis of $W$, and let $M, L^{\lambda}, \lambda \in \mathbb{F}_{p}$, be Lagrangians with bases $\left(f_{1}, \ldots, f_{n}\right),\left(e_{1}+\lambda f_{1}, f_{2}, \ldots\right.$, $\left.f_{n}\right)$, respectively. Then in the notation of Lemma 3.6 one has

$$
\widetilde{u}(M)=\sum_{\lambda \in \mathbb{F}_{p}} d_{\lambda} v\left(L^{\lambda}\right), p v(M)=\sum_{\lambda \in \mathbb{F}_{p}} d_{\lambda}^{\prime} \widetilde{u}\left(L^{\lambda}\right)
$$

with $d_{\lambda}, d_{\lambda}^{\prime}= \pm 1$.

Proof. Observe that $\operatorname{dim}\left(M \cap L^{\lambda}\right)=n-1$. Hence in accordance with Lemma 3.6 we have $\left(\widetilde{u}(M), v\left(L^{\lambda}\right)\right)=d_{\lambda} a_{n}$ with $d_{\lambda}= \pm 1$. Besides, $(\widetilde{u}(M), \widetilde{u}(M))=p a_{n}$ and $\left(v\left(L^{\lambda}\right), v\left(L^{\lambda^{\prime}}\right)\right)=a_{n} \delta_{\lambda, \lambda^{\prime}}$. Hence, for $v=\widetilde{u}(M)-\sum_{\lambda \in \mathbb{F}_{p}} d_{\lambda} v\left(L^{\lambda}\right)$ we have $(v, v)=2 p a_{n}-2 p a_{n}=0$, yielding $\widetilde{u}(M)=\sum_{\lambda \in \mathbb{F}_{p}} d_{\lambda} v\left(L^{\lambda}\right)$. Applying $\sigma$ to this identity, we obtain $p v(M)=\sum_{\lambda \in \mathbb{F}_{p}} d_{\lambda}^{\prime} \widetilde{u}\left(L^{\lambda}\right)$.

Now we are in a position to explicitly exhibit a $G$-invariant odd unimodular lattice in $V$.

Theorem 3.9. Let $p$ be any odd prime and $n$ any odd integer. For every Lagrangian $L$ in $W$, choose a vector $v(L)$ in $V \otimes_{\mathbb{Q}} \mathbb{R}$ fixed by $S(L)$ and such that $(v(L), v(L))=p^{(n-1) / 2}$. Then the lattice $\Delta=\Delta(p, n)$ generated by all $v(L)$ 's

$$
\Delta=\langle v(L) \mid L \in \mathcal{L}(W)\rangle_{\mathbb{Z}}
$$

is a $\sigma$-stable $G_{n}^{-}$-invariant odd unimodular lattice.

Proof. We start with some $G$-invariant integral lattice $\Lambda$ and choose $v^{\prime}(L)$ to be a generating vector of the $\mathbb{Z}$-module $\Lambda(L), L$ any Lagrangian. Then according to Lemma 3.3 and Proposition 3.4, $\left(v^{\prime}(L), v^{\prime}(M)\right)=0$ if $k=\operatorname{dim}(L \cap M)$ is even, and $\left(v^{\prime}(L), v^{\prime}(M)\right)= \pm p^{(k-1) / 2} a_{1}$ if $k$ is odd. Here $a_{1}$ is some natural integer. Now we set $v(L)=a_{1}^{-1 / 2} v^{\prime}(L)$ for all Lagrangians $L$. Clearly, $v(L) \in V \otimes_{\mathbb{Q}} \mathbb{R}$, $(v(L), v(L))=p^{(n-1) / 2}$ and $v(L)$ is $S(L)$-stable. (We could assume $v(L) \in V$ by means of rescaling the scalar product on $V$ by the scalar $a_{1}^{-1}$.) Furthermore, 
$(v(L), v(M)) \in \mathbb{Z}$ for any $L, M$. We see that $\Delta$ as defined in the theorem is a $G$ invariant integral lattice. Moreover, if $\pi_{D}$ denotes the standard symplectic spread, then $\Delta$ contains the sublattice

$$
\Delta\left(\pi_{D}\right)=\left\langle v(L) \mid L \in \pi_{D}\right\rangle_{\mathbb{Z}}
$$

of determinant $p^{(n-1)\left(p^{n}+1\right) / 2}$. In particular, $\operatorname{det} \Delta$ is a power of $p$ : $\operatorname{det} \Delta=p^{m}$ for some non-negative integer $m$.

If $m=0$, we are done. Suppose that $m \geq 1$. Then consider the form $(\bar{x}, \bar{y})_{p}=$ $(x, y) \bmod p$ on $\Delta / p \Delta$, where $\bar{x}=x+p \Delta, \bar{y}=y+p \Delta$. As $p \operatorname{divides} \operatorname{det} \Delta,(\cdot, \cdot)_{p}$ is degenerate on $\Delta / p \Delta$. This means that $p \Delta$ is a proper sublattice of $\Delta \cap p \Delta^{\#}$. If $\Delta \cap p \Delta^{\#}=\Delta$, then $\Delta \subseteq p \Delta^{\#}$; in particular, $(v(L), v(M)) \in p \mathbb{Z}$ for all $L, M$, contrary to the equality $(v(L), v(M))= \pm 1$ for $\operatorname{dim}(L \cap M)=1$. Therefore, $\Delta \supset \Delta \cap p \Delta^{\#} \supset p \Delta$. One may then suppose that $\Delta /\left(\Delta \cap p \Delta^{\#}\right)$ affords the $G$-character $\eta_{1}$ mentioned in Proposition 2.2 (iii). Since $\Delta /\left(\Delta \cap p \Delta^{\#}\right)$ supports the $G$-invariant non-degenerate symmetric bilinear form $(\cdot, \cdot)_{p}, \eta_{1}$ is of quadratic type, contrary to Proposition 2.2 (iii).

2) By Lemma 3.6 and Proposition 3.8, $\sigma(v(L))$ belongs to $\Delta$ for any $L$. Hence $\Delta$ is $\sigma$-stable.

Corollary 3.10. For the lattice $\Delta=\Delta(p, n)$ and generating vectors $v(L), u(L)$ of $\Delta(L), \Delta^{-}(L)$, we have $u(L)= \pm \sigma(v(L))$. In particular, the parameters $a_{k}, b_{k}, c_{k}$ of $\Delta$ satisfy the following relations:

(i) $b_{k}=p a_{k}$ for any $k$;

(ii) $c_{k}=p^{(k+1-n) / 2} \cdot a_{n}$ for any even $k$.

\section{Explicit CONSTRUCTION. II: $n$ IS EVEN}

Let $p$ be an odd prime and $n$ any even integer. In this section we exploit the results of $\S 3$ to describe an explicit construction of $G_{n}^{+}$-invariant $p$-modular lattices in $V=V_{n}$. Setting $S^{\prime}=S_{n+1}=S p_{2 n+2}(p)$, we consider a natural $\mathbb{F}_{p} S^{\prime}$-module $W^{\prime}=W_{n+1}=\mathbb{F}_{p}^{2 n+2}$ endowed with a non-degenerate symplectic form $\langle\cdot, \cdot\rangle$. Fix some symplectic basis $\left(e_{1}, \ldots, e_{n+1}, f_{1}, \ldots, f_{n+1}\right)$ of $W^{\prime}$. Consider the endomorphism $\vartheta_{n+1}$ of $W^{\prime}$ with the matrix $\operatorname{diag}\left(E_{n+1}, \theta E_{n+1}\right)$, and set $G^{\prime}=G_{n+1}=\left\langle S^{\prime}, \vartheta_{n}^{\prime}\right\rangle \simeq C S p_{2 n+2}(p)$. Now we can embed $W$ into $W^{\prime}, S=S_{n}$ into $S^{\prime}, G=G_{n}$ into $G^{\prime}$ by means of setting $W=\left\langle e_{1}, \ldots, e_{n}, f_{1}, \ldots, f_{n}\right\rangle_{\mathbb{F}_{p}}$, $S=S t_{S^{\prime}}\left(e_{n+1}, f_{n+1}\right), G=\left\langle S, \vartheta_{n+1}\right\rangle$. Clearly, $G \simeq C S p_{2 n}(p)$, and one can identify $\vartheta_{n+1}$ with $\vartheta_{n}$. Choose an irreducible Weil character $\psi^{\prime}$ of $S^{\prime}$ of degree $\left(p^{n+1}+1\right) / 2$ such that $\left(\left.\psi^{\prime}\right|_{S}, \psi\right)_{S}>0$. Let $\chi^{\prime}$ be the rational irreducible character of $G^{\prime}$ of degree $p^{n+1}+1$ and with kernel $C_{(p-1) / 2}$, and let $V^{\prime}=V_{n+1}$ be an irreducible $\mathbb{Q} G^{\prime}$-module with character $\chi^{\prime}$ (cf. Proposition 2.3). Thus $V^{\prime}$ is a faithful $G_{n+1}^{-}$-module.

We collect several facts from $[\mathrm{SchT}]$ and $\S 3$. For any Lagrangian $L^{\prime}$ in $W^{\prime}$ set

$$
G^{\prime}\left(L^{\prime}\right)=\left\{\varphi \in G^{\prime} \mid \varphi\left(L^{\prime}\right)=L^{\prime}\right\}, S^{\prime}\left(L^{\prime}\right)=\left\{\varphi \in G^{\prime}\left(L^{\prime}\right) \mid \operatorname{det}\left(\left.\varphi\right|_{L^{\prime}}\right) \in \mathbb{F}_{p}^{\bullet 2}\right\} .
$$

The subspace $\left\{v \in V^{\prime} \mid \forall \varphi \in S^{\prime}\left(L^{\prime}\right), \varphi(v)=v\right\}$ has dimension 1. Therefore, one can choose an $S^{\prime}\left(L^{\prime}\right)$-stable vector $v\left(L^{\prime}\right)$ such that $\left(v\left(L^{\prime}\right), v\left(L^{\prime}\right)\right)=p^{n / 2}$. Then the lattice

$$
\Delta^{\prime}=\Delta(p, n+1)=\left\langle v\left(L^{\prime}\right) \mid L^{\prime} \in \mathcal{L}\left(W^{\prime}\right)\right\rangle_{\mathbb{Z}}
$$

is an odd unimodular $G^{\prime}$-invariant lattice in $V^{\prime}$. Moreover, $\Delta^{\prime}$ has a $\mathbb{Z}$-linear endomorphism $\sigma$ with the following properties:

(a) $\sigma$ commutes with $S^{\prime}$, and $\sigma \vartheta_{n+1}=-\vartheta_{n+1} \sigma$; 
(b) $\sigma^{2}(v)=\epsilon p v,(\sigma(u), v)=\epsilon(u, \sigma(v)),(\sigma(u), \sigma(v))=p(u, v)$ for any $u, v \in V^{\prime}$, where $\epsilon=(-1)^{(p-1) / 2}$.

Let $L^{\prime}, M^{\prime}$ are any Lagrangians in $W^{\prime}$, and set $u\left(L^{\prime}\right)=\sigma\left(v\left(L^{\prime}\right)\right)$. If $k=$ $\operatorname{dim}_{\mathbb{F}_{p}}\left(L^{\prime} \cap M^{\prime}\right)$ is odd, then $a_{k}=\left|\left(v\left(L^{\prime}\right), v\left(M^{\prime}\right)\right)\right|=p^{(k-1) / 2}, b_{k}=\left|\left(u\left(L^{\prime}\right), u\left(M^{\prime}\right)\right)\right|$ $=p^{(k+1) / 2}, c_{k}=\left|\left(u\left(L^{\prime}\right), v\left(M^{\prime}\right)\right)\right|=0$. If $k$ is even, then $a_{k}=b_{k}=0$, and $c_{k}=p^{k / 2}$.

The descent from $G_{n+1}^{-}$to $G_{n}^{+}$is provided by the following statement. Denote $U=\left\langle e_{n+1}\right\rangle_{\mathbb{F}_{p}}, W^{\prime \prime}=\left\langle e_{n+1}, f_{n+1}\right\rangle_{\mathbb{F}_{p}}$.

Proposition 4.1. The subspace $V=\left\langle v\left(L^{\prime}\right) \mid L^{\prime}=L \oplus U, L \in \mathcal{L}(W)\right\rangle_{\mathbb{Q}}$ of $V^{\prime}$ is a faithful absolutely irreducible $\mathbb{Q} G_{n}^{+}$-module of dimension $p^{n}+1$. Moreover, $V$ is $\sigma$-stable.

Proof. 1) We introduce the following subgroups in $S^{\prime}: B=S t_{S^{\prime}}(W), S^{\prime \prime}=$ $\left\{\varphi \in B \mid \varphi_{W}=1_{W}\right\}, C=S^{\prime \prime} \cap S t_{S^{\prime}}(U), K=S \times C$. Then $S^{\prime \prime} \simeq S p\left(W^{\prime \prime}\right)=S p_{2}(p)$, $C \simeq C_{p} \cdot G L_{1}(p), B=S \times S^{\prime \prime}$. By our definition, $G=\left\langle S, \vartheta_{n+1}\right\rangle$. We also set $H=\left\langle K, \vartheta_{n+1}\right\rangle=G \cdot C$. It is well known that $\left.\psi^{\prime}\right|_{B}=\psi \otimes \psi^{\prime \prime}+\tau \otimes \tau^{\prime \prime}$, where $\psi^{\prime \prime}$ (resp. $\left.\tau^{\prime \prime}\right)$ is an irreducible Weil character of $S^{\prime \prime}$ of degree $(p+1) / 2($ resp. $(p-1) / 2)$. Furthermore, $\tau$ is an irreducible Weil character of $S$ of degree $\left(p^{n}-1\right) / 2\left(\psi_{1}\right.$ in the notation of $\S 1)$. It is easy to check that $\alpha:=\left.\tau^{\prime \prime}\right|_{C} \in \operatorname{Irr}(C)$, and $\left.\psi^{\prime \prime}\right|_{C}=\delta+\beta$, where $\beta \in \operatorname{Irr}(C)$ and $\delta(\varphi)=\left(\frac{\operatorname{det}\left(\left.\varphi\right|_{U}\right)}{p}\right)$ for $\varphi \in C . \quad(\operatorname{In}$ particular, $\delta(1)=1$. Observe that $\beta \neq \delta$. It is so if $p>3$, since in this case $\beta(1)=(p-1) / 2>1$. If $p=3$, then $\mathbb{Q}(\beta)=\mathbb{Q}(\sqrt{-3}) \neq \mathbb{Q}=\mathbb{Q}(\delta)$. Thus

$$
\left.\psi^{\prime}\right|_{K}=\psi \otimes \delta+\psi \otimes \beta+\tau \otimes \alpha
$$

is a sum of three (pairwise distinct) irreducible constituents. From this it follows that

$$
\left.\chi\right|_{K}=(\psi+\bar{\psi}) \otimes \delta+(\psi \otimes \beta+\bar{\psi} \otimes \bar{\beta})+(\tau \otimes \alpha+\bar{\tau} \otimes \bar{\alpha}) .
$$

Observe that $\vartheta_{n+1}$ acts on $S$ as an outer automorphism, and $\vartheta_{n+1}$ interchanges the characters $\psi$ and $\bar{\psi}$. Furthermore, $C \triangleleft H$. Consequently, $\left.\chi\right|_{H}$ has a unique irreducible constituent, say $\gamma$, in which $C$ acts via a multiple of the character $\delta$. This constituent $\gamma$ affords $K$-character $(\psi+\bar{\psi}) \otimes \delta$.

2) Next we observe that $H$ leaves $V$ fixed. Moreover, let $L^{\prime}=L \oplus U$, let $L$ be a Lagrangian in $W$ and $\varphi \in C$. Then $\varphi(L)=L$. Hence, due to (6) the subgroup $C$ acts on $V$ as scalars (and the corresponding character is $\operatorname{dim}_{\mathbb{Q}} V \cdot \delta$ ). By the result of 1 ), $\operatorname{dim}_{\mathbb{Q}} V=p^{n}+1$. Recall that we chose $G \simeq C S p(W)$ to be generated by $S=S p(W)$ and $\vartheta_{n+1}$. We want to find the kernel of $G$ acting on $V$. For consider a generating element $z=\theta E_{2 n}$ of the center $C_{p-1}$ of $C S p(W)$. Then $z$ acting on $W^{\prime}$ has the following matrix: $\operatorname{diag}\left(\theta E_{2 n}, 1, \theta^{2}\right)$ in the basis $\left(e_{1}, \ldots, e_{n}, f_{1}, \ldots, f_{n}, e_{n+1}, f_{n+1}\right)$. If $L^{\prime}=L \oplus U$ ( $L$ any Lagrangian in $W$ ), then due to $(6) z\left(v\left(L^{\prime}\right)\right)=v\left(L^{\prime}\right)$, as $n$ is even. Thus $z$ acts trivially on $V$. We have shown that $V$ is in fact acted on by $C S p_{2 n}(p) / C_{p-1}=G_{n}^{+}$, and this action affords $G_{n}^{+}$-character $\chi^{+}$(cf. Proposition $2.3)$.

3) Finally, we show that $\sigma(V)=V$. Recall that the endomorphism $\sigma$ centralizes $S^{\prime}$. In particular, $\sigma$ centralizes $K$. Hence, the subspace $\sigma(V)$ affords the same $K$ character as of $V$. Since $\vartheta_{n+1}(V)=V$ and $\vartheta_{n+1} \sigma=-\sigma \vartheta_{n+1}, \sigma(V)$ is $\vartheta_{n+1}$-stable, that is, $\sigma(V)$ is an $H$-module. By the results of 1$), \sigma(V)$ also affords the $H$-character $\gamma$. As $\gamma$ is irreducible and it enters $\left.\chi^{\prime}\right|_{H}$ with multiplicity $1, \sigma(V)=V$.

Now we are in a position to give some more explicit lattice constructions. We start with $\left(p^{n}+1\right) / 2$-dimensional lattices. Let $R=S p_{2 m}(q)$, where $q=p^{2 f}$. Then 
$R$ has two irreducible Weil characters $\varrho, \varrho^{*}$ of degree $\left(q^{m}+1\right) / 2$. These characters are conjugate under some outer automorphism of $R$. Both of them are rational, as shown in [Gro]. We want to expose an explicit construction for $\mathbb{Z} R$-lattices of dimension $\left(q^{m}+1\right) / 2$. To this end, put $n=m f$. Consider the natural $\mathbb{F}_{q} R$ module $W^{(f)}=\mathbb{F}_{q}^{2 m}$ endowed with a non-degenerate $\mathbb{F}_{q}$-valued symplectic form $\langle\cdot, \cdot\rangle_{(f)}$. Then we can identify $W$ with $W^{(f)}$ viewed as $\mathbb{F}_{p}$-space and assume that $\langle u, v\rangle=\operatorname{Tr}_{\mathbb{F}_{q} / \mathbb{F}_{p}}\langle u, v\rangle_{(f)}$. This identification embeds $R=S p\left(W^{(f)}\right)$ canonically in $S_{n}=S p(W)$. One may also suppose that $\varrho=\left.\psi\right|_{R}$. Clearly, any Lagrangian

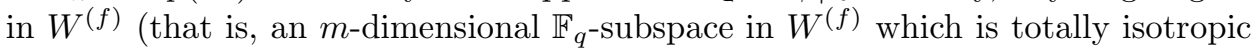
w.r.t. $\left.\langle\cdot, \cdot\rangle_{(f)}\right)$ is also a Lagrangian in $W$. We call these special Lagrangians $\mathbb{F}_{q^{-}}$ Lagrangians in $W$.

Theorem 4.2. Keep the above notation. Set

$$
\left.\Delta(q, m)=\left\langle v\left(L^{\prime}\right)\right| L^{\prime}=L \oplus U, L \text { any } \mathbb{F}_{q} \text {-Lagrangian in } W\right\rangle_{\mathbb{Z}} \text {. }
$$

Then $\Delta(q, m)$ is an R-invariant integral lattice affording the Weil character $\varrho$.

Proof. In addition to $\Gamma:=\Delta(q, m)$ we consider

$$
\left.\Gamma^{\prime}=\left\langle u\left(L^{\prime}\right)\right| L^{\prime}=L \oplus U, L \text { an } \mathbb{F}_{q^{-L a g r a n g i a n}} \text { in } W\right\rangle_{\mathbb{Z}} .
$$

Clearly, $\Gamma$ and $\Gamma^{\prime}$ are invariant under $R$. We have mentioned that the restriction $\left.\psi\right|_{R}$ is equal to $\varrho$ and so it is irreducible. Hence $\left.\chi\right|_{R}=2 \varrho$. From this it follows that $\operatorname{dim}_{\mathbb{Z}} \Gamma$ and $\operatorname{dim}_{\mathbb{Z}} \Gamma^{\prime}$ are at least $\varrho(1)=\left(p^{n}+1\right) / 2$. Observe that $\Gamma \perp \Gamma^{\prime}$. For,

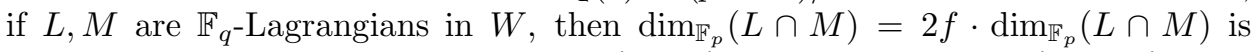
always even. This implies that $\operatorname{dim}_{\mathbb{F}_{p}}\left(L^{\prime} \cap M^{\prime}\right)$ is odd, and so $\left(v\left(L^{\prime}\right), u\left(M^{\prime}\right)\right)=0$ by Lemma 3.3. By Proposition 4.1, $\Gamma$ and $\Gamma^{\prime}$ are contained in the $\mathbb{Q}$-space $V$ of dimension $p^{n}+1$, and the scalar product on $V$ is positive definite. Hence we must have $\operatorname{dim}_{\mathbb{Z}} \Gamma=\varrho(1)$, and $\Gamma=\Delta(q, m)$ is an $R$-invariant integral lattice affording the Weil character $\varrho$.

Corollary 4.3. In the notation of Proposition 4.1, $\operatorname{det} V=p \mathbb{Q}^{\bullet 2}$. On the other hand, if $p \equiv 1 \bmod 4$ and $V$ is considered as a $G_{n}^{-}$-module by means of Proposition 2.4, then $\operatorname{det} V=\mathbb{Q}^{\bullet 2}$.

Proof. The proof of Theorem 4.2 shows that $V$ contains the lattice $\Gamma \oplus \sigma(\Gamma)$ of determinant $\operatorname{det} \Gamma \cdot \operatorname{det} \sigma(\Gamma)=p^{\left(p^{n}+1\right) / 2}(\operatorname{det} \Gamma)^{2} \in p \mathbb{Q}^{\bullet 2}$. The other claim follows from the oddness of $\left(p^{n}+1\right) / 2$.

Theorem 4.4. Keep the above notation. Set

$$
\Delta=\Delta(p, n)=\left\langle v\left(L^{\prime}\right) \mid L^{\prime}=L \oplus U, L \in \mathcal{L}(W)\right\rangle_{\mathbb{Z}}
$$

Then $\Delta$ is a $G_{n}^{+}$-invariant p-modular lattice.

Proof. Recall that the scalar product on $V$ is inherited from the one on $V^{\prime}$, and the dual $\Delta^{\#}$ to $\Delta$ is taken under this scalar product. Clearly, $\Delta$ is fixed by $G_{n}^{+}$. Applying Proposition 3.8, we see that $u\left(L^{\prime}\right)=\sigma\left(v\left(L^{\prime}\right)\right)$ is contained in $\Delta$ for any $L$, and $\Delta$ is $\sigma$-stable.

1) First assume that $\operatorname{det} \Delta$ is divisible by some prime $r \neq 2, p$. Consider the form $(\bar{x}, \bar{y})_{r}=(x, y) \bmod r$ on $\Delta / r \Delta$, where $\bar{x}=x+r \Delta, \bar{y}=y+r \Delta$. As $r$ divides $\operatorname{det} \Delta$, this $G$-invariant symmetric bilinear form is degenerate, and so its kernel $\left(\Delta \cap r \Delta^{\#}\right) / r \Delta$ is nonzero, i.e. $\Delta \supseteq \Delta \cap r \Delta^{\#} \supset r \Delta$. By Proposition 2.2 (i), this means $\Delta=\Delta \cap r \Delta^{\#}$. Hence $(u, v) \in r \mathbb{Z}$ for any $u, v \in \Delta$. In the meantime, 
$\left(v\left(L^{\prime}\right), v\left(M^{\prime}\right)\right)= \pm 1$ for $L^{\prime}=L \oplus U, M^{\prime}=M \oplus U$ with $\operatorname{dim}(L \cap M)=0$, a contradiction.

2) At this point we show that $\operatorname{det} \Delta$ is odd. Suppose the contrary: 2 divides $\operatorname{det} \Delta$. Consider the form $(\bar{x}, \bar{y})_{2}=(x, y) \bmod 2$ on $\Delta / 2 \Delta$, where $\bar{x}=x+2 \Delta$, $\bar{y}=y+2 \Delta$. As $2 \mid \operatorname{det} \Delta, \Delta^{\prime}=: \Delta \cap 2 \Delta^{\#}$ contains properly $2 \Delta$. Since $\Delta$ is an odd lattice, its even part $\Delta^{0}=\{v \in \Delta \mid(v, v) \in 2 \mathbb{Z}\}$ is a sublattice of index 2 in $\Delta$. Moreover, $\Delta^{0} \supset \Delta^{\prime}$. (For $\Delta^{\prime}$ is clearly contained in $\Delta^{0}$. On the other hand,

$$
\left(v\left(L^{\prime}\right)+u\left(L^{\prime}\right), v\left(L^{\prime}\right)+u\left(L^{\prime}\right)\right) \in 2 \mathbb{Z},\left(v\left(L^{\prime}\right)+u\left(L^{\prime}\right), v\left(L^{\prime}\right)\right)=p^{n / 2} \notin 2 \mathbb{Z},
$$

i.e. $v\left(L^{\prime}\right)+u\left(L^{\prime}\right) \in \Delta^{0} \backslash \Delta^{\prime}$.) Applying Proposition 2.2 (ii), we see that $A \supset B \supset$ $C \supset 0$ is a composition series for the $\mathbb{F}_{2} G$-module $A=\Delta / 2 \Delta$, where $B=\Delta^{0} / 2 \Delta$, $C=\Delta^{\prime} / 2 \Delta$.

We exhibit one more nonzero proper submodule inside $B$. Set $\Gamma=\langle v+\sigma(v)|$ $v \in \Delta\rangle_{\mathbb{Z}}+2 \Delta$. Since $g \sigma= \pm \sigma g$ for all $g \in G, \Gamma$ is $G$-stable. Furthermore,

$$
(u+\sigma(u), v+\sigma(v))=(p+1)(u, v)+(1+\epsilon)(u, \sigma(v)) \in 2 \mathbb{Z}
$$

due to the properties of the endomorphism $\sigma$. Thus $D=\Gamma / 2 \Delta$ is a $G$-submodule of $B$ and $D$ is totally isotropic w.r.t. $(\cdot, \cdot)_{2}$. Since $\left(v\left(L^{\prime}\right)+u\left(L^{\prime}\right), v\left(L^{\prime}\right)\right)=p^{n / 2}$, we see that $v\left(L^{\prime}\right)+u\left(L^{\prime}\right) \in \Gamma \backslash \Delta^{\prime}$. From this it follows that $0 \neq D \neq C$. Since $B \supset C \supset 0$ is a composition series for $B$, we must have $B=C+D$. But $C=\operatorname{Ker}(\cdot, \cdot)_{2}$; hence we come to the conclusion that $B$ is totally isotropic w.r.t. $(\cdot, \cdot)_{2}$. On the other hand, choosing

$$
\begin{gathered}
L_{1}=\left\langle e_{1}, \ldots, e_{n}, e_{n+1}\right\rangle_{\mathbb{F}_{p}}, L_{2}=\left\langle f_{1}, \ldots, f_{n}, e_{n+1}\right\rangle_{\mathbb{F}_{p}}, \\
L_{3}=\left\langle e_{1}, \ldots, e_{n-2}, e_{n-1}+f_{n-1}, f_{n}, e_{n+1}\right\rangle_{\mathbb{F}_{p}},
\end{gathered}
$$

we get $v\left(L_{1}\right)+v\left(L_{2}\right), v\left(L_{1}\right)+v\left(L_{3}\right) \in \Delta^{0}$ with

$$
\left(v\left(L_{1}\right)+v\left(L_{2}\right), v\left(L_{1}\right)+v\left(L_{3}\right)\right) \equiv p^{n / 2}+p^{(n-2) / 2}+1+0 \equiv 1 \bmod 2,
$$

a contradiction.

3) Observe that $p \Delta^{\#} \supseteq \sigma(\Delta)$. (Indeed, $\Delta$ is generated by the vectors $v\left(L^{\prime}\right)$, and $\sigma(\Delta)$ is generated by the vectors $u\left(M^{\prime}\right)$, with $L^{\prime}=L \oplus U, M^{\prime}=M \oplus U$, $L, M$ arbitrary Lagrangians in $W$. It is obvious that $k=\operatorname{dim}\left(L^{\prime} \cap M^{\prime}\right) \geq 1$. But $\left|\left(v\left(L^{\prime}\right), u\left(M^{\prime}\right)\right)\right|=c_{k}$ is 0 if $k$ is odd, and $p^{k / 2}$ if $k$ is even. Hence $c_{k}$ is divisible by p.) In fact we have

$$
\Delta \cap p \Delta^{\#}=\sigma(\Delta) .
$$

For, assume the contrary. Then $\Delta \supseteq \Delta \cap p \Delta^{\#} \supset \sigma(\Delta) \supset p \Delta$. By Proposition 2.2 (iii) $\Delta / \sigma(\Delta)$ is an irreducible $\mathbb{F}_{p} G$-module. Hence $\Delta \cap p \Delta^{\#}=\Delta, \Delta \subseteq p \Delta^{\#}$. The last inclusion contradicts the equality $\left(v\left(L^{\prime}\right), v\left(M^{\prime}\right)\right)= \pm 1$ for $\operatorname{dim}\left(L^{\prime} \cap M^{\prime}\right)=1$.

In addition to (10) we show that

$$
\Delta \cap p^{2} \Delta^{\#} \subseteq p \Delta .
$$

To this end we denote $\Lambda=\Delta \cap p \Delta^{\#}$. Then $\Lambda \cap p^{2} \Lambda^{\#}$ is a proper sublattice of $\Lambda$, because $u\left(L^{\prime}\right), u\left(M^{\prime}\right) \in \Lambda$ and $\left(u\left(L^{\prime}\right), u\left(M^{\prime}\right)\right)= \pm p$ provided that $\operatorname{dim}\left(L^{\prime} \cap M^{\prime}\right)=1$. Furthermore, $\left(\Delta \cap p \Delta^{\#}, p \Delta\right) \subseteq p^{2} \mathbb{Z}$. Thus we have

$$
p \Delta \subseteq \Lambda \cap p^{2} \Lambda^{\#} \subset \Lambda=\sigma(\Delta) .
$$


Now the irreducibility of the $\mathbb{F}_{p} G$-module $\sigma(\Delta) / p \Delta$ implies that $p \Delta=\Lambda \cap p^{2} \Lambda^{\#}$. Keeping in mind that

$$
\Lambda \cap p^{2} \Lambda^{\#}=\left(\Delta \cap p \Delta^{\#}\right) \cap\left(p \Delta+p^{2} \Delta^{\#}\right) \supseteq \Delta \cap p^{2} \Delta^{\#},
$$

one obtains (11).

4) By the results of 1) and 2), $\operatorname{det} \Delta$ is not divisible by any prime $r$ other than $p$. Hence det $\Delta=p^{m}$ and so $\Delta \supseteq p^{m} \Delta^{\#}$ for some non-negative integer $m$. Choose the minimal non-negative integer $\ell$ such that $\Delta \supseteq p^{\ell} \Delta^{\#}$. If $\ell=0$, then by (10) one has $\sigma(\Delta)=p \Delta$, a contradiction. Assume that $\ell \geq 2$. Then applying (11) we have

$$
p^{\ell} \Delta^{\#} \subseteq \Delta \cap p^{2} \Delta^{\#} \subseteq p \Delta,
$$

i.e. $p^{\ell-1} \Delta^{\#} \subseteq \Delta$, contrary to the choice of $\ell$. Hence $\ell=1$. In this case (10) yields $p \Delta^{\#}=\sigma(\Delta), \Delta=\sigma\left(\Delta^{\#}\right)$. In other words, $\Delta$ is $p$-modular.

From now on, when considering $\Delta(p, n)$ with $n$ even, we denote $v(L \oplus U)$ by $v(L)$ ( $L$ a Lagrangian in $W$ ) and then forget the initial descent $n+1 \leadsto n$. In particular, $(v(L), v(M))= \pm p^{k / 2}$ if $k=\operatorname{dim}(L \cap M)$ is even, and 0 otherwise. The signs involved in this formula will be determined in the next section.

\section{Maslov indeX AND Gram matriX}

Let $k$ be any field of characteristic other than 2 and $S(k)=S p_{2 n}(k)$. If $k=\mathbb{C}$ or $k$ is a finite field (and $(n,|k|) \neq(1,9)$ ), then it is well known that $S(k)$ is simply connected. However, if $k$ is $\mathbb{R}$ or any local field, then $S(k)$ is not simply connected, and $S(k)$ has a double covering group called the metasymplectic group. An important role in physics is played by a faithful complex representation of the metasymplectic group called the Shale-Weil representation. A key ingredient of constructing this representation is Maslov index (or Maslov-Kashiwara index), which is defined on triples of Lagrangians inside the symplectic space $k^{2 n}$. For more detail the reader is referred to $[\mathrm{LiV}]$.

Remarkably, we can define a discrete analogue of Maslov index for $S p_{2 n}(p)$, which enables one to completely determine the Gram matrices of the lattices $\Delta(p, n), n$ any integer and $p$ any odd prime (cf. Theorems $3.9,4.4)$, and the lattices $\Delta^{-}(p, n)$ (in the case $p \equiv 1 \bmod 4)$. Here, $\Delta^{-}(p, n)$ is obtained from $\Delta(p, n)$ by means of Proposition 2.4 (with $G^{+}=G_{n}^{-}$if $n$ is odd and $G^{+}=C_{2} \times G_{n}^{+}$if $n$ is even). Throughout this section, Lagrangians are considered oriented.

First we deal with the lattices $\Delta(p, n)$. Fix an oriented Lagrangian $L_{0}$ with an ordered basis $\left(u_{1}, \ldots, u_{n}\right)$ (for short: $L_{0}=\left(u_{1}, \ldots, u_{n}\right)$ ), and a generating vector $v\left(L_{0}\right)$ of $\Delta\left(L_{0}\right)$. For an arbitrary oriented Lagrangian $M=\left(v_{1}, \ldots, v_{n}\right)$ we find an element $\nu_{M} \in S p_{2 n}(p)$ such that $\nu_{M}\left(u_{i}\right)=v_{i}$ for all $i$, and set $v(M)=\nu_{M}\left(v\left(L_{0}\right)\right)$. It is easy to see that this definition does not depend on the choice of $\nu_{M}$. Finally, we put $u(L)=\sigma(v(L))$ (cf. Lemma 3.6).

Definition 5.1. Let $p$ be any odd prime and $n$ any integer. Let $L, M$ be arbitrary oriented Lagrangians in $W=\mathbb{F}_{p}^{2 n}$. Then the index $[L, M]$ of the ordered pair $(L, M)$ is defined to be $\left(\frac{\operatorname{det} F}{p}\right)$, where the matrix $F$ is defined as follows. Let $\operatorname{dim}(L \cap M)=k$, choose ordered bases

$$
\left(u_{1}, \ldots, u_{k}, v_{1}, \ldots, v_{n-k}\right),\left(u_{1}, \ldots, u_{k}, w_{1}, \ldots, w_{n-k}\right)
$$

of $L, M$, respectively; set $F:=F(L, M):=\left(\left\langle v_{i}, w_{j}\right\rangle\right)_{1 \leq i, j \leq n-k}$. 
Proposition 5.2. The index is well defined. It is symmetric and $G_{n}$-invariant on pairs $(L, M)$ with $n-\operatorname{dim}(L \cap M)$ even. Moreover, if $L, M, L^{\prime}, M^{\prime}$ are oriented Lagrangians and $\operatorname{dim}(L \cap M)=\operatorname{dim}\left(L^{\prime} \cap M^{\prime}\right)$, then

$$
\begin{aligned}
& {[L, M](v(L), v(M))=\left[L^{\prime}, M^{\prime}\right]\left(v\left(L^{\prime}\right), v\left(M^{\prime}\right)\right),} \\
& {[L, M](u(L), v(M))=\left[L^{\prime}, M^{\prime}\right]\left(u\left(L^{\prime}\right), v\left(M^{\prime}\right)\right),} \\
& {[L, M](u(L), u(M))=\left[L^{\prime}, M^{\prime}\right]\left(u\left(L^{\prime}\right), u\left(M^{\prime}\right)\right) .}
\end{aligned}
$$

Proof. First we show that $\left(\frac{\operatorname{det} F}{p}\right)$ is independent of the bases chosen. For, suppose

$$
\left(u_{1}^{\prime}, \ldots, u_{k}^{\prime}, v_{1}^{\prime}, \ldots, v_{n-k}^{\prime}\right),\left(u_{1}^{\prime}, \ldots, u_{k}^{\prime}, w_{1}^{\prime}, \ldots, w_{n-k}^{\prime}\right)
$$

are other ordered bases of

$$
L=\left(u_{1}, \ldots, u_{k}, v_{1}, \ldots, v_{n-k}\right), M=\left(u_{1}, \ldots, u_{k}, w_{1}, \ldots, w_{n-k}\right) .
$$

Then the transition matrices (from the old bases to the new bases) are $\left(\begin{array}{cc}A & X \\ 0 & B\end{array}\right)$ and $\left(\begin{array}{cc}A & Y \\ 0 & C\end{array}\right)$, where $A \in G L_{k}(p), X, Y \in M_{k, n-k}\left(\mathbb{F}_{p}\right), B, C \in G L_{n-k}(p)$. Since $L, M$ are oriented, $\operatorname{det} A \cdot \operatorname{det} B$ and $\operatorname{det} A \cdot \operatorname{det} C$ belong to $\mathbb{F}_{p}^{\bullet 2}$. Clearly, $F$ is changed to ${ }^{t} B F C$ and $\left(\frac{\operatorname{det} F}{p}\right)=\left(\frac{\operatorname{det}^{t} B F C}{p}\right)$.

If $g \in G_{n}$ and $\langle g u, g v\rangle=\lambda \cdot\langle u, v\rangle$ for all $u, v \in V$, then $[g(L), g(M)]=$ $\left(\frac{\lambda}{p}\right)^{n-k}[L, M]$. Furthermore, $[M, L]=\epsilon^{n-k}[L, M]$. In particular, $[L, M]$ is symmetric and $G_{n}$-invariant on pairs $(L, M)$ with $n-\operatorname{dim}(L \cap M)$ even.

Finally, assume

$$
\begin{aligned}
& L=\left(u_{1}, \ldots, u_{k}, v_{1}, \ldots, v_{n-k}\right), \quad M=\left(u_{1}, \ldots, u_{k}, w_{1}, \ldots, w_{n-k}\right), \\
& L^{\prime}=\left(u_{1}^{\prime}, \ldots, u_{k}^{\prime}, v_{1}^{\prime}, \ldots, v_{n-k}^{\prime}\right), M^{\prime}=\left(u_{1}^{\prime}, \ldots, u_{k}^{\prime}, w_{1}^{\prime}, \ldots, w_{n-k}^{\prime}\right)
\end{aligned}
$$

are oriented Lagrangians in $W$. Then there exists $g \in S p_{2 n}(p)$ such that $g(L)= \pm L^{\prime}, g(M)= \pm M^{\prime}$. Let $\left(\begin{array}{cc}A & X \\ 0 & B\end{array}\right)\left(\operatorname{resp}\right.$. $\left.\left(\begin{array}{cc}A & Y \\ 0 & C\end{array}\right)\right)$ be the transition matrix from the basis $\left(g\left(u_{1}\right), \ldots, g\left(u_{k}\right), g\left(v_{1}\right), \ldots, g\left(v_{n-k}\right)\right)$ of $g(L)$ to the basis $\left(u_{1}^{\prime}, \ldots, u_{k}^{\prime}, v_{1}^{\prime}, \ldots, v_{n-k}^{\prime}\right)$ of $L^{\prime}$ (resp. from the basis $\left(g\left(u_{1}\right), \ldots, g\left(u_{k}\right), g\left(w_{1}\right), \ldots\right.$, $\left.g\left(w_{n-k}\right)\right)$ to $\left.\left(u_{1}^{\prime}, \ldots, u_{k}^{\prime}, w_{1}^{\prime}, \ldots, w_{n-k}^{\prime}\right)\right)$. Then

$$
v\left(L^{\prime}\right)=\left(\frac{\operatorname{det} A \cdot \operatorname{det} B}{p}\right) g(v(L)), v\left(M^{\prime}\right)=\left(\frac{\operatorname{det} A \cdot \operatorname{det} C}{p}\right) g(v(M))
$$

and so $\left(v\left(L^{\prime}\right), v\left(M^{\prime}\right)\right)=\left(\frac{\operatorname{det} B \cdot \operatorname{det} C}{p}\right)(v(L), v(M))$. On the other hand, one can show that $F\left(L^{\prime}, M^{\prime}\right)={ }^{t} B \cdot F(L, M) \cdot C$, yielding $\left[L^{\prime}, M^{\prime}\right]=\left(\frac{\operatorname{det} B \cdot \operatorname{det} C}{p}\right)[L, M]$. Hence $[L, M](v(L), v(M))=\left[L^{\prime}, M^{\prime}\right]\left(v\left(L^{\prime}\right), v\left(M^{\prime}\right)\right)$. The identities

$$
\begin{aligned}
& {[L, M](u(L), v(M))=\left[L^{\prime}, M^{\prime}\right]\left(u\left(L^{\prime}\right), v\left(M^{\prime}\right)\right),} \\
& {[L, M](u(L), u(M))=\left[L^{\prime}, M^{\prime}\right]\left(u\left(L^{\prime}\right), u\left(M^{\prime}\right)\right)}
\end{aligned}
$$

are proved in the same way.

Theorem 5.3. Let $p$ be any odd prime and $n$ any integer, and let $\epsilon=(-1)^{(p-1) / 2}$. Under the above notation one has

$$
(v(L), v(M))=(\epsilon / p)^{(n-k) / 2} p^{[n / 2]}[L, M]
$$

for any oriented Lagrangians $L, M$ with $k=\operatorname{dim}(L \cap M)$ and $n-k$ even. 
Proof. By Corollary 3.5, Theorem 4.4 and Proposition 5.2, there are constants $C_{k}=$ \pm 1 such that $[L, M](v(L), v(M))=p^{[n / 2]-(n-k) / 2} C_{k}$ for any oriented Lagrangians $L, M$ with $k=\operatorname{dim}(L \cap M)$ and $n-k$ even. We want to show that

$$
C_{k}=\epsilon^{(n-k) / 2} \text {. }
$$

Clearly, (12) holds for $k=n$.

1) At this point we prove (12) for $k=n-2$ (and $n \geq 2$ ). Because of the descent $n \leadsto n-1$ used in Theorem 4.4 , we can restrict ourselves to the case $n$ is odd (and so $n \geq 3$ ). In order to determine $C_{n-2}$, we use the standard spread $\left\{W^{\infty}, W^{\lambda} \mid \lambda \in \mathbb{F}_{q}\right\}$ of $W=\mathbb{F}_{p}^{2 n}$ (see the discussion before (7)). As usual, we assume that $\left\langle e_{i}, f_{j}\right\rangle=\delta_{i, j}$, where $W^{0}=\left(e_{1}, \ldots, e_{n}\right), W^{\infty}=\left(f_{1}, \ldots, f_{n}\right)$. Due to our identification of $W$ with $\mathbb{F}_{q}^{2}, q=p^{n}$, we have $e_{i}=\left(\alpha_{i}, 0\right), f_{i}=\left(0, \beta_{i}\right)$ for any $i$ and some $\alpha_{i}, \beta_{i} \in \mathbb{F}_{q}$. Observe that there is a map from $S p_{2 n}(p)$ which sends the oriented Lagrangian $L_{0}:=W^{0}$ to $W^{\infty}$ (resp. to $W^{\lambda}=\left(\left(\alpha_{1}, \lambda \alpha_{1}\right), \ldots,\left(\alpha_{n}, \lambda \alpha_{n}\right)\right)$, $\left.\lambda \in \mathbb{F}_{q}\right)$. Now take

$$
L=\left(e_{1}, f_{2}, f_{3}, \ldots, f_{n}\right), M=\left(f_{1}, e_{2}, f_{3}, \ldots, f_{n}\right) .
$$

Then $\operatorname{dim}(L \cap M)=n-2,[L, M]=\epsilon . \operatorname{Since} \operatorname{dim}\left(L \cap W^{\infty}\right)=\operatorname{dim}\left(M \cap W^{\infty}\right)=n-1$, we have

$$
v(L)=\sum_{\lambda \in \mathbb{F}_{q}} a_{\lambda} v\left(W^{\lambda}\right), v(M)=\sum_{\lambda \in \mathbb{F}_{q}} b_{\lambda} v\left(W^{\lambda}\right)
$$

and so

$$
\epsilon p^{(n-3) / 2} C_{n-2}=(v(L), v(M))=p^{(n-1) / 2} \sum_{\lambda \in \mathbb{F}_{q}} a_{\lambda} b_{\lambda} .
$$

One easily sees that $a_{\lambda} \neq 0$ if and only if $\operatorname{tr}\left(\lambda\left(\alpha_{1}\right)^{2}\right)=0$. Similarly, $b_{\lambda} \neq 0$ if and only if $\operatorname{tr}\left(\lambda\left(\alpha_{2}\right)^{2}\right)=0$. Observe that $\left(\alpha_{1}\right)^{2}$ and $\left(\alpha_{2}\right)^{2}$ are linearly independent over $\mathbb{F}_{p}$; otherwise $\mathbb{F}_{q}$ would contain $\mathbb{F}_{p}\left(\alpha_{1} / \alpha_{2}\right)=\mathbb{F}_{p^{2}}$, contrary to the assumption that $n$ is odd. Hence $a_{\lambda} b_{\lambda} \neq 0$ for exactly $p^{n-2}$ values of $\lambda \in \mathbb{F}_{q}$. Moreover, if $a_{\lambda} b_{\lambda} \neq 0$, then $a_{\lambda} b_{\lambda}=p^{1-n}$, since in this case $\operatorname{dim}\left(L \cap W^{\lambda}\right)=\operatorname{dim}\left(M \cap W^{\lambda}\right)=1$ and $\left[W^{\lambda}, L\right]=\left[W^{\lambda}, M\right]=1$. Bearing (13) in mind, we obtain $C_{n-2}=\epsilon$, as stated.

2) Here we show that

$$
C_{n-2[n / 2]}=C_{n+2-2[n / 2]} C_{n-2}
$$

for any $n \geq 2$.

2a) Because of the descent $n \leadsto n-1$ used in Theorem 4.4, we can restrict ourselves to the case $n$ is even. In order to prove (14): $C_{0}=C_{2} C_{n-2}$, we consider the standard spread $\left\{W^{\infty}, W^{\lambda} \mid \lambda \in \mathbb{F}_{q}\right\}$ of $W=\mathbb{F}_{p}^{2 n}$. As above, we assume that $\left\langle e_{i}, f_{j}\right\rangle=\delta_{i, j}$, where $W^{0}=\left(e_{1}, \ldots, e_{n}\right), W^{\infty}=\left(f_{1}, \ldots, f_{n}\right)$. Due to our identification of $W$ with $\mathbb{F}_{q}^{2}, q=p^{n}$, we have $e_{i}=\left(\alpha_{i}, 0\right), f_{i}=\left(0, \beta_{i}\right)$ for any $i$ and some $\alpha_{i}, \beta_{i} \in \mathbb{F}_{q}$. Since $n$ is even, without loss of generality we may suppose that $\alpha_{1}=1$ and $\alpha_{2}=e \in \mathbb{F}_{p^{2}} \backslash \mathbb{F}_{p}$. Observe that there is a map from $S p_{2 n}(p)$ which sends the oriented Lagrangian $L_{0}:=W^{0}$ to $W^{\infty}$ (resp. to $W^{\lambda}=\left(\left(\alpha_{1}, \lambda \alpha_{1}\right), \ldots,\left(\alpha_{n}, \lambda \alpha_{n}\right)\right)$, $\left.\lambda \in \mathbb{F}_{q}\right)$. Our identification of $W$ with $\mathbb{F}_{q}^{2}$ embeds $R=S L_{2}(q)$ naturally in $S p_{2 n}(p)$. Consider the following elements

$$
r_{a}=\left(\begin{array}{cc}
a^{-1} & 0 \\
0 & a
\end{array}\right), s_{b}=\left(\begin{array}{cc}
1 & 0 \\
b & 1
\end{array}\right), t=\left(\begin{array}{rr}
0 & -1 \\
1 & 0
\end{array}\right), a \in \mathbb{F}_{q}^{\bullet}, b \in \mathbb{F}_{q}
$$


of $R$. Then they act on the vectors $v\left(W^{\lambda}\right)$ as follows:

$$
\begin{array}{llll}
r_{a}: & v\left(W^{\infty}\right) \mapsto\left(\frac{a}{q}\right) v\left(W^{\infty}\right), & v\left(W^{\lambda}\right) \mapsto\left(\frac{a}{q}\right) v\left(W^{a^{2} \lambda}\right), \\
s_{b}: & v\left(W^{\infty}\right) \mapsto v\left(W^{\infty}\right), & v\left(W^{\lambda}\right) \mapsto v\left(W^{\lambda+b}\right), \\
t: & v\left(W^{\infty}\right) \leftrightarrow \mu v\left(W^{0}\right), & v\left(W^{\lambda}\right) \mapsto\left(\frac{\lambda}{q}\right) v\left(W^{-1 / \lambda}\right) .
\end{array}
$$

Here, $\left(\frac{\lambda}{q}\right)=\lambda^{(q-1) / 2}$ and $\mu=\left(\frac{\operatorname{det} T}{q}\right)$, where $T=\left(\operatorname{tr}\left(\alpha_{i} \beta_{j}\right)\right)_{1 \leq i, j \leq n}$ and $\operatorname{tr}:=$

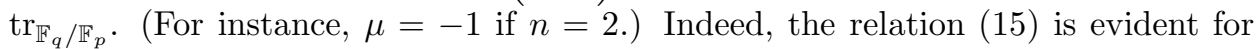
$s_{b}$. Furthermore, the factor $\left(\frac{a}{q}\right)$ appears in (15) for $r_{a}$, since the map sending each $f_{i}$ to $a f_{i}$ has determinant $N_{\mathbb{F}_{q} / \mathbb{F}_{p}}(a)$. Similarly, for any $\lambda \in \mathbb{F}_{q}$, the map sending each $\left(a^{-1} \alpha_{i}, a \lambda \alpha_{i}\right)$ to $\left(\alpha_{i}, a^{2} \lambda \alpha_{i}\right)$ has determinant $N_{\mathbb{F}_{q} / \mathbb{F}_{p}}(a)$. By the same reason the factor $\left(\frac{\lambda}{q}\right)$ appears in the formula for $t$. Finally, $t\left(e_{i}\right)=\left(0, \alpha_{i}\right)$, and the map sending each $\left(0, \alpha_{i}\right)$ to $f_{i}=\left(0, \beta_{i}\right)$ has matrix $T^{-1}$.

2b) Using the action of $s_{b}$, we see that there is $\gamma= \pm 1$ such that $\left(v\left(W^{\infty}\right), v\left(W^{\lambda}\right)\right)$ $=\gamma$ for all $\lambda \in \mathbb{F}_{q}$. Next, $t$ acting on this relation yields $\left(v\left(W^{0}\right), v\left(W^{\lambda}\right)\right)=\mu \gamma$ if $\lambda \in \mathbb{F}_{q}$ is a square, and $-\mu \gamma$ otherwise. Finally, using the action of $s_{b}$ once more, we see that for $\lambda \neq \lambda^{\prime} \in \mathbb{F}_{q},\left(v\left(W^{\lambda}\right), v\left(W^{\lambda^{\prime}}\right)\right)=\mu \gamma$ if $\lambda-\lambda^{\prime}$ is a square, and $-\mu \gamma$ otherwise. Since $\left[W^{\infty}, W^{0}\right]=1, \gamma=C_{0}$.

2c) The proof of Theorem 4.2 shows that $v\left(W^{\infty}\right)$ and $v\left(W^{\lambda}\right), \lambda \in \mathbb{F}_{q}$, are linearly independent: $v\left(W^{\infty}\right)=\sum_{\lambda \in \mathbb{F}_{q}} a_{\lambda} v\left(W^{\lambda}\right)$ for $a_{\lambda} \in \mathbb{C}$. Averaging this relation by means of $s_{b}, b \in \mathbb{F}_{q}$, we get $v\left(W^{\infty}\right)=a \sum_{\lambda \in \mathbb{F}_{q}} v\left(W^{\lambda}\right)$ for $a \in \mathbb{C}$. Hence

$$
\begin{aligned}
\gamma & =\left(v\left(W^{\infty}\right), v\left(W^{0}\right)\right) \\
& =a \sqrt{q}+a \sum_{\lambda \in \mathbb{F}_{q}^{\bullet 2}}\left(v\left(W^{0}\right), v\left(W^{\lambda}\right)\right)+a \sum_{\lambda \in \mathbb{F}_{q}^{\bullet} \backslash \mathbb{F}_{q}^{\bullet 2}}\left(v\left(W^{0}\right), v\left(W^{\lambda}\right)\right) \\
& =a \sqrt{q}+\frac{q-1}{2} a \mu \gamma-\frac{q-1}{2} a \mu \gamma=a \sqrt{q},
\end{aligned}
$$

i.e. $a=\gamma p^{-n / 2}$. We have shown that

$$
v\left(W^{\infty}\right)=C_{0} p^{-n / 2} \sum_{\lambda \in \mathbb{F}_{q}} v\left(W^{\lambda}\right) .
$$

2d) Now we consider the oriented Lagrangian $M=\left(e_{1}, e_{2}, f_{3}, \ldots, f_{n}\right)$. Since $\operatorname{dim}\left(M \cap W^{\infty}\right)=n-2$ and $\left[M, W^{\infty}\right]=1,\left(v(M), v\left(W^{\infty}\right)\right)=p^{n / 2-1} C_{n-2}$. We compute this scalar product in another way using (16). For $\lambda \in \mathbb{F}_{q}$, it is clear that $\operatorname{dim}\left(M \cap W^{\lambda}\right) \leq 2$. Moreover, $\operatorname{dim}\left(M \cap W^{\lambda}\right)=2$ if and only if

$$
\operatorname{tr}(\lambda)=\operatorname{tr}(\lambda e)=\operatorname{tr}\left(\lambda e^{2}\right)=0 .
$$

(Recall that we have chosen $\alpha_{1}=1$ and $\alpha_{2}=e \in \mathbb{F}_{p^{2}} \backslash \mathbb{F}_{p}$.) Since $\mathbb{F}_{p}(e)=\mathbb{F}_{p^{2}},(17)$ holds for exactly $p^{n-2}$ values of $\lambda \in \mathbb{F}_{q}$. Denote $\mathcal{X}=\left\{\lambda \in \mathbb{F}_{q} \mid \operatorname{dim}\left(M \cap W^{\lambda}\right)=2\right\}$, $\mathcal{Y}=\mathbb{F}_{q} \backslash \mathcal{X}$. By the choice of $\alpha_{1}$ and $\alpha_{2}, M \cap W^{\lambda}=0$ for any $\lambda \in \mathcal{Y}$.

2e) It is easy to check that $\left[M, W^{\lambda}\right]=1$ for any $\lambda \in \mathcal{X}$. In particular,

$$
\sum_{\lambda \in \mathcal{X}}\left(v(M), v\left(W^{\lambda}\right)\right)=p^{n-1} C_{2}
$$

Similarly, $\left[M, W^{\lambda}\right]=\left(\frac{\operatorname{det} A(\lambda)}{p}\right)$ for any $\lambda \in \mathcal{Y}$, where

$$
A(\lambda):=\left(\begin{array}{cc}
\operatorname{tr}(\lambda) & \operatorname{tr}(\lambda e) \\
\operatorname{tr}(\lambda e) & \operatorname{tr}\left(\lambda e^{2}\right)
\end{array}\right) .
$$


Now we fix a non-square element $\sigma$ in $\mathbb{F}_{p^{2}}$. Then $\lambda$ satisfies (17) if and only if $\lambda \sigma$ does. This means that the multiplication by $\sigma$ leaves $\mathcal{Y}$ fixed. On the other hand, observe that $\left[M, W^{\lambda}\right]=-\left[M, W^{\lambda \sigma}\right]$ for any $\lambda \in \mathcal{Y}$. (Indeed,

$$
\operatorname{det} A(\lambda)=\sum_{i, j=0}^{n-1} \lambda^{p^{i}+p^{j}}\left(e^{2 p^{j}}-e^{p^{i}+p^{j}}\right) .
$$

Clearly, if $i-j$ is even, then $e^{2 p^{j}}=e^{p^{i}+p^{j}}$. If $i-j$ is odd, then $\sigma^{p^{i}+p^{j}}=\sigma^{p+1}$. This argument shows that $\operatorname{det} A(\lambda \sigma)=\sigma^{p+1} \operatorname{det} A(\lambda)$. Now $\sigma^{p+1}$ is a non-square in $\mathbb{F}_{p}$; hence the claim follows.) Consequently,

$$
\begin{gathered}
\sum_{\lambda \in \mathcal{Y}}\left(v(M), v\left(W^{\lambda}\right)\right)=\frac{1}{2} \sum_{\lambda \in \mathcal{Y}}\left(\left(v(M), v\left(W^{\lambda}\right)\right)+\left(v(M), v\left(W^{\lambda \sigma}\right)\right)\right) \\
=\frac{C_{0}}{2} \sum_{\lambda \in \mathcal{Y}}\left(\left[M, W^{\lambda}\right]+\left[M, W^{\lambda \sigma}\right]\right)=0 .
\end{gathered}
$$

2f) As a result of the computations in pp. 2c), 2d) and 2e), we obtain

$$
\begin{gathered}
p^{n / 2-1} C_{n-2}=\left(v(M), v\left(W^{\infty}\right)\right) \\
=\left(\sum_{\lambda \in \mathcal{X}}\left(v(M), v\left(W^{\lambda}\right)\right)+\sum_{\lambda \in \mathcal{Y}}\left(v(M), v\left(W^{\lambda}\right)\right)\right)=p^{n / 2-1} C_{0} C_{2},
\end{gathered}
$$

i.e. $C_{0}=C_{2} C_{n-2}$, as stated.

3) Finally, we prove (12) by induction on $n$. Because of the descent $n \sim n-1$ used in Theorem 4.4, we can restrict ourselves to the case $n$ is odd. The induction base $n=1,3$ has already been established, since we have proved (12) for $k=n, n-2$. For the induction step, observe that the descent $n \leadsto n-2$ used in the proof of Proposition 3.4 allows us to state that $C_{k}=\epsilon^{(n-k) / 2}$ for any odd $k \geq 3$. According to (14),

$$
C_{1}=C_{3} \cdot C_{n-2}=\epsilon^{(n-3) / 2} \cdot \epsilon=\epsilon^{(n-1) / 2},
$$

and the induction step is over.

Recall that $(v(L), v(M))$ is 0 if $n-\operatorname{dim}(L \cap M)$ is odd. Therefore, Theorem 5.3 completely determines the Gram matrix of the lattice $\Delta(p, n)$.

Corollary 5.4. Let $p$ be any odd prime and $n$ any odd integer. Relative to the generating system $\{v(L) \mid L \in \mathcal{L}(W)\}$ the unimodular lattice $\Delta(p, n)$ has the following Gram matrix:

$$
(v(L), v(M))=\left\{\begin{array}{cl}
\epsilon^{(n-k) / 2} p^{(k-1) / 2}[L, M], & \operatorname{dim}(L \cap M)=k \equiv 1 \bmod 2 \\
0, & \operatorname{dim}(L \cap M) \equiv 0 \bmod 2 .
\end{array}\right.
$$

Example 5.5. Let $p=n=3$. Then the Gram matrix for $\Delta(3,3)$ produced by Corollary 5.4 is the same as given in $[\mathrm{BaV}]$.

Corollary 5.6. Let $p$ be any odd prime and $n$ any even integer. Relative to the generating system $\{v(L) \mid L \in \mathcal{L}(W)\}$ the p-modular lattice $\Delta(p, n)$ has the following Gram matrix:

$$
(v(L), v(M))=\left\{\begin{array}{cl}
\epsilon^{(n-k) / 2} p^{k / 2}[L, M], & \operatorname{dim}(L \cap M)=k \equiv 0 \bmod 2, \\
0, & \operatorname{dim}(L \cap M) \equiv 1 \bmod 2 .
\end{array}\right.
$$


Example 5.7. Let $p$ be any odd integer. Then the $p$-modular $\left(p^{2}+1\right)$-dimensional lattice $\Delta(p, 2)$ is generated by $2(p+1)\left(p^{2}+1\right)$ vectors $v(L), L$ any oriented Lagrangian in $\mathbb{F}_{p}^{4}$. Here, $v(-L)=-v(L),(v(L), v(L))=p$, and $(v(L), v(M))$ equals $\epsilon[L, M]$ if $L \cap M=0$ and 0 if $\operatorname{dim}(L \cap M)=1$. Taking $p=5$, we get the 26dimensional 5 -modular lattice with minimum 5 constructed by Nebe.

Clearly, $(u(L), u(M))=p(v(L), v(M))$. Now we want to compute the scalar products $(u(L), v(M))$. Since $\sigma$ is determined up to sign, the scalar products $(u(L), v(M))$ are determined also up to sign. Recall that $(u(L), v(M))= \pm p^{[n / 2]}$ if $\operatorname{dim}(L \cap M)=n-1$ (cf. Lemma 3.6). For definiteness, we choose $\sigma$ such that

$$
(u(L), v(M))=p^{[n / 2]}[L, M]
$$

for oriented Lagrangians $L, M$ with $\operatorname{dim}(L \cap M)=n-1$.

Theorem 5.8. Let $p$ be any odd prime and $n$ any integer. Under the convention (18) one has

$$
(u(L), v(M))=(\epsilon / p)^{(n-1-k) / 2} p^{[n / 2]}[L, M]
$$

for any oriented Lagrangians $L, M$ with $k=\operatorname{dim}(L \cap M)$ and $n-k$ odd.

Proof. 1) By Lemma 3.6, Theorem 4.4 and Proposition 5.2, there are constants $D_{k}= \pm 1$ such that $[L, M](u(L), v(M))=p^{[n / 2]-(n-1-k) / 2} D_{k}$ for any oriented Lagrangians $L, M$ with $k=\operatorname{dim}(L \cap M)$ and $n-k$ odd. We want to show that

$$
D_{k}=\epsilon^{(n-1-k) / 2} \text {. }
$$

Clearly, (19) holds for $k=n-1$, due to (18). Because of the descent $n \leadsto n-1$ used in Theorem 4.4, it suffices to prove (19) for odd $n$.

2) Consider the standard spread of $W=\mathbb{F}_{p}^{2 n}$ (recall $n$ is odd). In the notation of p. 1) of the proof of Theorem 5.3 we set $L=W^{\infty}, M=\left(e_{1}, f_{2}, \ldots, f_{n}\right)$. Since $L \cap W^{\infty}$ has odd dimension, $\left(u(L), v\left(W^{\infty}\right)\right)=0$. Furthermore, for any $\lambda \in \mathbb{F}_{q}$, $L \cap W^{\lambda}=0$ and $\left[L, W^{\lambda}\right]=\epsilon^{n}$. Therefore, $\left(u(L), v\left(W^{\lambda}\right)\right)=\epsilon^{n} D_{0}$, yielding

$$
u(L)=\epsilon^{n} D_{0} p^{(1-n) / 2} \sum_{\lambda \in \mathbb{F}_{q}} v\left(W^{\lambda}\right) .
$$

On the other hand, by Theorem 5.3 we have

$$
v(M)=\epsilon^{(n-1) / 2} p^{(1-n) / 2} \sum_{\lambda \in \mathbb{F}_{q}, \operatorname{tr}\left(\lambda\left(\alpha_{1}\right)^{2}\right)=0} v\left(W^{\lambda}\right) .
$$

Hence $(u(L), v(M))=\epsilon^{(n+1) / 2} p^{(n-1) / 2} D_{0}$. But $\operatorname{dim}(L \cap M)=n-1$ and $[L, M]=\epsilon$; therefore we get $D_{0}=\epsilon^{(n-1) / 2}$. This establishes (19) for $k=0$.

Now we can prove (19) by induction on odd $n$. The induction base $n=1,3$ has already been established, since we have proved (19) for $k=n-1,0$. For the induction step, observe that the descent $n \leadsto n-2$ used in the proof of Proposition 3.4 allows us to state that $D_{k}=\epsilon^{(n-1-k) / 2}$ for any even $k \geq 2$, and so the induction step is over.

Next, let $p \equiv 1 \bmod 4$. We determine the Gram matrices for the lattices $\Delta^{-}(p, n)$. Recall (cf. $\left.\S 2\right)$ that $\Delta^{-}(p, n)$ has the same generating system as of $\Delta(p, n)$. But if $(\cdot, \cdot)$ is the scalar product on $\Delta(p, n)$, then $\Delta^{-}(p, n)$ is endowed with the scalar product $(\cdot, \cdot)^{-}$, where $(u, v)^{-}=p \mathbf{b}(u, v)+\mathbf{a}(\sigma u, v)$. Here $\mathbf{a}, \mathbf{b}$ are integers such that $\mathbf{a}^{2}-p \mathbf{b}^{2}=-1$. Also, here we have $\epsilon=1$. Bearing this in mind, from the above results we immediately obtain: 
Corollary 5.9. Let $p \equiv 1 \bmod 4$ be a prime and $n$ any even integer. Relative to the generating system $\{v(L) \mid L \in \mathcal{L}(W)\}$ the unimodular lattice $\Delta^{-}(p, n)$ has the following Gram matrix:

$$
(v(L), v(M))=\left\{\begin{array}{cc}
\mathbf{b} p^{k / 2}[L, M], & \operatorname{dim}(L \cap M)=k \equiv 0 \bmod 2, \\
\mathbf{a} p^{(k-1) / 2}[L, M], & \operatorname{dim}(L \cap M)=k \equiv 1 \bmod 2 .
\end{array}\right.
$$

Corollary 5.10. Let $p \equiv 1 \bmod 4$ be a prime and $n$ any odd integer. Relative to the generating system $\{v(L) \mid L \in \mathcal{L}(W)\}$ the p-modular lattice $\Delta^{-}(p, n)$ has the following Gram matrix:

$$
(v(L), v(M))=\left\{\begin{array}{cc}
\mathbf{b} p^{(k+1) / 2}[L, M], & \operatorname{dim}(L \cap M)=k \equiv 1 \bmod 2, \\
\mathbf{a} p^{k / 2}[L, M], & \operatorname{dim}(L \cap M)=k \equiv 0 \bmod 2 .
\end{array}\right.
$$

Example 5.11. Let $p \equiv 1 \bmod 4$. Then the $p$-modular $(p+1)$-dimensional lattice $\Delta^{-}(p, 1)$ has a basis consisting of the vectors $e_{\lambda}, \lambda \in \mathbb{F}_{p} \cup\{\infty\}$. These vectors are of norm $p \mathbf{b}$; furthermore, $\left(e_{\lambda}, e_{\infty}\right)=\mathbf{a}$ for any $\lambda \in \mathbb{F}_{p}$. Finally, for $\lambda \neq \mu \in \mathbb{F}_{p}$ we have $\left(e_{\lambda}, e_{\mu}\right)=\mathbf{a}$ if $\lambda-\mu$ is a square, and $-\mathbf{a}$ otherwise. Thus $\Delta^{-}(p, 1)$ is just the lattice $M_{p+1,2}$ constructed in Theorem (V.2) of [NPl].

Example 5.12. Let $p \equiv 1 \bmod 4$. Then the unimodular $\left(p^{2}+1\right)$-dimensional lattice $\Delta^{-}(p, 2)$ is generated by $2(p+1)\left(p^{2}+1\right)$ vectors $v(L), L$ any oriented Lagrangian in $\mathbb{F}_{p}^{4}$, with the following Gram matrix:

$$
(v(L), v(M))= \begin{cases}\mathbf{b} p[L, M], & \operatorname{dim}(L \cap M)=2, \\ \mathbf{a}[L, M], & \operatorname{dim}(L \cap M)=1, \\ \mathbf{b}[L, M], & \operatorname{dim}(L \cap M)=0 .\end{cases}
$$

Taking $p=5$ (and $\mathbf{a}=2, \mathbf{b}=1$ ), we get the 26-dimensional unimodular lattice with minimum 3 constructed by Nebe.

As we have mentioned above, the lattices $\Delta=\Delta(p, n)$ ( $n$ even), $\Delta^{-}(p, n)$ ( $p \equiv 1 \bmod 4$ and $n$ even) are $p$-modular. But they are not (self-dual) o-lattices (where $\mathfrak{o}=\langle 1,(1+\theta) / 2\rangle_{\mathbb{Z}}$ and $\theta=\sqrt{\epsilon p}$ ) by the following reason. The multiplication by $\theta$ should be given as $\theta(v)=\sigma(v), v \in \Delta$. If $\Delta$ is an o-lattice, then $\Delta$ contains $\frac{1+\theta}{2} v(L)=(v(L)+u(L)) / 2, L$ a Lagrangian. On the other hand, $((v(L)+u(L)) / 2, v(L))=p^{[n / 2]} / 2$ is not integral, a contradiction.

However, if we restrict ourselves to the $S_{n}$-stable lattices, then in some cases we can get self-dual o-lattices. Recall that an integral lattice $\Gamma$ is called a 2 -neighbour of a given integral lattice $\Lambda$ if the intersection $\Gamma \cap \Lambda$ has index 2 in both of $\Lambda$ and $\Gamma$.

Proposition 5.13. Let $\Delta$ denote any of the lattices $\Delta(p, n), \Delta^{-}(p, n)$. Then the following assertions hold.

(i) If $p \equiv 1 \bmod 4$, then $\Delta$ has no 2-neighbours.

(ii) Let $p \equiv 3 \bmod 4$. Then $\Delta$ has exactly two 2-neighbours, namely $\Delta^{\delta}=$ $\left\langle\Delta^{0}, \frac{1}{2}(v(L)+\delta u(L))\right\rangle_{\mathbb{Z}}$, where $\delta= \pm 1, \Delta^{0}$ the even part of $\Delta$ and $L$ a fixed Lagrangian. These neighbours are $S_{n}$-stable. If $p \equiv 7 \bmod 8$, then they are self-dual $\mathfrak{o}$-lattices (w.r.t. the Hermitian form $u \circ v$ defined in (3)) if $n$ is even, and even unimodular (Euclidean) $\mathfrak{o}$-stable lattices if $n$ is odd.

Proof. It is easy to see (cf. Lemma 6.5) that $\Delta^{0}$ is the unique sublattice of index 2 in $\Delta$. Hence, if $\Gamma$ is an arbitrary 2-neighbour of $\Delta$, then $\Gamma=\left\langle\Delta^{0}, w\right\rangle_{\mathbb{Z}}$ with $2 w \in \Delta$. By definition, $2 w \in \Delta \cap 2\left(\Delta^{0}\right)^{\#}=\Delta^{1}$ (see also the discussion before Lemma 6.5). 
Now $\Delta^{1} / 2 \Delta^{0}$ contains exactly 3 nontrivial cosets, namely those with representatives $v(L)$, and $w^{\delta}(L):=v(L)+\delta u(L), \delta= \pm 1$ and $L$ a fixed Lagrangian. The first coset is contained in $\Delta$; hence we can avoid it. Thus we can take $w=\frac{1}{2} w^{\delta}(L)$ and then $\Gamma=\Delta^{\delta}$. Observe that this $w$ has (squared) norm $p^{[n / 2]}(p+1) / 4$ if $\Delta=\Delta(p, n)$, $(\mathbf{b}(p+1)+2 \mathbf{a}) p^{(n+1) / 2}$ if $\Delta=\Delta^{-}(p, n)$ and $n$ is odd, and $(\mathbf{b}(p+1)+2 \mathbf{a}) p^{n / 2}$ if $\Delta=\Delta^{-}(p, n)$ and $n$ is even (cf. Theorems 5.3 and 5.8). Therefore, $\Gamma$ is integral (w.r.t. $(\cdot, \cdot))$ if and only if $p \equiv 3 \bmod 4$, and even if and only if $p \equiv 7 \bmod 8$.

Clearly, $G_{n}$ permutes the two cosets with representatives $w^{\delta}(L)$ in $\Delta^{1} / 2 \Delta^{0}$. But $S_{n}$ has no subgroups of index 2 ; hence $S_{n}$ stabilizes each of $\Delta^{\delta}$. On the other hand, $G_{n}$ permutes the lattices $\Delta^{\delta}$ transitively. (For recall that $u(L)=\sigma(v(L)$ ). Choose $g \in G_{n} \backslash S_{n}$ such that $g(L)=L$. Then $g\left(w^{\delta}(L)\right)=g(v(L))-\delta \sigma(g(v(L)))=$ $\pm w^{-\delta}(L)$.)

Observe that $\Delta^{0}, \Delta^{1}$ are always $\mathfrak{o}$-stable. Indeed, put $w^{\delta}(M)=v(M)+\delta u(M)$ for any oriented Lagrangian $M$. For any $s \in S_{n}$ with $s(L)=M$ one has $s\left(w^{\delta}(L)\right)=$ $\pm w^{\delta}(M)$. But we already know that $s$ fixes the coset $w^{\delta}(L)+2 \Delta^{0}$. Hence $w^{\delta}(L)+$ $w^{\delta}(M) \in 2 \Delta^{0}$. In other words, $(1+\sigma)(v(L)+v(M)) \in 2 \Delta^{0}$. This means $\Delta^{0}$ is $\mathfrak{o}$-stable, since we have $\theta v=\sigma(v)$ by definition and $\Delta^{0}$ is generated by the $v(L)+v(M)^{\prime}$ 's. Next, $\frac{1-\delta \sigma}{2}\left(w^{\delta}(L)\right)=\frac{1-\epsilon p}{2} v(L) \in \Delta^{1}$, as $\epsilon p \equiv 1 \bmod 4$. This implies that $\Delta^{1}$ is $\mathfrak{o}$-stable. This computation also convinces us that $\Delta^{\delta}$ is o-stable if and only if $p \equiv \pm 1 \bmod 8$.

Finally, assume $p \equiv 3 \bmod 4$. If $n$ is odd, then $\Delta$ is unimodular; hence $\Delta^{\delta}$ is unimodular, and even if $p \equiv 7 \bmod 8$. Suppose $n$ is even and $p \equiv 7 \bmod 8$. Then direct computation shows that $\Delta^{\delta} \circ \Delta^{\delta} \subseteq \mathfrak{o}$. On the other hand, $\Delta=\theta \Delta^{\#}=\Delta^{\perp}$ and $\Delta, \Delta^{\delta}$ are neighbours. Consequently, $\Delta^{\delta}$ is a self-dual o-lattice.

\section{Classification of invariant lattices}

The aim of this section is to prove Theorem 1.3. The case $p^{n}=3$ is trivial (see $[\mathrm{SchT}], \S 5)$, so throughout this section we suppose that $p^{n}>3$.

Let $H, \Gamma, \Delta$ be as in Theorem 1.3. Let $\rho$ denote the $H$-character afforded by $\Gamma$ and $\theta$ any irreducible constituent of $\rho$ restricted to $S:=S p_{2 n}(p)$. If $n \geq 2$, then the condition $1<\theta(1) \leq p^{n}+1$ implies by Theorem 5.2 [TZa 1] that $\theta \in$ $\{\psi, \bar{\psi}\}$; hence $\rho$ is absolutely irreducible. The same is true if $n=1$, except for the cases $p=3$ or $p \equiv 1 \bmod 6$, where $\left.\rho\right|_{S}$ can be irreducible. Thus, under the assumptions of Theorem 1.3, $\rho$ satisfies the assumptions of Lemma 2.1. Hence, $G:=H / K$ is as defined in Theorem 1.3, and $\rho$ is afforded by $\Delta$, i.e. $\rho=\chi$, $\Gamma \otimes \mathbb{C}=\Delta \otimes \mathbb{C}$. By the Deuring-Noether Theorem, the $\mathbb{Q} H$-modules $\Gamma \otimes \mathbb{Q}$ and $\Delta \otimes \mathbb{Q}$ are equivalent. Therefore, without loss of generality one may suppose that $\Gamma$ is a $G$-invariant sublattice in $\Delta$. Thus the proof of Theorem 1.3 reduces to the classification of $G$-invariant sublattices $\Gamma$ in $\Delta$.

For any Lagrangian $L$, let $S(L)$ be as defined in (5) and $R(L)=S \cap S(L)$. We start with the following observation.

Lemma 6.1. Let $n \geq 2$. Then the restriction of $\chi \bmod p$ to $R(L)$ contains the trivial character with multiplicity $\leq 4$.

Proof. Consider the standard embedding $T:=S L_{n}(p) \hookrightarrow R(L) \subseteq S p_{2 n}(p)$. It suffices to show that $1_{T}$ enters $\left.(\chi \bmod p)\right|_{T}$ with multiplicity at most 4 . Let $\theta$ denote the $S$-character of the Weil representation $\mathcal{W}$ (then $\theta$ is the sum of $\psi$ and another character of degree $\left.\left(p^{n}-1\right) / 2\right)$. If $n \geq 3$, then Zalesskii's formula for 
$\left.(\theta \bmod p)\right|_{T}$ [Zal] tells us that this character contains $1_{T}$ with multiplicity 2 . Since $\left.\chi\right|_{S}=\psi+\bar{\psi}$, we are done. Now let $n=2$. Then due to [Tiep 4], §3,

$$
\left.\theta\right|_{T}=2 \cdot 1_{T}+\xi_{1}+\xi_{2}+S t+2\left(\chi_{1}+\ldots+\chi_{(p-3) / 2}\right),
$$

where $\xi_{s}, S t, \chi_{s}$ are irreducible characters of $T$ of degree $(p+1) / 2, p$, and $p+1$, respectively. All the nontrivial characters occurring in this formula remain absolutely irreducible, being reduced modulo $p$. Hence $\left.(\theta \bmod p)\right|_{H}$ contains $1_{T}$ with multiplicity 2 , and so we are done.

Lemma 6.2. The module $V_{p}=\Delta / p \Delta$ has a unique nonzero proper $G$-submodule, and this submodule coincides with $\phi(\Delta) / p \Delta$.

Proof. 1) Let $A$ be any nonzero proper submodule in $V_{p}$. By Proposition 2.2, the Brauer character afforded by $A$ is $\eta_{i}$ for some $i=1,2$. In particular, $A$ is absolutely irreducible. An example of such a submodule $A$ is $\phi(\Delta) / p \Delta$. Therefore, the lemma is equivalent to saying that $V_{p}$ is indecomposable. Assume the contrary: $V_{p}$ is decomposable: $V_{p}=A \oplus B$. Clearly, $A$ and $B$ are isomorphic as $S$-modules. Hence, due to Lemma 2.5, in the case $p \equiv 1 \bmod 4$, it suffices to prove the lemma for one of the isoclinic groups $C_{2} \times G_{n}^{+}$and $G_{n}^{-}$. In what follows, we take $G=G_{n}^{-}$ if $n$ is odd, and $G=G_{n}^{+}$if $n$ is even; furthermore, $\Delta=\Delta(p, n)$.

If $n=1$, then due to [Ward 2], $A$ is a unique nonzero submodule of $V_{p}$ (and $A$ is called the modular quadratic residue code). This forces $V_{p}$ to be indecomposable, a contradiction. Therefore from now on we suppose that $n \geq 2$.

2) Let $L$ be any Lagrangian. By Proposition 3.1, the subgroup $S(L)$ fixes the vector $v(L)$. Observe that $v(L) \notin p \Delta$; hence one can view $v(L)$ as a nonzero vector in $V_{p}$. Set

$$
W(L)=\left\{v \in V_{p} \mid \forall \varphi \in S(L), \varphi(v)=v\right\} .
$$

Without loss of generality one may suppose that $\vartheta_{n} \in S(L)$, and so $S(L)=$ $\left\langle R(L), \vartheta_{n}\right\rangle$. For brevity, we denote by $\chi_{S}$ the restriction of $\chi \bmod p=\eta_{1}+\eta_{2}$ to $S(L)$, by $\chi_{R}$ the restriction of $\chi \bmod p$ to $R(L)$, by $\alpha$ the trivial character of $S(L)$, by $\beta$ the nontrivial character of degree 1 of $S(L)$ with $\operatorname{Ker} \beta=\left\langle R(L), \vartheta_{n}^{2}\right\rangle$. Write $v(L)=a+b$ for $a \in A, b \in B$. Remark that $a, b \neq 0$. (Assume the contrary: $a=0$. Then $v(L) \in B$ for any Lagrangian $L$. As $\Delta$ is generated by the vectors $v(M)$, which are acted on transitively by $S, B$ must be equal to the whole of $V_{p}$, a contradiction.) Now $S(L)$ fixes each of the subspaces $A, B$; therefore in fact $a, b \in W(L)$.

3) First consider the case $n$ is odd. Then $\eta_{i}$ is not self-dual by Proposition 2.2; hence $A$ and $B$ are totally singular relative to $(\cdot, \cdot)_{p}$, the reduction modulo $p$ of the scalar product. In particular, $(a, a)_{p}=(b, b)_{p}=0$. As $n \geq 3$, we have:

$$
0=(v(L), v(L))_{p}=(a+b, a+b)_{p}=(a, a)_{p}+(b, b)_{p}+2(a, b)_{p}=2(a, b)_{p},
$$

which implies that $(a, b)_{p}=0$. We have just shown that $C:=\langle a, b\rangle_{\mathbb{F}_{p}} \subseteq W(L)$ is totally singular with respect to $(\cdot, \cdot)_{p}: C \subseteq C^{\perp}$. Besides, the $S(L)$-modules $V_{p} / C^{\perp}$ and $C^{*}$ are isomorphic. (Recall that $\operatorname{det} \Delta=1$ in the case $n$ is odd.) From this it follows that $V_{p} / C^{\perp}$ affords the $S(L)$-character $2 \alpha$. Thus $\chi_{S}$ contains $\alpha$ with multiplicity at least 4 . In the proof of Proposition 3.1 we have singled out some subspace $U$ of $V$, which is acted on by $S(L)$ with character $\alpha+\beta$. From this it follows that $\chi_{S}$ contains $4 \alpha+\beta$, and so $\chi_{R}$ contains $1_{R(L)}$ with multiplicity at least 5 , contrary to Lemma 6.1 . 
4) Finally, let $n$ be even. Then both $\eta_{1}, \eta_{2}$ are of type + . Namely, the form $(\cdot, \cdot)_{p}$ is non-degenerate on $B$, since $A=p \Delta^{\#} / p \Delta$ is the radical of the form $(\cdot, \cdot)_{p}$. Also, $A$ carries the non-degenerate symmetric form $(x+p \Delta, y+p \Delta)_{p}^{\prime}=\frac{1}{p}(x, y) \bmod p$. Now $(b, b)_{p}=(v(L), v(L))_{p}=0$. Thus $C:=\langle b\rangle_{\mathbb{F}_{p}} \subseteq W(L)$ is totally singular with respect to $\left.(\cdot, \cdot)_{p}\right|_{B}: C \subseteq C^{\perp}$. Besides, the $S(L)$-modules $B / C^{\perp}$ and $C^{*}$ are isomorphic. From this it follows that $B / C^{\perp}$ and of course $\langle a\rangle_{\mathbb{F}_{p}}$ afford the $S(L)$ character $\alpha$. Thus $\chi_{S}$ contains $\alpha$ with multiplicity at least 3 .

On the other hand, $\chi_{S}$ contains $\beta$ with multiplicity at least 2 . (For set $D=$ $\langle u(L)\rangle_{\mathbb{F}_{p}}$. Since $(u(L), u(L))=p^{n / 2+1}, D$ is totally singular with respect to $(\cdot, \cdot)_{p}^{\prime}$ : $D \subseteq D^{\perp}$. Besides, the $S(L)$-modules $A / D^{\perp}$ and $D^{*}$ are isomorphic. From this it follows that $A / D^{\perp}$ affords the $S(L)$-character $\beta$.)

As a consequence, $\chi_{R}$ contains $1_{R(L)}$ with multiplicity at least 5 , contradicting Lemma 6.1.

Lemma 6.3. Let $r$ be a prime, $G$ a finite group, and $\Lambda$ an integral $G$-invariant lattice with the following properties:

(i) The $\mathbb{F}_{r} G$-module module $U=\Lambda / r \Lambda$ is uniserial, that is, it has a unique composition series $U=U_{0} \supset U_{1} \supset \ldots \supset U_{m}=0$;

(ii) Let $\Lambda_{i}$ be the inverse image of $U_{i}$ in $\Lambda, 0 \leq i \leq m-1$. Then the $\mathbb{F}_{r} G$-module $\Lambda_{i} / r \Lambda_{i}$ is also uniserial for any $i>0$.

Suppose that $\Gamma$ is any $G$-invariant sublattice in $\Lambda$ whose index is an $r$-power. Then $\Gamma$ is similar to one of the lattices $\Lambda_{i}, 0 \leq i \leq m-1$.

Proof. Denote $\Lambda_{m}=r \Lambda_{0}, \Lambda_{m+1}=r \Lambda_{1}$ and, more generally, $\Lambda_{k+m}=r \Lambda_{k}, k=$ $2,3, \ldots$ Our assumptions imply that

$$
\Lambda_{k} / r \Lambda_{k} \supset \Lambda_{k+1} / r \Lambda_{k} \supset \ldots \supset \Lambda_{k-1+m} / r \Lambda_{k} \supset 0
$$

is the unique composition series of the $\mathbb{F}_{r} G$-module $\Lambda_{k} / r \Lambda_{k}$. In particular, if $\Gamma$ lies between $\Lambda_{k}$ and $r \Lambda_{k}$, then $\Gamma=\Lambda_{k+j}$ for some $j, 0 \leq j \leq m$, and our claim follows.

Since $(\Lambda: \Gamma)$ is an $r$-power,

$$
\Lambda_{0}=\Lambda \supseteq \Gamma \supseteq r^{n} \Lambda=\Lambda_{n m}
$$

for some non-negative integer $n$. Let $\ell$ be the minimal non-negative integer such that $\Lambda_{i} \supseteq \Gamma \supseteq \Lambda_{i+\ell}$ for some $i$. We prove by induction on $\ell$ that $\Gamma$ is equal to some $\Lambda_{k}$. If $\ell \leq m$, we are done due to the above observation. Assume $\ell>m$. Without loss of generality we may suppose that $i=0$. Since $\Lambda_{0} \supseteq \Gamma+\Lambda_{\ell-m} \supseteq \Lambda_{\ell-m}$, by the induction hypothesis we get $\Gamma+\Lambda_{\ell-m}=\Lambda_{k}$ for some $k, 0 \leq k \leq \ell-m$. Now it is clear that $\Lambda_{k} \supseteq \Gamma \supseteq \Lambda_{\ell}$. By the minimality of $\ell$ we must have $k=0$, i.e., $\Gamma+\Lambda_{\ell-m}=\Lambda_{0}$. This implies

$$
\Lambda_{0} \supseteq \Gamma \supseteq p\left(\Gamma+\Lambda_{\ell-m}\right)=p \Lambda_{0}=\Lambda_{m},
$$

contrary to the choice of $\ell$. The induction step is over.

Corollary 6.4. If $\Gamma$ is any $G_{n}$-invariant sublattice of $\Delta$ with the index $(\Delta: \Gamma)$ being a power of $p$, then there exists an integer $k \geq 0$ such that $\Gamma=\phi^{k}(\Delta)$.

Proof. By Lemma $6.2, \phi(\Delta) / p \Delta$ is the unique nonzero proper submodule of $V_{p}$; hence $V_{p}$ is uniserial. Suppose that $\phi(\Delta) \supset \Lambda \supset p \phi(\Delta)$ for some $G_{n}$-invariant sublattice $\Lambda$. Since $g \phi g^{-1}= \pm \phi$ for all $g \in G_{n}, \phi^{-1}(\Lambda)$ is a $G_{n}$-stable sublattice lying between $\Delta$ and $p \Delta$, which implies that $\phi^{-1}(\Lambda)=\phi(\Delta), \Lambda=p \Delta$. Thus the module $\phi(\Delta) / p \phi(\Delta)$ is also uniserial. Now we can apply Lemma 6.3. 
Since $\Delta$ is an odd lattice, the even part $\Delta^{0}$ is a $G$-invariant sublattice of index 2 containing $2 \Delta$. Also, $\Delta^{1}=\Delta \cap 2\left(\Delta^{0}\right)^{\#}$ is another $G$-invariant sublattice containing $2 \Delta$.

Lemma 6.5. The $\mathbb{F}_{2} G$-module $V_{2}=\Delta / 2 \Delta$ has precisely two nontrivial proper submodules, namely, $\Delta^{i} / 2 \Delta$ with $i=0,1$. Moreover, if $\Gamma$ is any $G$-invariant sublattice of $\Delta$ with the index $(\Delta: \Gamma)$ being a power of two, then there exists an integer $k \geq 0$ such that

$$
\Gamma \in\left\{2^{k} \Delta, 2^{k} \Delta^{0}, 2^{k} \Delta^{1}\right\} .
$$

Proof. Observe that $\mathbf{a}$ is even and $\mathbf{b}$ is odd. Hence the lattices $\Delta(p, n)$ and $\Delta^{-}(p, n)$ have the same Gram matrix modulo 2. In particular, in calculating scalar products modulo 2 we can restrict ourselves to $\Delta(p, n)$.

1) At this point we show that $S:=S p_{2 n}(p)$ fixes a unique nonzero vector $w$ in $V_{2}$, and $w=v(L)+u(L)$ for any Lagrangian $L$. To this end, we first observe that det $\Delta$ is odd; hence the reduction $(\cdot, \cdot)_{2}$ of the scalar product is non-degenerate on $V_{2}$. Next, putting $w(L)=v(L)+u(L)$, by Theorems 5.3 and 5.8 we see that $(w(L), v(M))_{2}=1$ for any arbitrary Lagrangian $M$. If $\varphi \in G$ and $\varphi(L)=L^{\prime}$, then $\varphi(w(L))=w\left(L^{\prime}\right)\left(\right.$ in $\left.V_{2}\right)$. Hence $(\varphi(w(L))-w(L), v(M))_{2}=0$. But $V_{2}$ is generated by the vectors $v(M)$ and $(\cdot, \cdot)_{2}$ is non-degenerate. Therefore, $\varphi(w(L))=w(L)$. Thus $w:=w(L)$ is $G$-stable. Conversely, let $w^{\prime} \in V_{2}$ be a nonzero vector which is fixed by $S$. Since $S$ acts transitively on the vectors $v(M), M$ any Lagrangian, there exists $\lambda \in \mathbb{F}_{2}$ such that $\left(w^{\prime}, v(M)\right)_{2}=\lambda$ for all $M$. If $\lambda=0$, then the nondegeneracy of $(\cdot, \cdot)_{2}$ implies that $w^{\prime}=0$, contrary to the choice of $w^{\prime}$. If $\lambda=1$, then $\left(w-w^{\prime}, v(M)\right)_{2}=0$, yielding $w^{\prime}=w$.

2) Set $U_{0}=\Delta^{0} / 2 \Delta, U_{1}=\langle w\rangle_{\mathbb{F}_{2}}$. Clearly, $\Delta^{0}$ and so $U_{0}$ are generated by the vectors of the form $v(L)+v(M), L, M$ any Lagrangians. Since $(w, v(L)+v(M))_{2}=0$, we see that $U_{1}=\Delta^{1} / 2 \Delta$. Also, $w \in U_{0}$. By Proposition 2.2 (ii), $0 \subset U_{1} \subset U_{0} \subset V_{2}$ is a composition series of the $\mathbb{F}_{2} G$-module $V_{2}$, with two trivial composition factors and one (absolutely) irreducible factor of dimension $p^{n}-1$. Clearly, $U_{0}$ and $U_{1}$ are dual to each other w.r.t. $(\cdot, \cdot)_{2}$.

Now let $U$ be any nonzero proper $G$-submodule in $V_{2}$. Then $\operatorname{dim} U \in\left\{1,2, p^{n}-\right.$ $\left.1, p^{n}\right\}$. If $\operatorname{dim} U=1$, then $U$ must be generated by a nonzero $G$-stable vector; hence $U=U_{1}$ due to 1 ). If $\operatorname{dim} U=p^{n}$, then the dual module $U^{\perp}$ has dimension 1 ; therefore $U^{\perp}=U_{1}$, which implies that $U=U_{0}$. Assume $\operatorname{dim} U=2$. Then the action of $S$ on $U$ induces a homomorphism from $S$ to $G L(U)=G L_{2}(2) \simeq \mathbb{S}_{3}$. But $S=S p_{2 n}(p)$ is perfect (as $p^{n}>3$ ); therefore this homomorphism is trivial, i.e. $S$ acts trivially on $U$. In this case, $V_{2}$ has at least three (distinct) $S$-stable vectors, contrary to 1 ). If $\operatorname{dim} U=p^{n}-1$, then the dual module $U^{\perp}$ has dimension 2, again a contradiction.

We have shown that $V_{2}$ has just two nontrivial proper submodules: $U_{0}$ and $U_{1}$.

3) Next we consider any nontrivial proper submodule $U$ in $V_{4}=\Delta / 4 \Delta$, and suppose that $U \nsubseteq 2 V_{4}$. Then $\left(U+2 V_{4}\right) / 2 V_{4}$ is a nonzero submodule in $V_{4} / 2 V_{4} \simeq V_{2}$. By the results of 2$),\left(U+2 V_{4}\right) / 2 V_{4}$ contains $U_{1}$. From this it follows that $U$ contains a vector $w^{\prime}=w+2 x$ for a certain $x \in V_{4}$. Pick an element $\varphi \in G(L)$ such that $\varphi: v(L) \mapsto v(L), u(L) \mapsto-u(L)$. Then $w^{\prime}+\varphi\left(w^{\prime}\right)=2 y$, where $y=v(L)+x+\varphi(x)$. Since $x+\varphi(x) \in U_{0}=\langle w\rangle^{\perp}$, we get $(w, y)_{2}=(w, v(L))_{2}=1$, which means that $y \notin U_{0}$. We have seen that $U^{\prime}=\left(U \cap 2 V_{4}\right) / 4 V_{4}$ is a $G$-submodule in $2 V_{4} / 4 V_{4} \simeq 2 V_{2}$, which contains a vector $2 y \notin 2 U_{0}$. By the results of 2$), U^{\prime}=2 V_{4} / 4 V_{4}$, i.e. $U \supseteq 2 V_{4}$. This means: if $U$ is any $G$-submodule of $V_{4}$, then either $U \supseteq 2 V_{4}$, or $U \subseteq 2 V_{4}$. 
4) Finally, let $\Gamma$ be any $G$-invariant sublattice of $\Delta$ with $(\Delta: \Gamma)=2^{m}$. Then $\Delta \supseteq \Gamma \supseteq 2^{m} \Delta$. We prove by induction on $m \geq 0$ that there exists an integer $k \geq 0$ such that $\Gamma \in\left\{2^{k} \Delta, 2^{k} \Delta^{0}, 2^{k} \Delta^{1}\right\}$. This claim is obvious if $m=0$ or 1 (see item $2)$ ). Now assume $m \geq 2$. Then $\Delta \supseteq \Gamma+4 \Delta \supseteq 4 \Delta$. Due to 3), either $\Gamma+4 \Delta \subseteq 2 \Delta$, or $\Gamma+4 \Delta \supseteq 2 \Delta$. In the former case, $2 \Delta \supseteq \Gamma \supseteq 2^{m} \Delta$; therefore $\Delta \supseteq \frac{1}{2} \Gamma \supseteq 2^{m-1} \Delta$, and one can now use the induction hypothesis. In the latter case,

$$
2^{m-1} \Delta \subseteq 2^{m-2}(\Gamma+4 \Delta)=2^{m-2} \Gamma+2^{m} \Delta \subseteq \Gamma
$$

and one can again use the induction hypothesis.

Proof of Theorem 1.3. Consider any $G$-invariant lattice $\Gamma$ lying in $\Delta$. We may suppose that $\Gamma \nsubseteq k \Delta$ for any integer $k>1$. Clearly, $\Gamma \supseteq l \Delta$ for some natural $l$. Choose minimal natural $\ell$ with the property $\Gamma \supseteq \ell \Delta$. If $\ell=1$, then $\Gamma=\Delta$. Assume that $\ell>1$. Claim that $\ell=2^{a} p^{b}$ for some non-negative integers $a, b$. (For assume the contrary: $\ell$ is divisible by an odd prime $r, r \neq p$. Observe that $(\Gamma+r \Delta) / r \Delta$ is a nonzero $G$-module in $V_{r}=\Delta / r \Delta$. By Proposition 2.2 (i), $(\Gamma+r \Delta) / r \Delta=V_{r}$, $\Gamma+r \Delta=\Delta$. Hence,

$$
\frac{\ell}{r} \Delta=\frac{\ell}{r}(\Gamma+r \Delta)=\frac{\ell}{r} \Gamma+\ell \Delta \subseteq \Gamma,
$$

contradicting the minimality of $\ell$.)

Setting $\widetilde{\Gamma}=\Gamma+p^{b} \Delta$, one has

$$
\Delta \supset \widetilde{\Gamma} \supseteq p^{b} \Delta, \widetilde{\Gamma} \supseteq \Gamma \supseteq 2^{a} \Gamma+\ell \Delta=2^{a} \widetilde{\Gamma} .
$$

By Corollary 6.4, $\widetilde{\Gamma}=\phi^{k}(\Delta)$. Replacing $\Gamma$ by $\phi^{-k}(\Gamma)$, which is isometrically similar to $\Gamma$, we can suppose that $k=0$, i.e. $\widetilde{\Gamma}=\Delta$. In this case, $\Delta \supset \Gamma \supseteq 2^{a} \Delta$. By Lemma $6.5, \Gamma$ is similar to one of the lattices $\Delta, \Delta^{0}, \Delta^{1}$.

\section{Properties of $\Delta(p, n)$}

This section is very sketchy, because a detailed exposition has been given in [SchT], $\S \S 4,6$. It turns out that the arguments, given there for the case $p^{n} \equiv$ $3 \bmod 4$, are also applicable to the case $p^{n} \equiv 1 \bmod 4$. Hence we restrict ourselves to exposing the results, which hold for any odd prime $p$, but omitting the proofs.

For short we denote $G=G_{n}^{-}$if $n$ is odd, and $G=G_{n}^{+}$if $n$ is even. Furthermore, $p$ is any odd prime and $\Delta=\Delta(p, n)$.

First we consider the $G$-invariant odd unimodular lattice $\Delta=\Delta(p, 3)$ obtained in Theorem 3.9. The generating vectors $v(L)$ now have norm $(v(L), v(L))=p$, and $\Delta$ contains a $p$-scaled unit lattice $\Gamma$, spanned by $N:=p^{3}+1$ pairwise orthogonal vectors of norm $p$ (for instance, the $v(L)$, where $L$ runs over a symplectic spread). Therefore, $\Delta$ can be described (non-canonically) by a subspace $C:=\Delta / \Gamma \subset \Gamma^{\#} / \Gamma=\frac{1}{p} \Gamma / \Gamma \simeq \mathbb{F}_{p}^{N}$, that is, by a linear code over $\mathbb{F}_{p}$. In this way we obtain an injective mapping $\pi \mapsto C=C(\pi)$ from the set $\mathcal{S}$ of all isomorphism classes of symplectic spreads $\pi$ of $W=\mathbb{F}_{p}^{6}$ to the set $\mathcal{C}$ of all equivalence classes of self-dual codes $C$ of length $p^{3}+1$ over $\mathbb{F}_{p}$. Moreover, $\operatorname{Aut}(C(\pi))=\operatorname{Aut}(\pi) / C_{(p-1) / 2}$. Observe that the definition of $\operatorname{Aut}(\pi)$ used in this paper differs from the one given in [SchT]. In particular, the central subgroup $C_{(p-1) / 2}$ of $\operatorname{Aut}(\pi)$ acts trivially on every vector $v(L)$, hence on $C(\pi)$.

Now we turn to the case $n \geq 5$ and $n$ is odd. Let

$$
\pi=\left\{W_{i} \mid 1 \leq i \leq p^{n}+1\right\}
$$


be a symplectic spread of $W=\mathbb{F}_{p}^{2 n}$. Set

$$
v_{i}=v\left(W_{i}\right), \quad \Gamma=\Delta(\pi)=\left\langle v_{i} \mid 1 \leq i \leq p^{n}+1\right\rangle_{\mathbb{Z}} .
$$

For brevity we denote $\ell=(n-1) / 2$. Then

$$
\Gamma \subset \Delta=\Delta^{\#} \subset \Gamma^{\#}=p^{-\ell} \Gamma \text {. }
$$

For each $j, 1 \leq j \leq \ell+1$, one can view $H_{j}=p^{j-1} \Gamma^{\#} / p^{j} \Gamma^{\#}$ as standard orthogonal space over $\mathbb{F}_{p}$, with the basis $\left(p^{j-1-\ell} v_{i} \mid 1 \leq i \leq p^{n}+1\right)$ and with the form $(x, y)_{(j)}=p^{\ell+2-2 j}(x, y) \bmod p$. (Here and below, we identify the coset $x+p^{j} \Gamma^{\#}$ with $x$.) Clearly, the $H_{j}$ 's are isometric to each other, and so one can identify them canonically with $H=H_{\ell+1}$. Keeping this identification in mind, we can view every factor-group

$$
C_{j}=\left(\left(\Delta \cap p^{j-1} \Gamma^{\#}\right)+p^{j} \Gamma^{\#}\right) / p^{j} \Gamma^{\#}
$$

as a linear code of length $p^{n}+1$ over $\mathbb{F}_{p}$, with the ambient space $H$. It is obvious that $C_{1} \subseteq C_{2} \subseteq \ldots \subseteq C_{\ell}$. One shows that $C_{j}^{\perp}=C_{\ell+1-j}$ for $1 \leq j \leq \ell$. In particular, $C_{j}$ is self-orthogonal if $1 \leq j \leq(\ell+1) / 2$; and $C_{(n+1) / 4}$ is self-dual if $n \equiv 3 \bmod 4$.

Now we take $\pi$ to be the standard symplectic spread $\pi_{D}$. Then the same arguments as in the proof of Proposition 4.6 [SchT] assure that all the codes $C_{j}$, $1 \leq j \leq \ell$, are among the $G L_{2}(q)$-codes having a $\mathbb{F}_{p}$-form, which have been introduced by Ward in [Ward 2]. (Actually, Ward uses an irreducible representation of $H=G L_{2}(q)$ with kernel $T=C_{(q-1) / 2}$, where $q=p^{n}$. But $H / T \simeq R / K$, where $R=S L_{2}(q) \cdot C_{p-1}$ and $K=R \cap T \simeq C_{(p-1) / 2}$, cf. page 1 of the proof of Proposition 2.3. Now Ward's representation coincides with the action of $R / K$ on $\Delta$.) He has shown that the lattice of his $G L_{2}(q)$-codes is inversely isomorphic to the lattice of the so-called closed subsets of $\mathbb{F}_{2}^{n}$. He has also distinguished the following analogues of Reed-Muller codes. View elements of $\mathbb{F}_{2}^{n}$ as binary words of length $n$ and take $B_{w}$ to be the set of all binary words of length $n$ and weight $\leq w$. Then $B_{w}$ is closed and cyclic (in the sense of [Ward 2]), and Ward's correspondence gives us a $G L_{2}(q)$-code $\mathcal{C}_{n, w}$ over $\mathbb{F}_{p}, 0 \leq w \leq n-1$. The middle code is just $\mathcal{C}_{n,(n-1) / 2}$; more generally, $\mathcal{C}_{n, w}^{\perp}=\mathcal{C}_{n, n-1-w}$. We conjecture that the above codes $C_{j}$ are equal to $\mathcal{C}_{n, n-2 j}$ for $j, 1 \leq j \leq \ell=(n-1) / 2$. Without this conjecture, we can only give the following lower bound for the minimum of $\Delta$ which is unfortunately independent of $n$. A proof of the conjecture would lead to a lower bound $\left(p^{[n / 2]}+1\right) / 2$ instead of $(p+1) / 2$.

Proposition 7.1. Let $p$ be any odd prime and $n \geq 2$ arbitrary. Then

$$
\max \left\{3, \frac{p+1}{2}\right\} \leq \min \Delta(p, n) \leq p^{[n / 2]} .
$$

For the proof, observe that $\Delta(p, n)$ with even $n$ is a sublattice of $\Delta(p, n+1)$; hence it suffices to prove Theorem 7.1 for odd $n$. The inequality $\min \Delta(p, n) \geq 3$ has been mentioned in Theorem 1.1. Now one repeats the proof of Proposition 6.4 [SchT].

Remark 7.2. Observe that the lattices $\Delta(p, n), n>1$ odd, are unimodular lattices with relatively short shadow. More precisely, recall that a characteristic vector of a unimodular lattice $\Lambda$ is any vector $w \in \Lambda$ such that $(v, w) \equiv(v, v) \bmod 2$ for all 
$v \in \Lambda$, and the coset $\frac{1}{2} w+\Lambda$ is called the shadow of $\Lambda$ in [CoS 2]. It is known that $(w, w) \equiv \operatorname{rank} \Lambda \bmod 8$ for any characteristic vector $w$. Define

$$
e(\Lambda)=\frac{1}{8}(\operatorname{rank} \Lambda-\min \{w \mid w \text { any characteristic vector of } \Lambda\}) .
$$

Clearly, $e(\Lambda)=\frac{1}{8} \operatorname{rank} \Lambda$ if and only if $\Lambda$ is an even (unimodular) lattice. Elkies [Elk] has shown that $\Lambda=\mathbb{Z}^{m}$ is the unique unimodular lattice with $e(\Lambda)=0$; all other lattices have $e \geq 1$. Moreover, he has described all the unimodular lattices $\Lambda$ with $e(\Lambda)=1$.

Clearly, $e(\Delta(p, 1))=0$. We observe that $e(\Delta)=2$, if $\Delta=\Delta(3,3)$ or $\Delta^{-}(5,2)$. More generally, we claim that

$$
\frac{1}{8}\left(p^{k}-1\right)\left(p^{k+1}-1\right) \leq e(\Delta) \leq \frac{1}{8} p^{k+1}\left(p^{k}-1\right)
$$

if $\Delta:=\Delta(p, 2 k+1)$, which means in particular that $\Delta$ has a relatively short shadow. (For, from Theorems 5.3 and 5.8, it follows that $v(L)+u(L)$ is a characteristic vector of norm $p^{k}(p+1), L$ any Lagrangian. On the other hand, if $w$ is any characteristic vector, then $(w, v(M)) \equiv 1 \bmod 2$ for any Lagrangian $M$. Hence, if $\pi$ is a symplectic spread in $\mathbb{F}_{p}^{2 k+1}$ and $w=\sum_{M \in \pi} a_{M} v(M)$, then $a_{M} \neq 0$. But $p^{k} a_{M}=(v(M), w) \in$ $\mathbb{Z}$; hence $a_{M} \geq p^{-k}$. As a consequence, $(w, w) \geq p^{-2 k} \sum_{M \in \pi}(v(M), v(M))$, and so $(w, w) \geq p^{k+1}+1$.) Specializing $p=3$ and $k=1$, one gets $e(\Delta(3,3))=2$. Next, let $p=5$ and $k=2$. Then again $v(L)-u(L)$ is a characteristic vector, of norm 10, yielding $e(\Delta) \geq 2$ for $\Delta=\Delta^{-}(5,2)$. On the other hand, if $e(\Delta)>2$, then $e(\Delta)=3$ and $\Delta$ would have a (characteristic) vector of norm $26-3 \cdot 8=2$, contrary to the fact that $\min \Delta=3$.

\section{REFERENCES}

$[\mathrm{BaV}] \quad$ R. Bacher and B. Venkov, Lattices and association schemes: A unimodular example without roots in dimension 28, Annales Inst. Fourier 45 (1995), 1163 - 1176. MR 96j:11093

[BRW] B. Bolt, T. G. Room and G. E. Wall, On the Clifford collineation, transform and similarity groups. I, J. Austral. Math. Soc. 2 (1961-62), 60 - 79. MR 23:A3171

[BuS] P. Buser and P. Sarnak, On the period matrix of a Riemann surface of large genus (with an Appendix by J. H. Conway and N. J. A. Sloane), Invent. Math. 117 (1994), $27-56$. MR 95i: 22018

[Coh] H. Cohn, "A Classical Invitation to Algebraic Numbers and Class Fields", SpringerVerlag, 1978. MR 80c: 12001

[Atlas] J.H. Conway et al., "An ATLAS of Finite Groups", Oxford University Press, 1985. MR 88g:20025

[CoS 1] J. H. Conway and N. J. A. Sloane, On lattices equivalent to their duals, J. Number Theory 48 (1994), 373 - 382. MR 95j: 11063

[CoS 2] J. H. Conway and N. J. A. Sloane, A new upper bound on the minimal distance of self-dual codes, IEEE Trans. Inform. Theory 36 (1990), 1319-1333. MR 91m:94024

[CoT] A. M. Cohen and Pham Huu Tiep, Splitting fields for Jordan subgroups, in: 'Finite Reductive Groups, Related Structures and Representations', ed. by M. Cabanes, Progress in Math., vol. 141, 1996, Birkhäuser, pp. 165 - 183. MR 98a:20044

[DiM] F. Digne and J. Michel, "Representations of Finite Groups of Lie Type", London Math. Soc. Student Texts 21, Cambridge Univ. Press, 1991, 159 pp. MR 92g:20063

[Dum] N. Dummigan, Symplectic group lattices as Mordell-Weil sublattices, J. Number Theory 61 (1996), $365-387$. MR 98h:11088

[Elk] N. Elkies, A characterization of the $\mathbf{Z}^{n}$ lattice, Math. Res. Letters 2 (1995), $321-326$. MR 96h:11064

[Gow] R. Gow, Even unimodular lattices associated with the Weil representation of the finite symplectic group, J. Algebra 122 (1989), 510 - 519. MR 90f:20009 
[GoW] R. Gow and W. Willems, On the geometry of some simple modules in characteristic 2, J. Algebra 195 (1997), 634-649.

[Gro] B. H. Gross, Group representations and lattices, J. Amer. Math. Soc. 3 (1990), $929-$ 960. MR 92a:11077

[Isa] I. M. Isaacs, Characters of solvable and symplectic groups, Amer. J. Math. 95 (1973), 594 - 635. MR 48:11270

[KoT] A. I. Kostrikin and Pham Huu Tiep, "Orthogonal Decompositions and Integral Lattices", Walter de Gruyter, Berlin et al, 1994, 535 pp. MR 96f:17001

[LiV] G. Lion and M. Vergne, "The Weil Representation, Maslov Index and Theta Series", Progress in Math. v. 6, Birkhäuser, Boston, Mass., 1980, 337 pp. MR 81j:58075

[NPl] G. Nebe and W. Plesken, "Finite Rational Matrix Groups", Mem. Amer. Math. Soc. 116 (1995), no. 556, 144 pp. MR 95k:20081

[Que] H.-G. Quebbemann, Modular lattices in Euclidean spaces, J. Number Theory 54 (1995), 190 - 202. MR 96i:11072

[SchHem] R. Scharlau, B. Hemkemeier, Classification of integral lattices with large class number, Math. Comp. 67 (1998), 737-749. MR 98g:11042

[SchT] R. Scharlau and Pham Huu Tiep, Symplectic groups, symplectic spreads, codes and unimodular lattices, J. Algebra 194 (1997), 113-156. MR 98g:20075

[Sei] G. M. Seitz, Some representations of classical groups, J. London Math. Soc. (2) 10 (1975), 115 - 120. MR 51:5789

[Tiep 1] Pham Huu Tiep, Weil representations of finite symplectic groups, and Gow lattices, Mat. Sb. 182 (1991), no. 8, 1161-1183; English transl. in Math. USSR-Sb. 73 (1992), no. $2,535-555$.

[Tiep 2] Pham Huu Tiep, Globally irreducible representations of finite groups and integral lattices, Geometriae Dedicata 64 (1997), 85 - 123. MR 98e:20011

[Tiep 3] Pham Huu Tiep, Globally irreducible representations of the finite symplectic group $\mathrm{Sp}_{4}(q)$, Commun. Algebra 22 (1994), 6439 - 6457.

[Tiep 4] Pham Huu Tiep, Weil representations as globally irreducible representations, Math. Nachr. 184 (1997), 313 - 327. MR 98b:20010

[TZa 1] Pham Huu Tiep and A. E. Zalesskii, Minimal characters of the finite classical groups, Commun. Algebra 24 (1996), 2093 - 2167. MR 97f:20018

[TZa 2] Pham Huu Tiep and A. E. Zalesskii, Some characterizations of the Weil representations of the symplectic and unitary groups, J. Algebra 192 (1997), 130-165. CMP 97:13

[Ward 1] H. N. Ward, Representations of symplectic groups, J. Algebra 20 (1972), $182-195$. MR 44:4116

[Ward 2] H. N. Ward, Quadratic residue codes in their prime, J. Algebra 150 (1992), 87 - 100. MR 94a:11194

[Zal] A. E. Zalesskii, Decompositions numbers modulo $p$ of certain representations of the groups $S L_{n}\left(p^{k}\right), S U_{n}\left(p^{k}\right), S p_{2 n}\left(p^{k}\right)$, in: 'Topics in Algebra', Banach Center Publications, vol. 26, pt. 2, Warsaw 1990, pp. 491 - 500. MR 93e:20022

Department of Mathematics, University of Dortmund, 44221 Dortmund, Germany

E-mail address: rudolf.scharlau@mathematik.uni-dortmund.de

Department of Mathematics, Ohio State University, Columbus, Ohio 43210

Current address: Department of Mathematics, University of Florida, Gainseville, Florida 32611

E-mail address: tiep@math.ufl.edu 